\title{
A REVISION OF ZAEUCOILA ASHMEAD (HYMENOPTERA, FIGITIDAE), PARASITOIDS OF AGROMYZIDAE (DIPTERA): NEW SPECIES, IDENTITY, DISTRIBUTION, AND HOSTS
}

urn:1sid:zoobank.org:pub:65DC83D0-4192-4379-B37D-D5CD205115BE

Matthew L. Buffington, Fabiana E. Gallardo, Vanina Reche, and Mattias Forshage

(MLB) Systematic Entomology Laboratory, ARS-USDA, c/o National Museum of Natural History, Smithsonian Institution, 10th \& Constitution Ave NW, MRC 168, Washington, DC 20560, USA (e-mail: matthew.buffington@ars.usda.gov); (FEG and VR) División Entomología, Museo de La Plata, Facultad de Ciencias Naturales y Museo, Universidad Nacional de La Plata. Paseo del Bosque, 1900 La Plata, Argentina. Comisión de Invvestigaciones Científicas de la provincia de Buenos Aires (CIC); (MF) Swedish Museum of Natural History, Box 50007, 10405 Stockholm, Sweden

(MLB) urn:1sid:zoobank.org:author:603275DE-9AE3-40C6-8AD7-6A2AF7485F35 (FEG) urn:1sid:zoobank.org:author:B92F9D21-9A41-4B56-A6BE-67DFB1523DA5 (VR) urn:1sid:zoobank.org:author:E2D6D1BE-EA84-440D-A28A-6794576FFF50 (MF) urn:1sid:zoobank.org:author:F26F07CF-D637-4897-8EE4-D7BC805A196B

Abstract.-The eucoiline genus Zaeucoila Ashmead is revised. Following a reevaluation combining previously published phylogenetic data and new specimens, Agrostocynips Díaz is synonymized with Zaeucoila. All species included in Zaeucoila are systematically treated, and now amounts to twelve, seven of which are described as new here: Zaeucoila bitiburculata new species, Z. fidalgoii new species, Z. infuscata new species, Z. johnsonii new species, Z. lignys new species, Z. normae new species, Z. patera new species. The following new combinations are proposed and species redescribed: Zaeucoila grenadensis (Ashmead), new combination; Z. robusta (Ashmead), new combination; Z. flavipes (Ashmead), new combination. Agrostocynips diastrophi (Ashmead), Agrostocynips clavatus Díaz, and Agrostocynips enneatoma (Díaz) are new synonyms of Zaeucoila robusta. Zaeucoila incompleta (Kieffer), Z. triangulifera Kieffer, and Z. unicarinata Ashmead are redescribed. Zaeucoila is an unusual genus of Zaeucoilini in its wide distribution area: it can be found from southern Argentina well into the eastern United States and southern Canada; no other zaeucoiline genus shows such a pattern. Host records and geographic data for Zaeucoila are brought up to date, as well as a key to species. Zeucoila are primary parasitoids of Agromyzidae, including Liriomyza trifolii and other species that are notable economic pests of agriculture. Tropideucoila fulvonotata (Kieffer) is transferred to Marthiella (new combination).

Key Words: Cynipoidea, new species, distribution records, species key, Agroymizidae, parasitoid, redescription, new species 
Zaeucoila Ashmead, 1903, (Hymenoptera: Figitidae: Eucoilinae) was described based on specimens (from Brazil) sent to William Ashmead of the US National Museum (National Museum of Natural History, Smithsonian Institution). Jean Jacques Kieffer (1907) later added two more species collected by C. F. Baker in the Caribbean. The genus was not actively researched over the following five decades, but was included in the keys of Weld (1952). Focus was renewed on Zaeucoila s.l. through the work of Norma Díaz of La Plata, Argentina, in the 1970's. During this time, research was conducted on natural enemies of agromyzid flies of economic concern, and through this research, Agrostocynips Díaz was described, containing A. clavatus Díaz (Díaz 1975, 1976) and later A. enneatoma (Díaz) (Gallardo and Díaz 1997). These species looked superficially like Zaeucoila, but differed in a number of characters (e.g., genal carina absent; mesoscutum lacking a keel) that seemed to justify the circumscription of a new genus.

Through Nordlander's ground-breaking research on eucoilines in the late 1970s and early 1980s, it was determined that Zaeucoila, along with several other lineages of eucoilines associated with agromyzids, formed what appeared to be a natural group of genera, and was so named the Gronotoma-group of genera (Nordlander 1982). Díaz and Gallardo (1997) disagreed with this original treatment, and instead placed three of the Neotropical genera included originally in the Gronotoma-group into a new group called the Zaeucoila-group (these genera being Rhabdeucoela Kieffer, Penteucoila Weld and Zaeucoila); to this group, over subsequent years, they added Agrostocynips
Díaz, Tropideucoila Ashmead, Lopheucoila Weld, Dettmeria Borgmeier and Moneucoela Kieffer (Díaz and Gallardo 1997, 1998; Gallardo and Díaz 1999). The phylogenies of Fontal-Cazalla et al. (2002) and Buffington et al. (2007) supported the monophyly of the Zaeucoilagroup; as a follow-up, the group was formally recognized as Zaeucoilini by Buffington (2009). The majority of species in this tribe were exclusively Neotropical in distribution, and only Agrostocynips was commonly encountered throughout middle and eastern North America (Forshage et al. 2012) in addition to Central and South America (Buffington and Scheffer 2008).

During the research leading to the publication of Fontal-Cazalla et al. (2002), it was discovered that one taxon included in that work as a Tropideucoila was not actually a member of that genus, and there was no genus to accommodate it; hence, Buffington (2002) erected Aegeseucoela to contain A. flavotincta (Kieffer) and A. grenadensis (Ashmead), two species whose original generic assignment was suspect (Nordlander, pers. comm.). In the years following the publication of Buffington (2009), two issues became clear that would further confuse Zaeucoilini classification if not dealt with: Aegeseucoela flavotincta (Kieffer) warranted its own, new genus, and Aegeseucoela grenadensis (Ashmead) was actually a species of Agrostocynips. As a result, Marthiella Buffington was erected to contain $A$. flavotincta, and the combination $A$. grenadensis was established (and as grenadensis was the type-species of Aegeseucoela, Aegeseucoela became a junior synonym of Agrostocynips) (Buffington 2009). 
In Buffington (2009), it was shown unambiguously that Agrostocynips and Zaeucoila, and the species therein, were difficult to circumscribe, and these taxonomic concepts were more or less unsatisfactory. Agrostocynips was defined by lacking certain characters present in Zaeucoila, a situation that often results in unwieldy keys and frequently indicates paraphyly. The phylogeny presented in Buffington (2009) reflects this, with species of the two genera more or less intertwined in the tree. MLB and FEG, working with newly obtained specimens from throughout the New World, began addressing this problem independently, and have concluded that species of Agrostocynips are Zaeucoila. This revision of the generic boundaries necessitates a redescription of Zaeucoila clarifying the morphological concept. It also provides an opportunity to key and redescribe the included species. The recircumscription also significantly enlarges the distribution area of the genus, which now covers the majority of South and North America, a more extensive distribution than any other Zaeucoiline genus. It is our conviction that the changes made resolves the problems concerning Agrostocynips, and consequently results in clear generic limits in the whole Zaeucoilini. As some species of Zaeucoila have been reared from economically important Agromyzidae (Buffington et al. 2012), we feel this taxonomic clarification, as well as new host and distribution data, might have a positive impact for future research programs in economic entomology and biological control.

The second author noted, based on images supplied by Bob Zuparko (California Academy of Arts and Science, San Francisco), that Rhabdeucoila fulvonotata Kieffer, 1907, belonged in Marthiella, rather than in Tropideucoila where it was placed by Weld (1952) and where it was cited in Buffington (2009); hence we are here making the new combination Marthiella fulvonotata (Kieffer), new combination.

\section{Materials And Methods}

List of depositories.-The specimens used in this study are deposited in the following institutions: AEIC, American Entomological Institute, Gainesville, FL, U.S.A. (D. Wahl); CASC, California Academy of Sciences, San Francisco, CA, U.S.A. (R. Zuparko); CNCI, Canadian National Collection of Insects, Ottawa, Canada (J. Read); MLP, Museo de La Plata, La Plata, Argentina (A. Lanteri); INSUE, Instituto Superior de Entomología "Dr. Abraham Willink", Tucumán, Argentina, (M.V. Colomo); NHMUK, The Natural History Museum, London, U.K. (D. Notton); TAMU, Texas A\&M University Insect Collection, College Station, TX, U.S.A. (E. Riley); UCRC, University of California at Riverside, Riverside, CA, U.S.A. (D. Yanega); USNM, United States National Museum (Smithsonian Institution), Washington, D.C., U.S.A. (M. Buffington); MZSP, Museu de Zoologia da Universidade de São Paulo, SP, Brazil (C.R.F. Brandão); MALUZ, Museo de Artrópodos de la Universidad del Zulia, Maracaibo, Venezuela (J. Camacho).

Specimen illustration and observation.Scanning electron microscope (SEM) images were obtained using a Hitachi TM3000 desktop scanning electron microscope; specimens were shot uncoated at 'analysis' voltage, running in 'compo' mode. Color light microscope images were generated using the EntoVision multiple-focus imaging system. Methods for generating these photographs follow those in Buffington and van Noort (2009). Diffused lighting was achieved using techniques summarized in Buffington et al. (2005), Kerr et al. (2009) and Buffington and Gates (2009). Resulting images were 
edited in Adobe CS4. All images are available from Morphbank.

Descriptive format.-Specimen-level data for all specimens of each species were uploaded to vSyslab (Johnson 2008), and these data were linked to species concepts in the database. For each species concept, a matrix of characters was coded, and this matrix of characters were used to automatically generate the descriptions in natural language. Only species-level characters are focused on in the species descriptions; generic-level characters are covered in the redescription of the genus (which largely follows that of Buffington (2009)), and are not repeated for each species. Morphological terminology follows Buffington (2009); surface sculpture terminology follows that of Harris (1979).

\section{Systematic Treatment}

Zaeucoila Ashmead, 1903

Zaeucoila Ashmead, 1903a: 222; Dalla Torre and Kieffer, 1910: 102, 891; Rohwer and Fagan, 1917: 378; Weld, 1921: 444, 1952: 105, 193 (redescription); Borgmeier, 1935: 116; Nordlander 1982: 16, 21-22; Díaz \& Gallardo, 1997: 34 (revision), 1998: 111; Gallardo \& Díaz, 1999: 20; Marchiori \& Oliveira, 2001: 337; Fontal-Cazalla et al., 2002: 179; Selegatto et. al., 2004: 624; Fontal and Nieves-Aldrey, 2004: 64, 77; Buffington, 2009: 184-185 (redescription).

Zaeucoela Ashmead, 1903b: 66, 71. Misspelling.

Agrostocynips Díaz, 1976: 33 (in De Santis et al.); new synonymy.

Aegeseucoela Buffington, 2002: 593. Synonymy by Buffington (2009).

Type species._Zaeucoila unicarinata Ashmead, 1903a: 222, by original designation.

Redescription.-Head: Nearly glabrous with scattered setae along lower face, clypeus and gena; ocelar hair patch absent (only present in $Z$. grenadensis). Orbital furrows originating at torulus, terminating at malar sulcus. Malar sulcus simple. Malar space smooth; ventral margin with a single prominent conical protuberance. Genal carina running from malar space to dorsal margin of compound eyes, or only along ventral margin of malar space.

Antenna: Female: 13 segments, semiclavate; segments 3-13 of subequal size; placoidal sensillae present on segments 2-13, 3-13, 4-13 or 5-13. Male: 15 segments, 4-15 of subequal size; placoidal sensillae present on segments 3-15, segment 3 modified, slightly longer than 4 , curved, excavated laterally. In both sexes, segments pubescent, subcircular in crosssection.

Pronotum: Pronotal plate wide, with setae along the dorsal margin, dorsal margin marginate. Pronotal fovea open. Pronotal triangle absent or present. Pronotal impression absent. Lateral pronotal carina absent. Lateral aspect of pronotum glabrous, smooth.

Mesoscutum: Mesoscutal carina absent or present, at least anteriorly, tapering towards posterior margin of mesoscutum. Parascutal impression incomplete, narrow. Notauli, parapsidal ridges and parapsidal hair lines usually absent.

Mesopectus: Upper part and lower part of mesopleuron smooth and glabrous. Dorsal margin of mesopleural triangle well defined, rounded ventrally. Mesopleural carina simple. Lower part of mesopleuron bounded by distinct precoxal carina.

Scutellum: Scutellar plate ranging from smaller to large; posterior margin rounded or truncate. Glandular release pit placed in the center or between center point of plate and posterior margin of plate; rim of plate translucent; tubercles commonly found along the entire rim. Dorsal surface of scutellum either areolate/foveate or having large, radiating ridges, rounded laterally and posteriorly, laterodorsal and 
posterior projections of scutellum absent. Lateral bars as long as wide; ventral lobe present, scutellar fovea oval, smooth and deep.

Metapectal-propodeal complex: Anterior part of complex glabrous, posterior part setose. Spiracular groove with a well defined dorsal margin, ventral margin sometimes reduced. Metapleural ridge reduced to absent, submetapleural ridge absent. Anterior impressions of metepimeron and metepisternum present. Anteroventral cavity semioval, setose. Propodeum covered in both long and short setae. Lateral propodeal carinae semiparalell, bowed at junction with the auxiliary propodeal carinae; propodeal carinae reduced. Nucha glabrous, crenulate or reticulate.

Wings: Base of wing hyaline or infuscate, setose or glabrous. R1 complete. Marginal cell typically as long as deep, open or closed. Apical fringe present, medium in length.

Legs: Fore and mid coxa subequal in size, hind coxa twice the size of either fore or mid coxa. Fore coxa variably setose; mid and hind coxa with distinct lateral and posterior dorsoventral lateral setals bands. Femora and tibiae sparsely setose; tarsomeres with dense setae.

Metasoma: Female: subequal in size to mesosoma. Base of syntergum with hairy ring present, remainder of metasoma glabrous. Terga posterior to syntergum directed posteroventrally, resulting in a $70^{\circ}$ or in a near $90^{\circ}$ degree angle between syntergum and terga. Male: as in female with terga posterior to syntergum abruptly angled ventrally, resulting in a $90^{\circ}$ angle between syntergum and remaining terga. Micropunctures present on posterior quarter of syntergum, and on remaining terga.

Biology.-Koinobiont endoparasitoids of Agromyzidae (Diptera). The host is parasitized while still in the leaf, typically in the first or second instar. The parasitoid consumes the host after the host has dropped from the host plant and created the puparium in the soil below. The parasitoid later emerges from the puparium, in the soil, below the host plant.

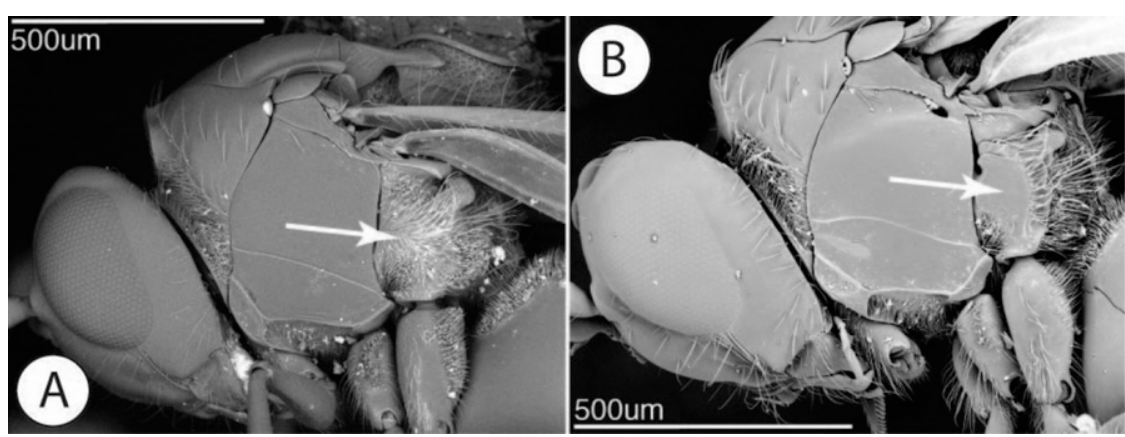

1. Metapleuron entirely setose (arrow, A) Zaeeucoila normae n. sp.

- Metapleuron, at most, with posterior half of metapleuron setose; -remainder of metapleuron glabrous (arrow, B). 


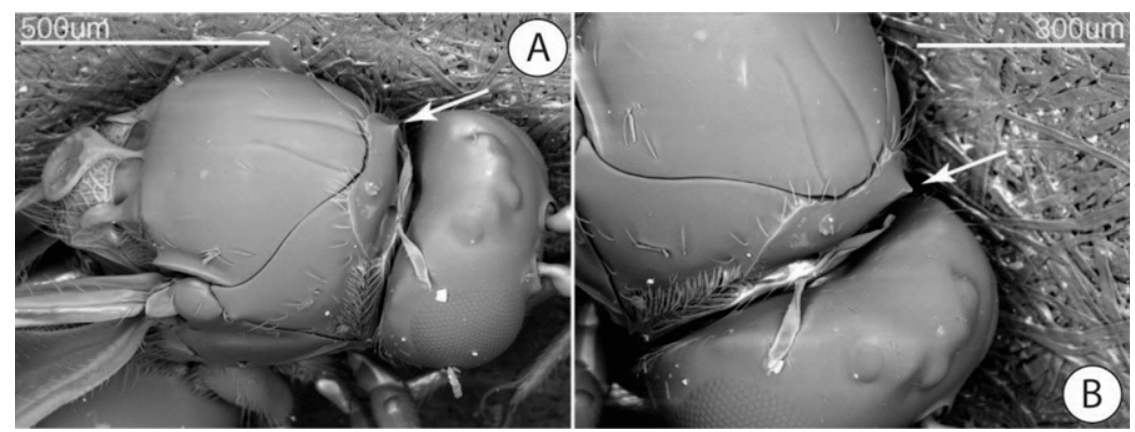

2. Anterior face of pronotal plate with a pair of forward-projecting conical protuberances (arrows, A and B) Zaeucoila bituberculata n. sp.

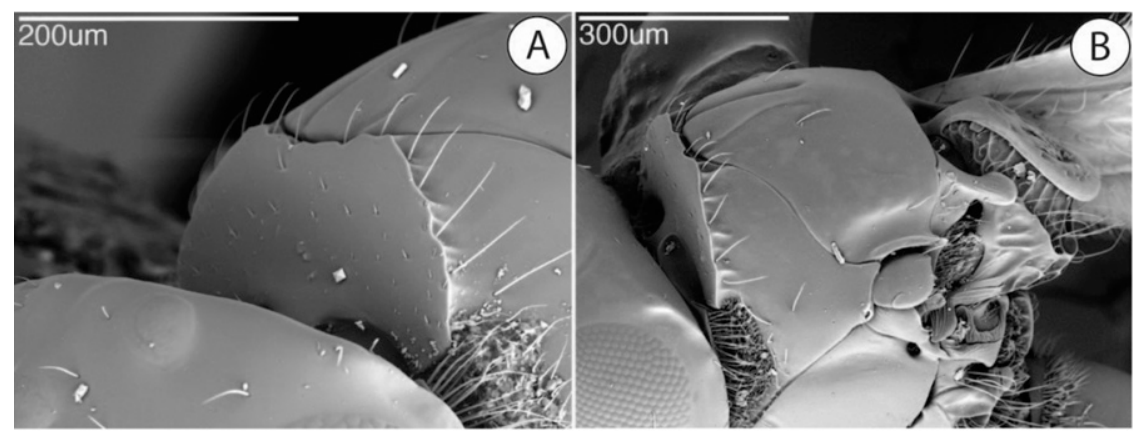

- Anterior face of pronotal plate entirely flat, no conical protuberances present (A and $\mathrm{B}$ ) ..... 3

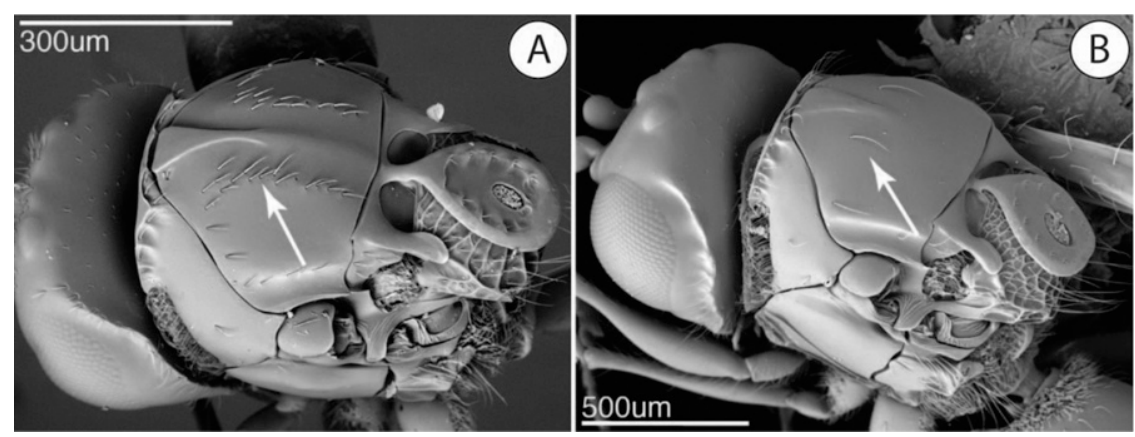

3. Mesoscutum with a well developed mesocutal keel and dense setal tracks parallel to the keel (A) Zaeucoila grenadensis

- Mesoscutum without dense setal tracks, with at most, thin setal tracks; mesoscutal keel present to absent (B).. 


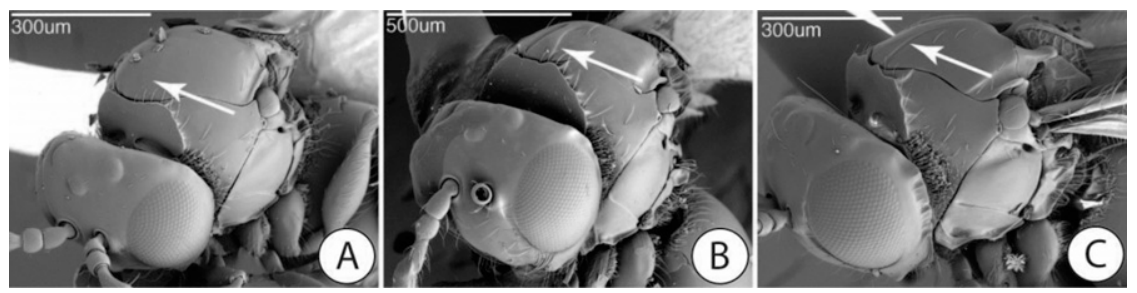

4. Mesoscutum with no trace of admedial lines or mesocutal keel (arrow, A) .5

- Mesoscutum ranging from distinct admedial lines (arrow, B) to a well-developed mesoscutal keel (arrow, C). .8

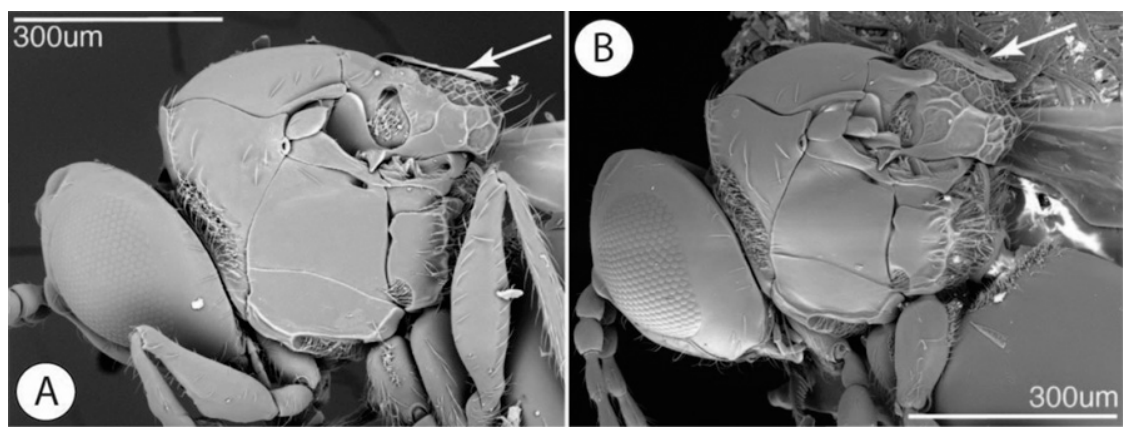

5. Scutellum, when viewed laterally, at the same level as mesoscutum (scutellum flattened) (arrow, A) Zaeucoila patera n. sp.

- Scutellum, when viewed laterally, distinctly rising above the level of the mesoscutum (arrow, B)
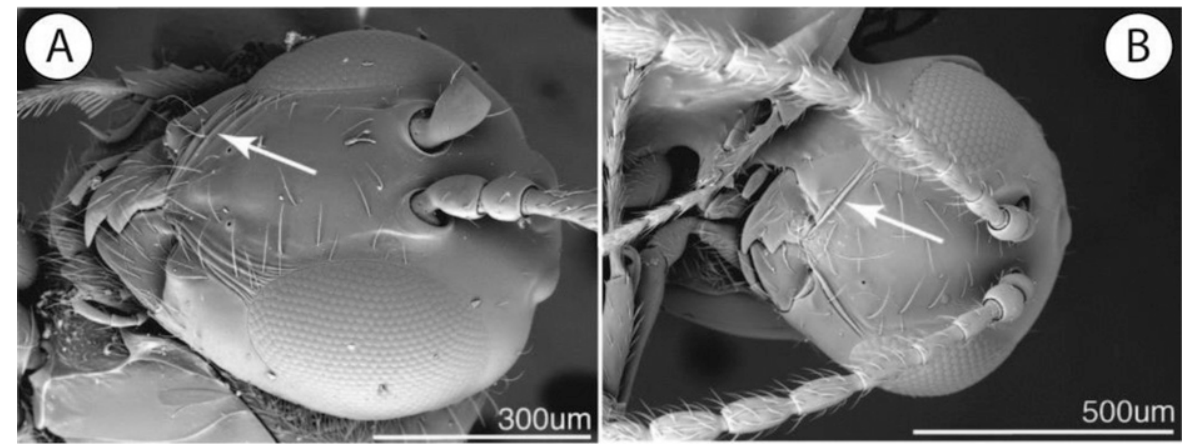

6. Malar sulcus composed of a series of striae (arrow, A) Zaeucoila fidalgoii n. sp.

- Malar sulcus simple, composed of a single or two striae (arrow, B).... 7. 

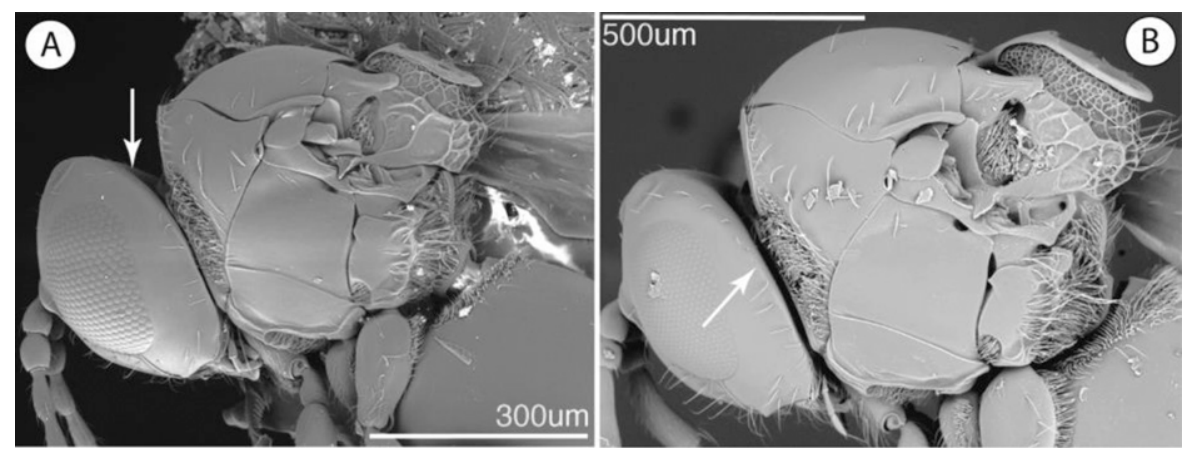

7. Genal carina clearly present, extending beyond dorsal margin of eye (seen in lateral view) (arrow, A). Zaeucoila triangulifera

- Genal carina apparently absent; when visible, the genal carina can only be seen extending to, at most, halfway up the length of the eye (seen in lateral view) (arrow, B)...... Zaeucoila robusta

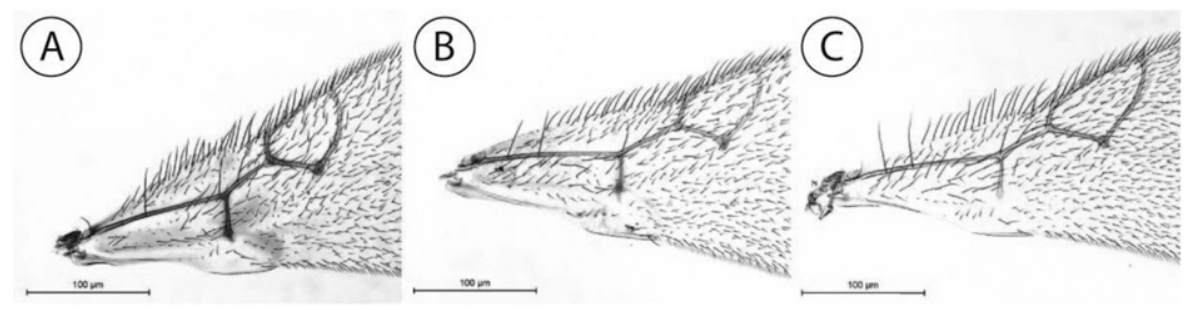

8. Base of forewing with at least some indication of infuscation (A and B)............................

- Base of forewing entirely clear, no indication of any infuscation $(\mathrm{C})$................................... 11 


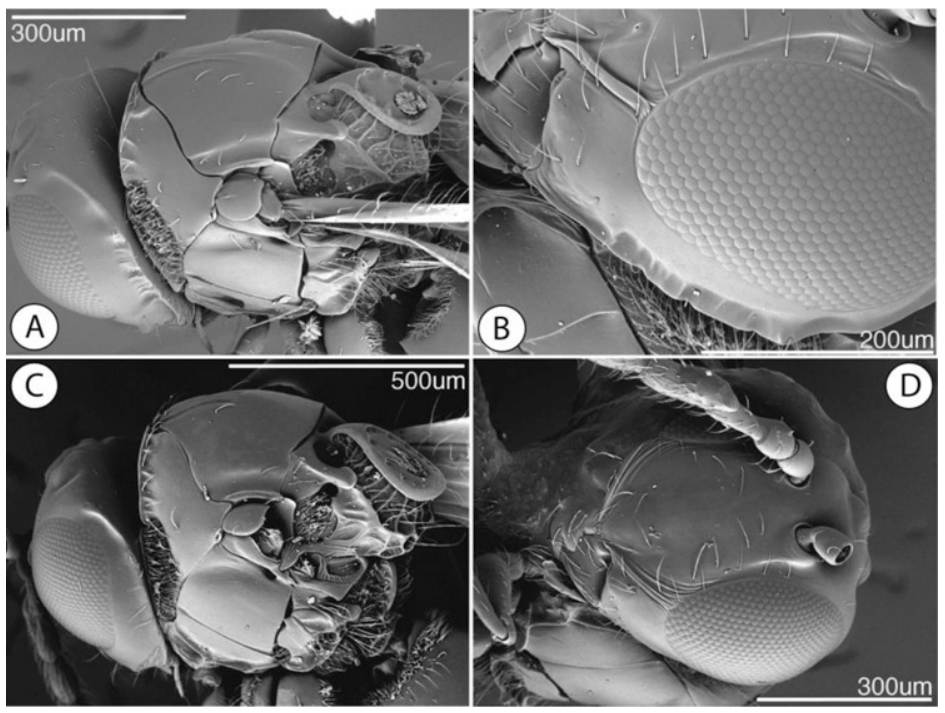

9. Inner orbital furrow sharply defined along its entire length (B); malar sulcus simple, composed of single ridge (B); sparse setal tracks usually present on mesoscutum (A); genal carina always heavily scalloped (A and B); mesoscutal keel frequently reaching past the midline of the mesoscutum (A)......

Z. johnsonii n. sp.

- Inner orbital furrow defined dorsally near toruli, less so ventrally (D); malar sulcus typically composed of a series of striae, especially at the end meeting the eye (D); no setal tracks present on mesoscutum (C); genal carina typically smooth, sometimes scalloped (C); mesoscutal carina extending, at most, to midline of mesoscutum (C)

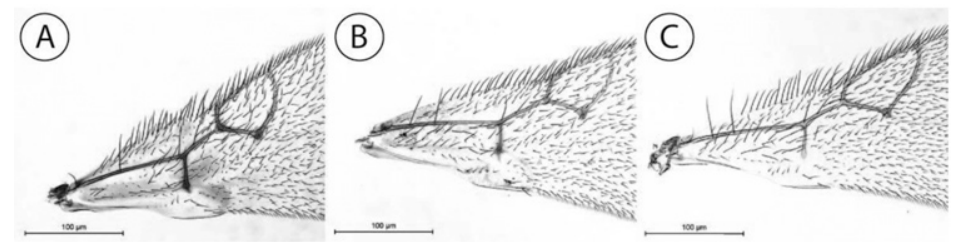

10. Area at base of forewing with broad areas of distinct infuscation, sometimes quite dark (A and B).

- Area at base of forewing hyaline, with no visible infuscation 13

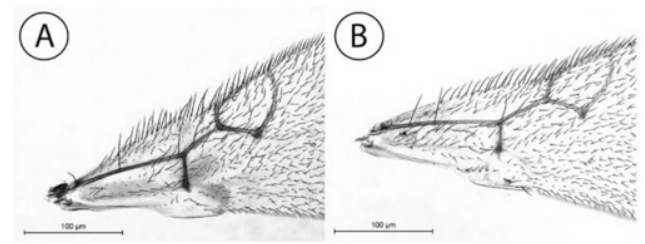

11. Area at base of forewing with broad areas of distinct infuscation, sometimes quite dark (A) Zaeucoila infuscata n. sp.

- Area at base of forewing with limited infuscation, specifically restricted to space surrounding the wing veins $(\mathrm{B})$. 

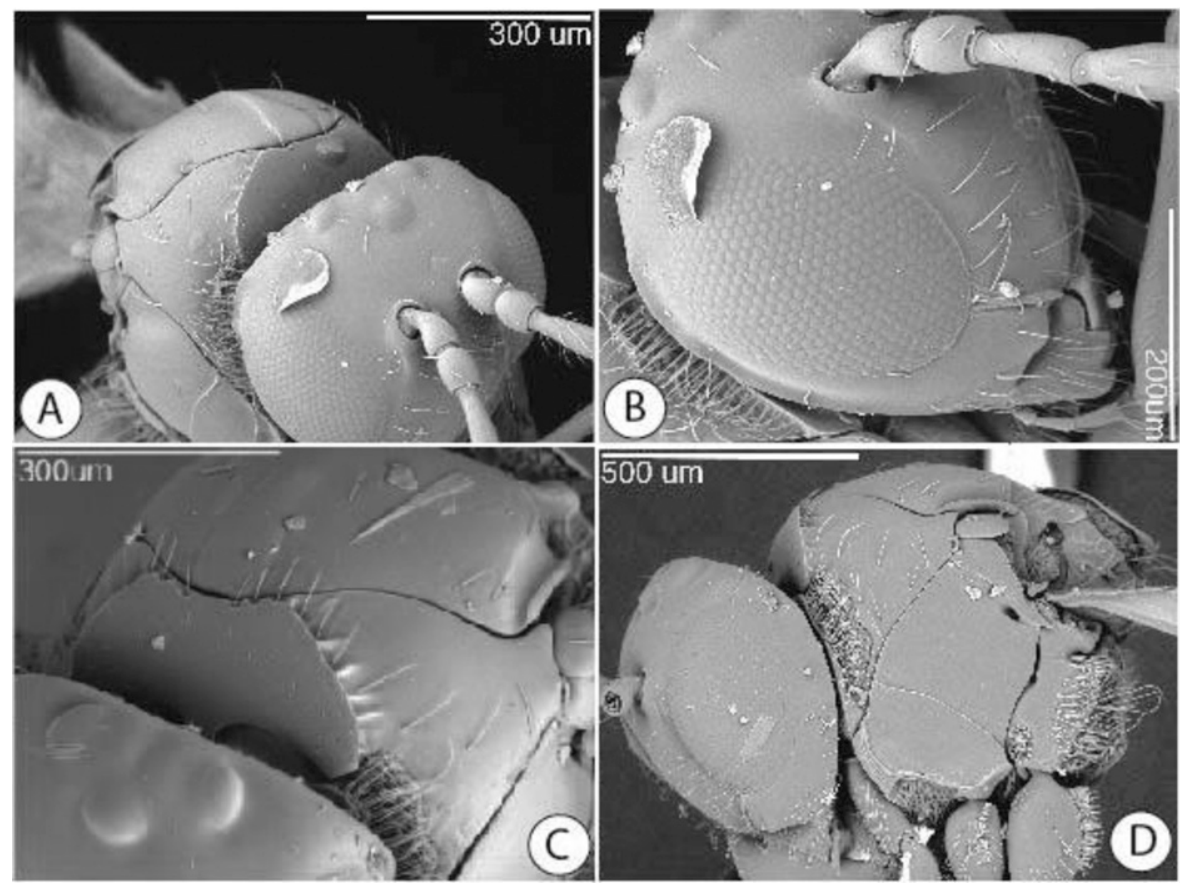

12. Mesoscutal carina extending from the pronotal plate to at least one-half the length of the mesoscutum (A); orbital grooves distinctly present along the medial edge of the compound eye (B); malar sulcus simple, composed of a few striae merging into a single ridge (B); genal carina most often distinctly scalloped (A and B).

Zaeucoila unicarinata

- Mesoscutal carina typically absent; if present, only indicated anteriorly behind the pronotal plate $(\mathrm{C})$, never reaching half-way across the mesoscutum; orbital grooves present but weakly indicated along medial edge of compound eye (D); malar sulcus composed of a series of striae (D)......................................... Zaeucoila lignys n. sp.
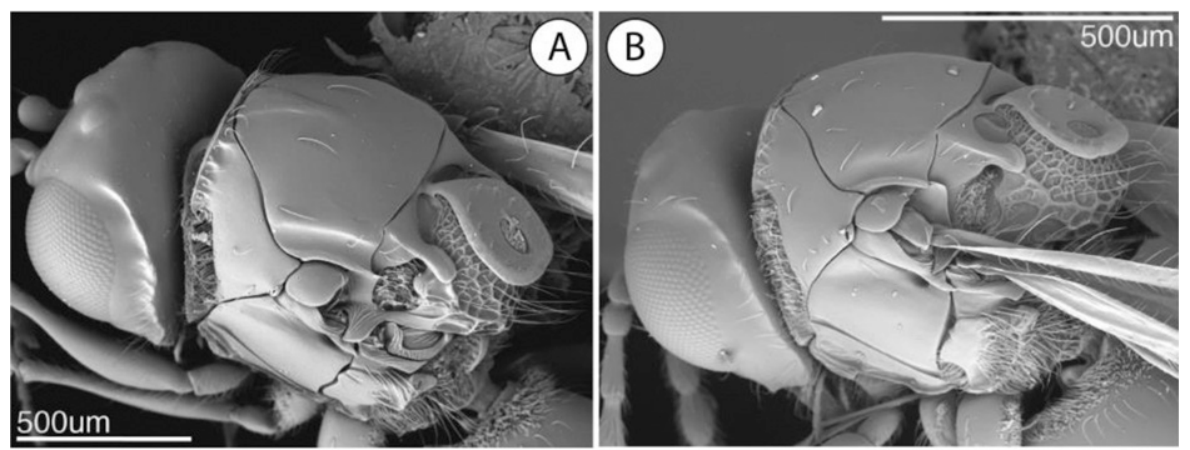

13. Legs entirely honey yellow; two long seta present on either side of the mesoscutal keel about halfway along its length $(\mathrm{A})$; genal carina distinctly scalloped. Zaeucoila flavipes.

- Base of legs dark brown to mahogony in color, remaining segments orange-yellow; one, sometimes two short seta present on either side of the mesoscutal keel, along with other short setae (B); genal carina most often smooth, occasionally gently scalloped......

Zaeucoila incompleta 
Distribution.--Southern Canada, USA, Central America, South America, Carribbean (Buffington 2004, 2009; De Santis et al. 1975, 1976; Díaz and Gallardo 1997; Fontal and Nieves-Aldrey 2004). A summary map of all species of Zaeucoila can be found here: http://hol.osu.edu/map-full. html?id=244108.

\section{DESCRIPTIONS AND REDESCRIPTIONS}

\section{Zaeucoila bituberculata Buffington and Gallardo, new species}

urn:1sid:zoobank.org:act:576ACC14-BEE54CD0-84F9-9E8CF399D7B8

http://bioguid.osu.edu/xbiod_concepts/407938

(Figs. 2-7)

Description.-Coloration with head, mesosoma, metasoma black to dark brown; legs with coxae and femora dark brown, rest yellow. Lateral margin of occiput defined by evenly rounded, raised, sharp carina. Vertical carina adjacent to ventral margin of antennal socket present, leading to shallow inner orbital groove. Malar space adjacent to anterior articulation of mandible with distinct conical protuberance, smooth. Malar sulcus present, composed of single line. Orbital furrows rounded, ill defined, running from lateral edge of torulus to ventral margin of compound eye. Dorsal aspect of vertex smooth with a few short, scattered setae. Posterior aspect of vertex smooth with fine setae. Hair punctures on lateral aspect of vertex absent. Placoidal sensilla present on F3-11. Pubescence on lateral surface of pronotum present, sparse, consisting of few short hairs. Ridges extending posteriorly from lateral margin of pronotal plate absent. Sculpture on mesoscutum absent, entire surface smooth, shiny. Median mesoscutal carina present, composed of anteriorly broad elevation, narrowing posteriorly, ending at midline of mesoscutum. Anterior admedial lines present, in conjunction with mesoscutal carina. Lateroventral mesopleural carina present, marking abrupt change of slope of mesopectus, terminating anteriorly at anterior flange of mesosubpleuron. Mesopleural triangle present, gently impressed lacking dorsal and ventral border; smooth. Dorsal surface of scutellum foveateareolet. Latero-ventral margin of scutellum posterior to auricula smooth, with weak longitudinal striae. Subalar area abruptly broadened anteriorly, with an indicated longitudinal division. Pubescence present along posterior and ventral margins of metapleuron, long, dense; long and thin on propodeum. Pubescence of fore wing absent on mesal half of wing, sparse distally. Coloration of wing absent, entire wing hyaline. Hair fringe along apical margin of fore wing present, very short.

Inter propodeal carinae space smooth with confined setal patch.

Diagnosis.-This species is unique with the possession of twin conical protuberances on the anterior face of the pronotal plate. This character state has not been observed in any other eucoiline species.

Etymology.-Name refers to the twin corniculae on the pronotal plate.

Link to Distribution Map.-[http:// hol.osu.edu/map-full.html?id=407938]

Material Examined.-Holotype, female: MARTINIQUE: Trinité Arrond., MP 49, L'Ajoupa-Bouillon, 26.IV.1994, reared, J. Etienne, USNMENT00977920 (deposited in USNM).

Distribution.-Neotropical Region: Martinique.

Biology.-Host not observed.

\section{Zaeucoila fidalgoi Buffington and Gallardo, new species}

urn:1sid:zoobank.org:act:A48570CB-

C568-4C1E-96AA-A7D8FA188516

http://bioguid.osu.edu/xbiod_concepts/410040

(Figs. 8-13) 


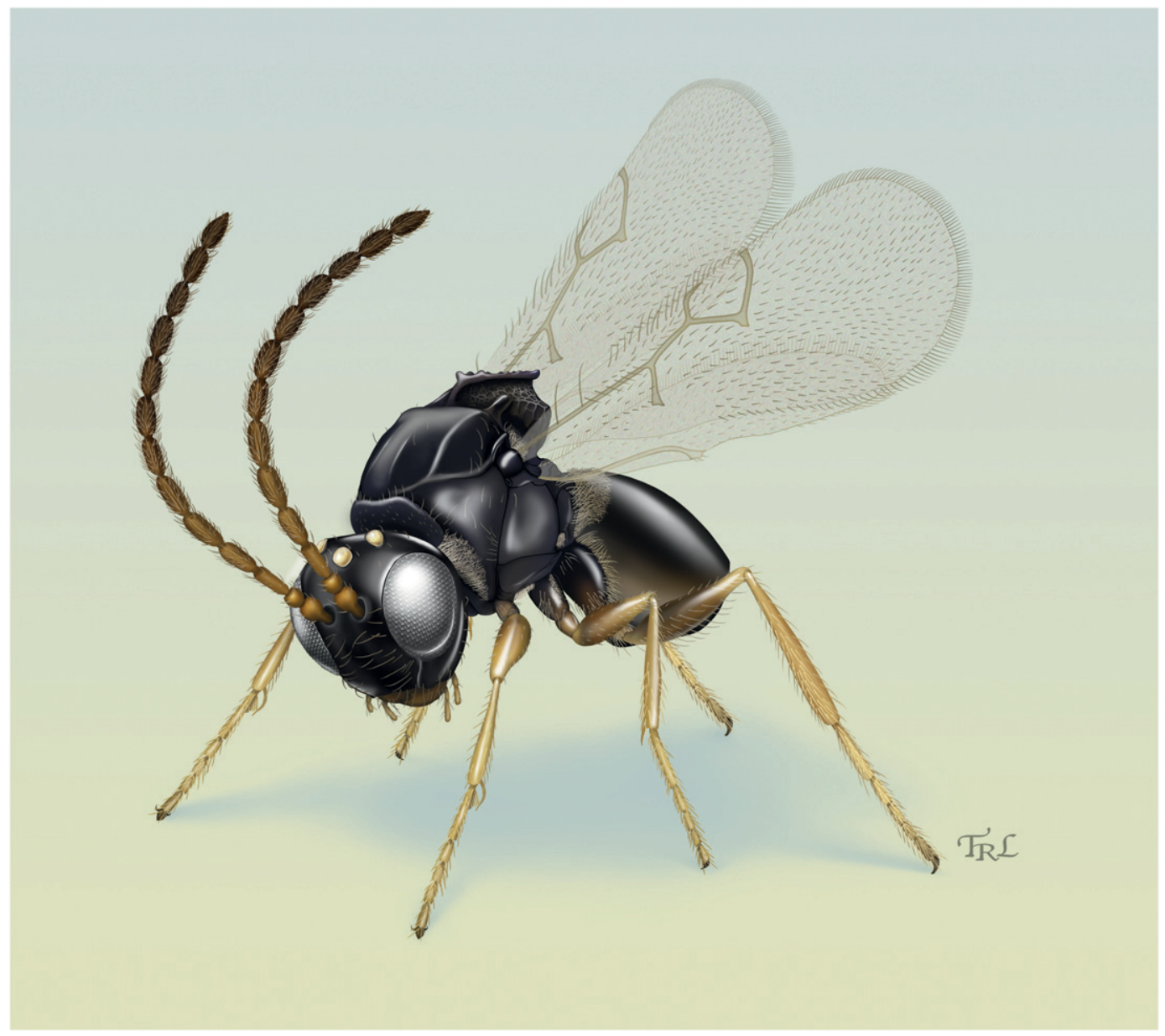

Plate 1, Fig. 1. Digital painting of a female Zaeucoila robusta.

Description.-Coloration with head, mesosoma, metasoma black to dark brown; legs with coxae and femora dark brown, rest yellow. Lateral margin of occiput defined by distinctly angled, raised, sharp carina. Vertical carina adjacent to ventral margin of antennal socket present, leading to shallow inner orbital groove. Malar space adjacent to anterior articulation of mandible with distinct conical protuberance, smooth. Malar sulcus present, composed of series of small striae. Orbital furrows rounded, divergent ledges running from antennal sockets to dorsal end of malar sulcus. Dorsal aspect of vertex smooth with a few short, scattered setae. Posterior aspect of vertex smooth with fine setae. Hair punctures on lateral aspect of vertex absent. Placoidal sensilla present on F2-11. Pubescence on lateral surface of pronotum present, sparse, consisting of few short hairs. Ridges extending posteriorly from lateral margin of pronotal plate distinct but short, not extending to the dorsal margin of pronotum. Sculpture on mesoscutum absent, entire surface smooth, shiny. Median mesoscutal carina absent. Anterior admedial lines absent. 

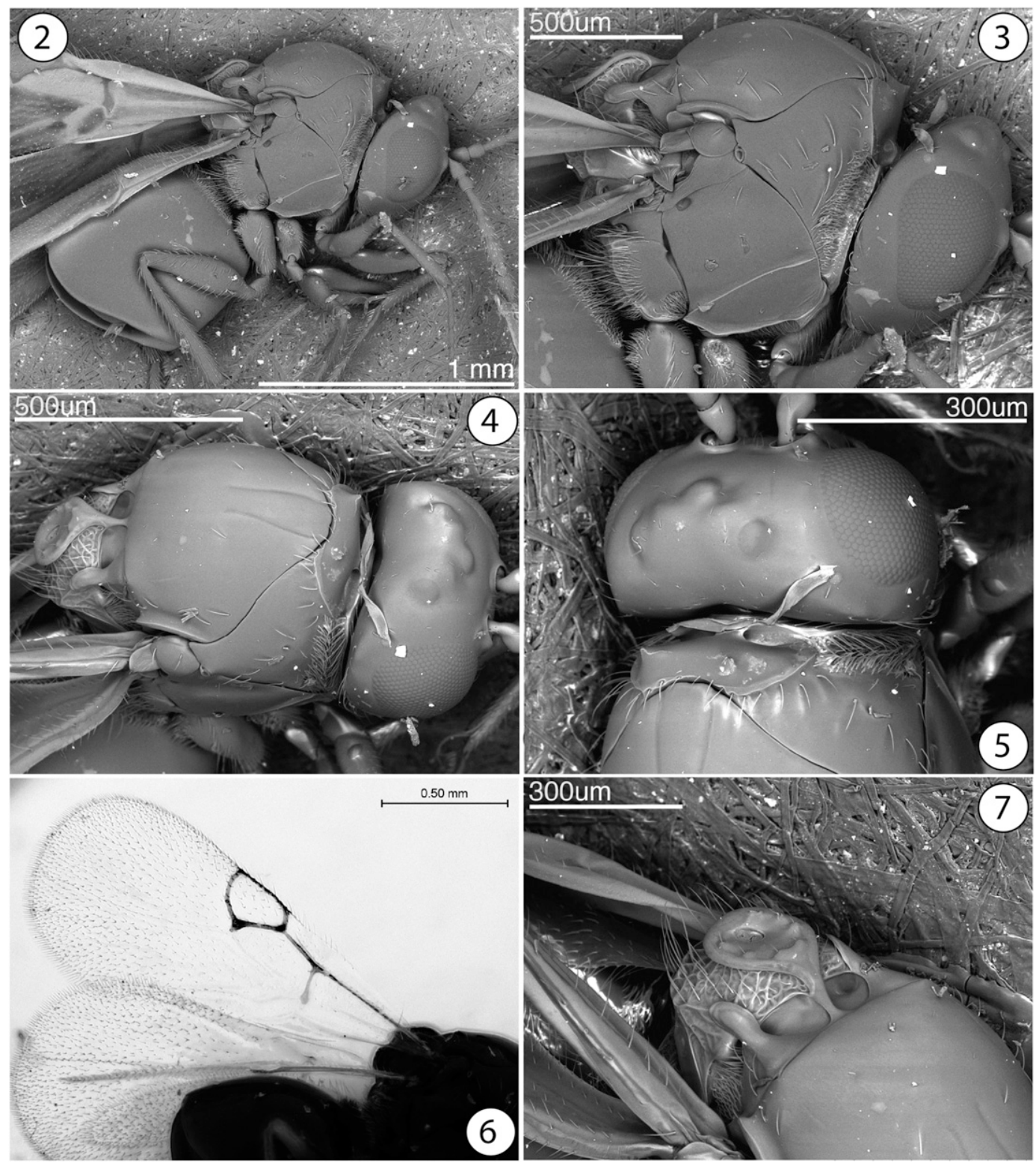

Plate 2, Figs. 2-7. Zaeucoila bituberculata, new species. 10, habitus, lateral; 11, head and mesosoma, lateral; 12, head and mesosoma, dorsal; 13, head and pronotum, dorsal; 14, forewings; 15, scutellum, dorsal view.

Lateroventral mesopleural carina present, marking abrupt change of slope of mesopectus, terminating anteriorly at large, oblique notch. Mesopleural triangle present, gently impressed with distinct dorsal border, ventral border lacking; smooth. Dorsal surface of scutellum foveate-areolet. Latero-ventral margin of scutellum posterior to auricula smooth ventrally, longitudinally striate dorsally. Subalar area slightly broadened anteriorly, with distinct laterally protruding lobe 

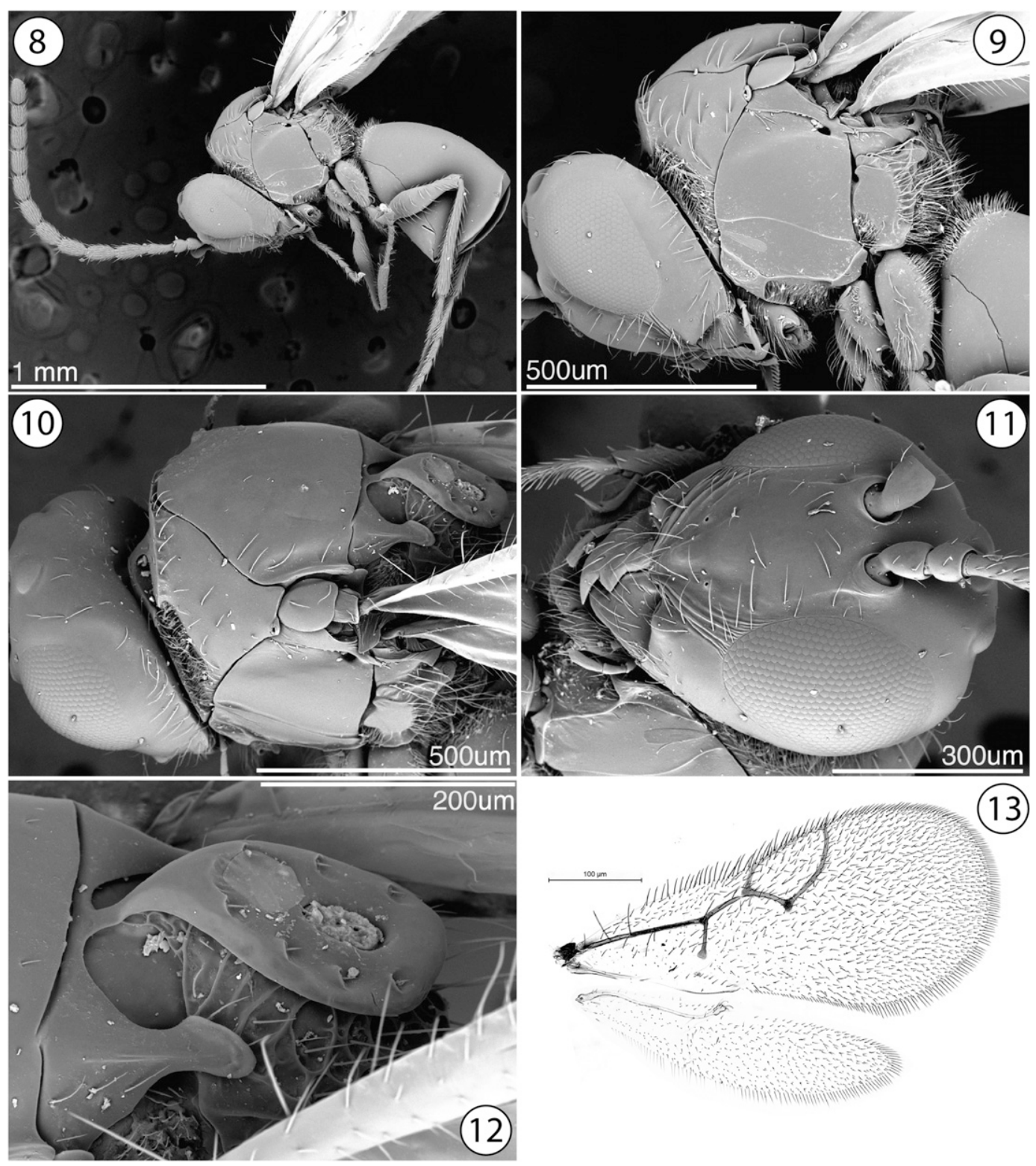

(13)

Plate 3, Figs. 8-13. Zaeucoila fidalgoii, new species. 16, habitus, lateral view; 17, head and mesosoma, lateral view; 18, head and mesosoma, dorsal view; 19, head, anterior view; 20, scutellum, dorsolateral view; 21 , fore and hind wings.

ventrally. Pubescence consisting of few hairs on posterior part of metaplueron, dense hair on propodeum. Pubescence of fore wing mesally sparse, gradually becoming denser distally. Coloration of wing absent, entire wing hyaline. Hair fringe along apical margin of fore wing present, with particularly longer setae along the mesal anterior margin. Inter propodeal carinae space moderately 


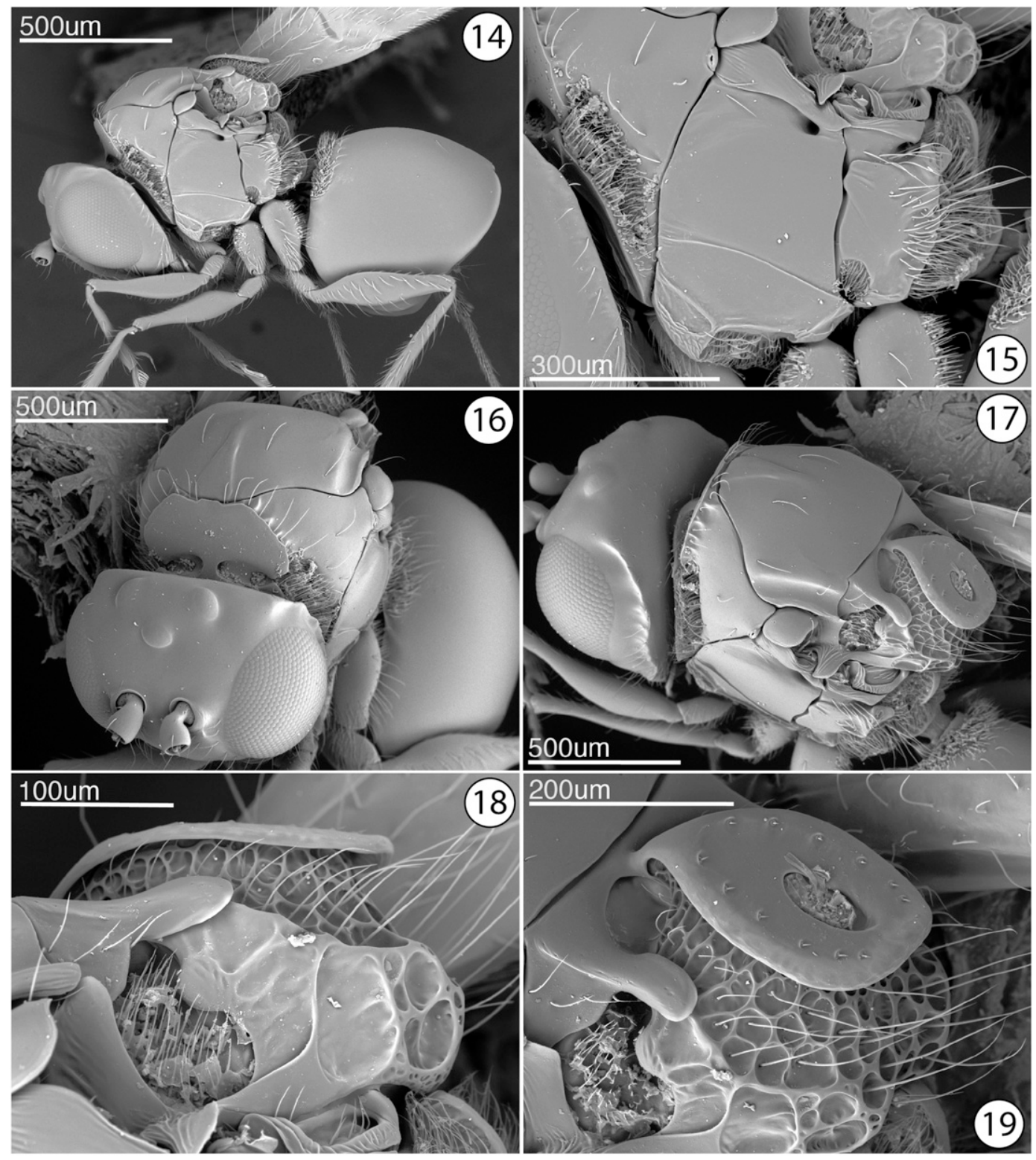

Plate 4, Figs. 14-19. Zaeucoila flavipes, new combination. 2, habitus; 3, mesosoma, lateral view; 4, head and mesosoma, anterodorsal view; 5, head and mesosoma, laterodorsal view; 6, scutellum, lateral view; 7, scutellum, posterolateral view.

setose, with long, thin setae evenly filling the space; surface underneath smooth.

Diagnosis.-Many Zaeucoila have a series of striae that compose the malar sulcus at the end connecting to the compound eye, but these striae merge to a single, or at most, double sulcus. In the case of $Z$. fildalgoii, the malar sulcus is entirely composed of a series of striae, with no single dominant ridge. The marginal cell of the forewing is 

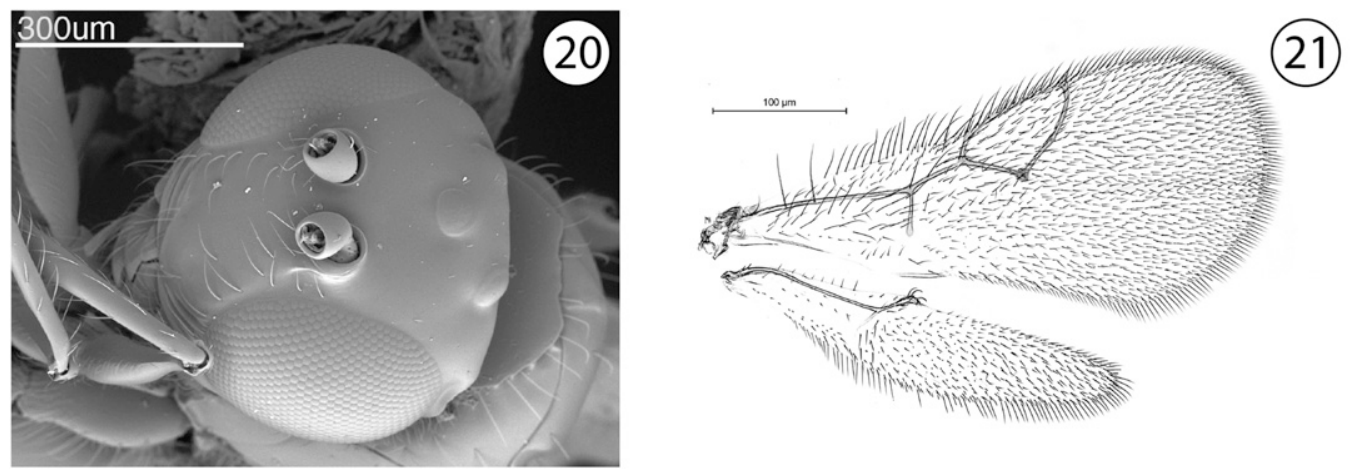

Plate 5, Figs. 20-21. Zaeucoila flavipes, new combination. 8, head, anterior view; 9, fore and hind wings.

somewhat unique, too, in that its length is much greater than its depth, resulting in what appears to be a very large marginal cell for the size of the wing.

Etymology.-Named in honor of the eminent hymenopterist and collector of this species, Dr. Patricio Fildalgo (CONICET, Anillaco, La Rioja, Argentina).

Link to Distribution Map.-[http:// hol.osu.edu/map-full.html?id=410040]

Material Examined.-Holotype, male: ARGENTINA: La Rioja Prov., Santa Cruz, 15.IX-14.X.2002, Fidalgo, USNMENT00993727 (deposited in USNM). Paratypes: (1 female, 5 males) ARGENTINA: La Rioja Prov., Santa Cruz, 15.IX-14.X.2002, Fidalgo (5 males, USNMENT00993716, 00993725, 00993729, 00993733, 00993740 (USNM)). COSTA RICA: Guanacaste Prov., H.3.O, Santa Rosa National Park, 300m, 11.V-1.VI.1985, Malaise trap, D. Janzen \& I. Gauld (1 female, USNMENT01223590 (USNM)).

Distribution.-Neotropical Region: Argentina and Costa Rica.

Biology.-Unknown.

\section{Zaeucoila flavipes (Ashmead), new combination}

http://bioguid.osu.edu/xbiod_concepts/ 386611

(Figs. 14-21)
Chrestosema flavipes Ashmead, 1896: 753 (original description).

Description.-Coloration. Lateral margin of occiput defined by scalloped, sharp carina. Vertical carina adjacent to ventral margin of antennal socket present, leading to shallow inner orbital groove. Malar space adjacent to anterior articulation of mandible with distinct pyramidal protuberance, smooth. Malar sulcus present, composed of series of small striae. Orbital furrows rounded, ill defined, running from lateral edge of torulus to ventral margin of compound eye. Dorsal aspect of vertex smooth with a few short, scattered setae. Posterior aspect of vertex smooth with fine setae. Hair punctures on lateral aspect of vertex absent. Placoidal sensilla present on F2-11. Pubescence on lateral surface of pronotum present, sparse, with distinct row behind pronotal plate. Ridges extending posteriorly from lateral margin of pronotal plate present, typically short, with single prominent ridge extending to anterior margin of mesoscutum. Median mesoscutal carina present, composed of anteriorly broad elevation, narrowing posteriorly, ending at midline of mesoscutum, with four long, slender setae, one pair on each side of mesoscutal carina. Anterior admedial lines present, in conjunction 
with mesoscutal carina. Lateroventral mesopleural carina present, marking abrupt change of slope of mesopectus, terminating anteriorly at large, oblique notch. Mesopleural triangle present, distinctly impressed with distinct dorsal and ventral border, with single, long seta. Dorsal surface of scutellum foveate-areolate. Latero-ventral margin of scutellum posterior to auricula smooth ventrally, longitudinally striate dorsally. Subalar area slightly broadened anteriorly, with an indicated longitudinal division. Pubescence consisting of few hairs on posterior part of metapleuron, dense hair on propodeum. Pubescence of fore wing mesally sparse, gradually becoming denser distally. Coloration of wing absent, entire wing hyaline. Hair fringe along apical margin of fore wing present, with particularly longer setae along the mesal anterior margin. Inter propodeal carinae space moderately setose, with short, thin setae evenly filling the space; surface underneath smooth.

Diagnosis._Zaeucoila flavipes has a distinctly scalloped genal carina, shared only with Z. unicarinata and Z. johnsonii. Zaeucoila flavipes differs from these two species by the possession of two distinct, long, pairs of setae, one pair on each side of the mesoscutal keel. This species may also be confused with Z. incompleta, as some specimens of this latter species have been observed with a partially scalloped genal carina. However, in these specimens, the paired setae are not present, but instead, there are more mesoscutal setae scattered across the mesoscutum.

Link to Distribution Map.-[http:// hol.osu.edu/map-full.html?id=409614]

Material Examined.-Holotype, female: Grenada, West Indies, Mount Gay Estate (Leeward side), H.H. Smith '29' [first label]; W. Indies 99-331 [second label] Chrestosema flavipes Ashm female type (in Ashmead's hand, third label)]; Holotype, B.M. TYPE HYM 7.92 (fourth label)]; NHMUK 010815900 (fifth label). Other material: (72 females, 20 males) ARGENTINA: Salta Prov., $781 \mathrm{~m}, 24^{\circ} 47^{\prime} 38^{\prime \prime} \mathrm{S} 6^{\circ} 01^{\prime} 56^{\prime \prime} \mathrm{W}$, Cabeza de Buey, 21.III.2003, sweeping, J. Munro (1 female, USNMENT00977609 (USNM)). Salta Prov., Aguas Blancas, along Bolivian border, rainforest, $447 \mathrm{~m}, 22.72^{\circ} \mathrm{S} 64.40^{\circ} \mathrm{W}$, Provincial Route 19, 23.III.2003, sweeping, J. Munro (1 female, USNMENT00977607 (USNM)). BOLIVIA: Santa Cruz Dept., $18^{\circ} 06^{\prime} \mathrm{S} 63^{\circ} 36^{\prime} \mathrm{W}$, El Refugio Los Volcanes, 25.X-30.X.2007, Malaise trap, J. Romero \& F. Romero (1 male, USNMENT00977967 (USNM)). COLOMBIA: Magdalena Dept., Borde camino Pueblito-Cañaveral, M.3268, 30m, $11^{\circ} 20^{\prime} \mathrm{N}$ $74^{\circ} 02^{\prime} \mathrm{W}$, Tayrona Natural National Park, 18.VII-25.VII.2002, sweeping, M. Sharkey, D. Arias \& E. Torres (1 male, USNMENT00977885 (USNM)). Magdalena Dept., Pueblito, M.944, 225m, $11^{\circ} 20^{\prime} \mathrm{N}$ $74^{\circ} 02^{\prime} \mathrm{W}$, Tayrona Natural National Park, 26.X-22.XI.2000, Malaise trap, R. Henriquez (1 female, USNMENT00977866 (USNM)). Magdalena Dept., Tayrona Natural N.P., M.131, 30m, $11^{\circ} 20^{\prime} \mathrm{N}$ $74^{\circ} 02^{\prime} \mathrm{W}$, Cañaveral, 26.IV-12.V.2000, Malaise trap, R. Henriquez (1 male, USNMENT00977442 (UCRC)). Magdalena Dept., Tayrona Natural N.P., M.627, $30 \mathrm{~m}, 11^{\circ} 20^{\prime} \mathrm{N} 74^{\circ} 02^{\prime} \mathrm{W}$, Cañaveral, 30. VIII-19.IX.2000, Malaise trap, R. Henriquez (5 females, 2 males, USNMENT0097789200977898 (USNM)). Magdalena Dept., Zaino, M.136, 50m, $11^{\circ} 20^{\prime} \mathrm{N} 74^{\circ} 02^{\prime} \mathrm{W}$, Tayrona Natural National Park, 3.V-30.V.2000, Malaise trap, R. Henriquez (1 male, USNMENT00977443 (UCRC)). Magdalena Dept., Zaino, M.789, 50m, $11^{\circ} 20^{\prime} \mathrm{N}$ $74^{\circ} 02^{\prime} \mathrm{W}$, Tayrona Natural National Park, 10.X-3.XI.2000, Malaise trap, R. Henriquez (3 males, USNMENT0097790000977902 (USNM)). Magdalena Dept., Zaino, M.793, 50m, $11^{\circ} 20^{\prime} \mathrm{N} 74^{\circ} 02^{\prime} \mathrm{W}$, Tayrona Natural National Park, 29.IX-17. 
X.2000, Malaise trap, R. Henriquez (1 male, USNMENT00977813 (USNM)). Magdalena Dept., Zaino, M.967, 50m, $11^{\circ} 20^{\prime} \mathrm{N} 74^{\circ} 02^{\prime} \mathrm{W}$, Tayrona Natural National Park, 15.XII-2.I.2001, Malaise trap, R. Henriquez (3 females, 1 male, USNMENT00993838, 00993840-00993842 (USNM)). Magdalena Dept., road between Cañaveral and Arrecifes, M.4172, $50 \mathrm{~m}, 11^{\circ} 18^{\prime} \mathrm{N} 73^{\circ} 56^{\prime} \mathrm{W}$, Tayrona Natural National Park, 21.VII-23.VII.2002, sweeping, M. Sharkey, D. Arias \& E. Torres (1 male, USNMENT00977886 (USNM)). Meta Dept., Cabaña Las Mirlas, M.4349, 710m, $03^{\circ} 48^{\prime} \mathrm{N} 73^{\circ} 52^{\prime} \mathrm{W}$, Sumapaz Natural National Park, 19.X-5. XI.2003, Malaise trap, A. Torrijos (1 female, USNMENT00977884 (USNM)). Valle del Cauca Dept., Anchicaya, M.1110, 730m, $03^{\circ} 26^{\prime} \mathrm{N} 76^{\circ} 48^{\prime} \mathrm{W}$, Farallones de Cali Natural National Park, 25. X-8.XI.2000, Malaise trap, S. Sarria (1 female, USNMENT00977899 (USNM)). Vaupés Dept., environmental center, M.3620, 60m, $01^{\circ} 04^{\prime} \mathrm{S} 69^{\circ} 31^{\prime} \mathrm{W}$, Mosiro Itajura-Caparú Biological Station, 27.III3.IV.2003, Malaise trap, J. Pinzón (1 female, USNMENT00977878 (USNM)). Vichada Dept., administrative center, M.272, $100 \mathrm{~m}, 05^{\circ} 21^{\prime} \mathrm{N} 67^{\circ} 51^{\prime} \mathrm{W}$, El Tuparro Natural National Park, 19.VI-29. VI.2000, Malaise trap, W. Villalba (1 female, USNMENT00977444 (UCRC)). COSTA RICA: Puntarenas Prov., Osa Peninsula, house of C. L. Madrigal, Puerto Jiménez, 10m, I-1991, Malaise trap, USNMENT00977843 (deposited in USNM). Puntarenas Prov., Osa Peninsula, house of C. L. Madrigal, Puerto Jiménez, 10m, I-1991, Malaise trap (6 females, 1 male, USNMENT00977832, 00977835, 00977837, 00977839, 00977841, 00977847-00977848 (USNM)). Guanacaste Prov., Filadelfia, 40m, V-1989 - VI-1989 (1 male, USNMENT00977076 (USNM)). Heredia Prov., 3km S Puerto Viejo, Organization for Tropical Studies (OTS), La
Selva Biological Station, 100m, XII-1992, P. Hanson (1 female, USNMENT00977964 (USNM)). Heredia Prov., Huertos, La Selva Biological Station, X-1992, P. Hanson (1 female, USNMENT00977961 (USNM)). Heredia Prov., Organization for Tropical Studies (OTS), virgin forest, $75 \mathrm{~m}, 10^{\circ} 26^{\prime} \mathrm{N}$ $84^{\circ} 01^{\prime} \mathrm{W}$, La Selva Biological Station, XII-1992, Malaise trap (1 female, USNMENT00977811 (USNM)). Puntarenas Prov., 3km SW Rincón, VI-1991 (1 female, USNMENT00977082 (USNM)). Puntarenas Prov., 3km SW Rincón, forest edge, Golfo Dulce Forest Reserve, 10m, III-1991 VI-1991, Malaise trap, M. Pérez Parra (1 female, USNMENT00977791 (USNM)). Puntarenas Prov., 3km SW Rincón, forest edge, Golfo Dulce Forest Reserve, 10m, IX-1991, Malaise trap, M. Pérez Parra (1 female, USNMENT00977795 (USNM)). Puntarenas Prov., 3km SW Rincón, forest edge, Golfo Dulce Forest Reserve, 10m, VI-1991, Malaise trap, M. Pérez Parra (1 female, USNMENT00977792 (USNM)). Puntarenas Prov., 3km SW Rincón, forest edge, Golfo Dulce Forest Reserve, 10m, VIII-1991, Malaise trap, M. Pérez Parra (1 female, USNMENT00977793 (USNM)). Puntarenas Prov., 3km SW Rincón, forest edge, Golfo Dulce Forest Reserve, 10m, X-1991, Malaise trap, M. Pérez Parra (1 male, USNMENT00977794 (USNM)). Puntarenas Prov., 5km W Piedras Blancas, XI-1990 (2 females, USNMENT0097708500977086 (USNM)). Puntarenas Prov., 5km W Piedras Blancas, $08^{\circ} 46^{\prime} \mathrm{N} 83^{\circ} 17^{\prime} \mathrm{W}$, Golfo Dulce Forest Reserve, 1990 (2 females, USNMENT00977073-00977074 (USNM)). Puntarenas Prov., 5km W Piedras Blancas, $2^{\circ}$ forest edge, $100 \mathrm{~m}, 08^{\circ} 46^{\prime} \mathrm{N}$ $83^{\circ} 17^{\prime} \mathrm{W}$, Golfo Dulce Forest Reserve, I-1991 - III-1991, Malaise trap, S. Ramos (1 female, USNMENT00977797 (USNM)). Puntarenas Prov., 5km W Piedras Blancas, $2^{\circ}$ forest edge, $100 \mathrm{~m}, 08^{\circ} 46^{\prime} \mathrm{N} 83^{\circ} 17^{\prime} \mathrm{W}$, Golfo Dulce Forest Reserve, IV-1992 V-1992, Malaise trap, S. Ramos (1 male, 
USNMENT00977796 (USNM)). Puntarenas Prov., 5km W Piedras Blancas, $2^{\circ}$ forest edge, $100 \mathrm{~m}, 08^{\circ} 46^{\prime} \mathrm{N} 83^{\circ} 17^{\prime} \mathrm{W}$, Golfo Dulce Forest Reserve, IV-1993 V-1993, Malaise trap, S. Ramos (1 female, USNMENT00977799 (USNM)). Puntarenas Prov., $5 \mathrm{~km}$ W Piedras Blancas, $2^{\circ}$ forest edge, $100 \mathrm{~m}, 08^{\circ} 46^{\prime} \mathrm{N} 83^{\circ} 17^{\prime} \mathrm{W}$, Golfo Dulce Forest Reserve, VI-1993 - VII1993, Malaise trap, S. Ramos (1 female, USNMENT00977800 (USNM)). Puntarenas Prov., $5 \mathrm{~km} \mathrm{~W}$ Piedras Blancas, $2^{\circ}$ forest edge, $100 \mathrm{~m}, 08^{\circ} 46^{\prime} \mathrm{N} 83^{\circ} 17^{\prime} \mathrm{W}$, Golfo Dulce Forest Reserve, VIII-1993 IX-1993, Malaise trap, S. Ramos (3 females, USNMENT00977807-00977808, 00977960 (USNM)). Puntarenas Prov., 5km W Piedras Blancas, $2^{\circ}$ forest edge, $100 \mathrm{~m}$, $08^{\circ} 46^{\prime} \mathrm{N} 83^{\circ} 17^{\prime} \mathrm{W}$, Golfo Dulce Forest Reserve, X-1990, Malaise trap, S. Ramos (1 female, USNMENT00977798 (USNM)). Puntarenas Prov., 5km W Piedras Blancas, $2^{\circ}$ forest edge, $100 \mathrm{~m}, 08^{\circ} 46^{\prime} \mathrm{N} 83^{\circ} 17^{\prime} \mathrm{W}$, Golfo Dulce Forest Reserve, X-1991, Malaise trap, S. Ramos (3 females, USNMENT00977803-00977805 (USNM)). Puntarenas Prov., $5 \mathrm{~km}$ W Piedras Blancas, $2^{\circ}$ forest edge, $100 \mathrm{~m}, 08^{\circ} 46^{\prime} \mathrm{N} 83^{\circ} 17^{\prime} \mathrm{W}$, Golfo Dulce Forest Reserve, X-1992, Malaise trap, S. Ramos (1 female, USNMENT00977806 (USNM)). Puntarenas Prov., $5 \mathrm{~km} \mathrm{~W}$ Piedras Blancas, $2^{\circ}$ forest edge, $100 \mathrm{~m}, 08^{\circ} 46^{\prime} \mathrm{N} 83^{\circ} 17^{\prime} \mathrm{W}$, Golfo Dulce Forest Reserve, XI-1991 - XII-1991, Malaise trap, S. Ramos (2 females, USNMENT00977801-00977802 (USNM)). Puntarenas Prov., 5km W Piedras Blancas, $2^{\circ}$ forest edge, $100 \mathrm{~m}, 08^{\circ} 46^{\prime} \mathrm{N} 83^{\circ} 17^{\prime} \mathrm{W}$, Golfo Dulce Forest Reserve, XII-1990, Malaise trap, S. Ramos (1 female, USNMENT00977763 (USNM)). Puntarenas Prov., 5km W Piedras Blancas, Golfo Dulce Forest Reserve, 100m, X-1992, P. Hanson (1 female, USNMENT00977959 (USNM)). Puntarenas Prov., 9km SW Rincón, III-1992 - IV-1992 (1 female, USNMENT00977083 (USNM)). Puntarenas Prov., La Sirena,
Corcovado National Park, IV-1989 - VIII1989 (1 female, USNMENT00977078 (USNM)). Puntarenas Prov., Osa Peninsula, house of C. L. Madrigal, Puerto Jiménez, 10m, I-1991, Malaise trap (2 females, USNMENT00977758-00977759 (USNM)). Puntarenas Prov., Osa Peninsula, house of C. L. Madrigal, Puerto Jiménez, 10m, XI-1990, Malaise trap (1 female, USNMENT00977962 (USNM)). Puntarenas Prov., Osa Peninsula, house of C. L. Madrigal, Puerto Jiménez, 10m, XII1990, Malaise trap (1 female, USNMENT00977963 (USNM)). Puntarenas Prov., Puerto Jiménez, I-1991 (2 females, 2 males, USNMENT00977071, 00977729, 00977731, 00977733 (USNM)). Puntarenas Prov., Puerto Jiménez, X-1990 (1 female, USNMENT00977069 (USNM)). Puntarenas Prov., Puerto Jiménez, 10m, XI-1990 (1 male, USNMENT00977070 (USNM)). Puntarenas Prov., Sirena Station, Corcovado National Park, 50m, X-1990 XII-1990, Malaise trap, P. Hanson (1 female, USNMENT00977965 (USNM)). Rio Frio, XI-1989 (1 female, USNMENT00977075 (USNM)). San José Prov., San Isidro (San Isidro del General), 22. VII.1981, N. L. H. Krauss (1 male, USNMENT00977445 (UCRC)). ECUADOR: Napo Prov., 10km NE Tena, 400m, 19.II20.II.1983, L. Masner (4 females, USNMENT00977689, 00977699-00977701 (USNM)). Pichincha Prov., 47km S Santo Domingo de los Colorados, 22.VII-28. VII.1976, Malaise trap, J. Cohen (1 female, USNMENT00977666 (USNM)). Pichincha Prov., E of Puerto Quito, 2. II.1983, L. Huggert (1 female, USNMENT00977706 (USNM)). Santo Domingo de los Tsáchilas Prov., Tinalandia, 800m, 2.II.1983, Masner \& Sharkey (1 female, USNMENT00993693 (USNM)). PANAMA: Bocas del Toro Prov., $8.8 \mathrm{~km}$ W Rambaia, 2001/028, 35m, 08 $59^{\prime} 04^{\prime \prime} \mathrm{N}$ $82^{\circ} 13^{\prime} 57^{\prime \prime} \mathrm{W}$, La Gloria River, 8.I.2001, screen sweeping, M. Yoder \& J. B. 

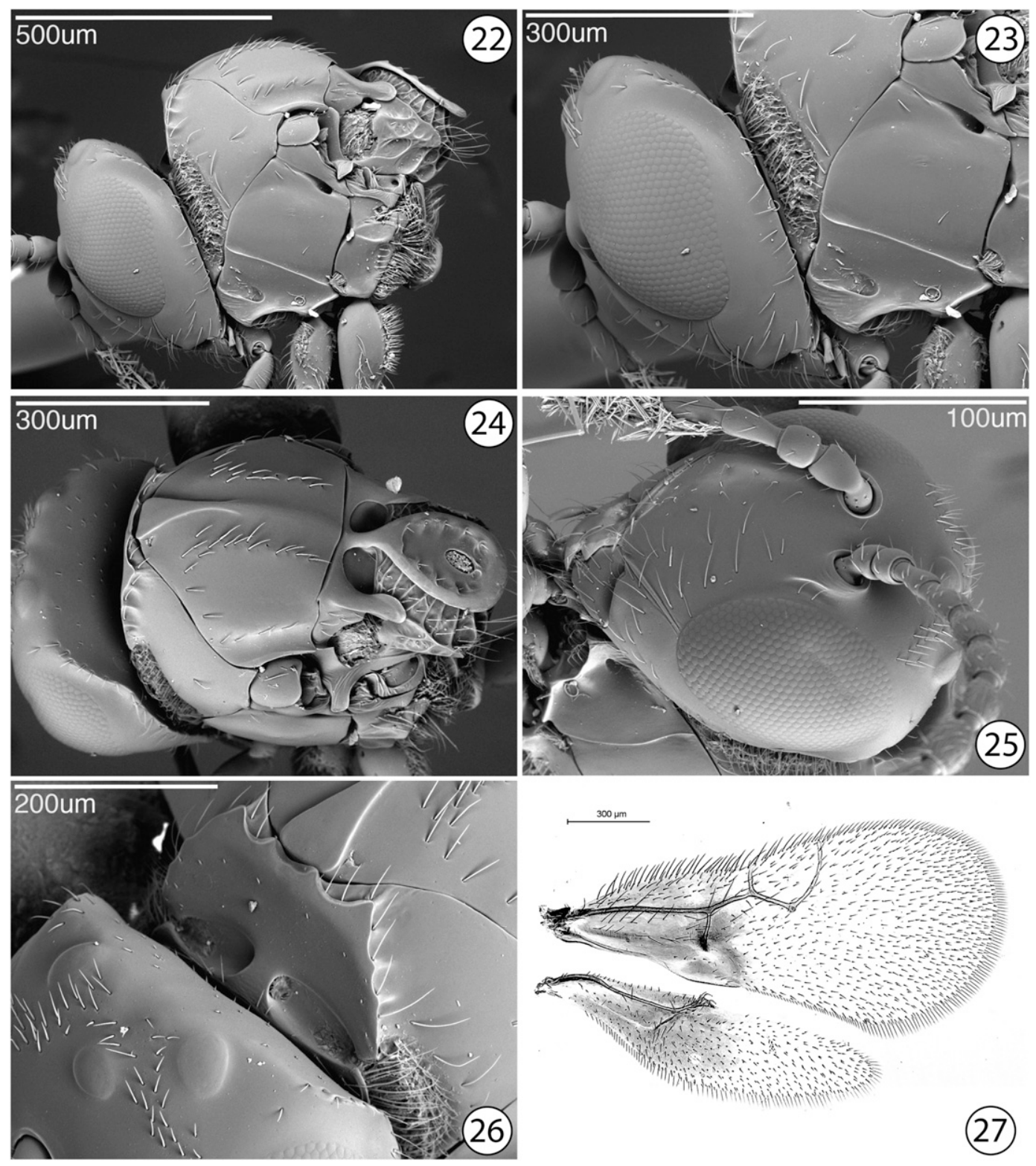

Plate 6, Figs. 22-27. Zaeucoila grenadensis (Ashmead). 22, head and mesosoma, lateral view; 23, head and mesopleuron, lateral view; 24, head and mesosoma, dorsal view; 25, head, anterior view; 26, pronotal plate, antero-dorsal view; 27, fore and hind wings.

Woolley (1 female, USNMENT00977668 (USNM)). Chiriquí Prov., 5km SW Volcán, 99/069, 4220ft, $08^{\circ} 45^{\prime \prime} 52^{\prime \prime} \mathrm{N} 82^{\circ} 40^{\prime} 33^{\prime \prime} \mathrm{W}$, Volcán Lagoon, 30.VII.1999, J. B. Woolley (1 female, USNMENT00977399
(TAMU)). PERU: Madre de Dios Reg., Manu Prov., Pillcopata, secondary forest, H11-157, 526m, 12 $53^{\prime} 07^{\prime \prime S} 71^{\circ} 23^{\prime} 48^{\prime \prime} \mathrm{W}$, Villa Carmen Research Station, 27. XI.2011, sweeping, J. Heraty (1 male, 
USNMENT00993870 (USNM)). TRINIDAD AND TOBAGO: Diego Martin Reg., Trinidad Isl., Moka Estate, II-1977, Malaise trap, C. Lawrence (1 female, USNMENT00969612 (BMNH)). Mayaro/Rio Claro Reg., Trinidad Isl., cocoa plantation, Ecclesville, 28.VII.1976, J. S. Noyes (1 female, USNMENT00969678 (BMNH)). Tobago Isl., neglected citrus orchard next to primary forest, Goldsborough, 17.III-24. III.1994, Malaise trap, M. J. Sommeijer (1 female, USNMENT01223591 (USNM)). Trinidad Isl., Maracas Valley, X-1977, Malaise trap, F. D. Bennett (1 female, USNMENT00969600 (BMNH)). Tunapuna/ Piarco Reg., Trinidad Isl., Curepe, 17. XII.1978 (1 female, USNMENT00977429 (UCRC)). Tunapuna/Piarco Reg., Trinidad Is1., Saint Augustine, 28065, Malaise trap, F. D. Bennett (1 female, USNMENT00969597 (BMNH)). Tunapuna/ Piarco Reg., Trinidad Isl., Saint Augustine, IX-1976, Malaise trap, F. D. Bennett (3 females, USNMENT00969533, 00969731, 00969743 (BMNH)). VENEZUELA: Sucre St., 7km NW San Vicente, 23.III.1982, G. F. Hevel \& J. F. Hevel (1 female, USNMENT00993988 (USNM)).

Distribution.-Neotropical Region: Argentina, Bolivia, Colombia, Costa Rica, Ecuador, Grenada, Panama, Peru, Trinidad and Tobago and Venezuela.

Biology.-Host not observed.

\section{Zaeucoila grenadensis (Ashmead, 1900), new combination}

http://bioguid.osu.edu/xbiod_concepts/ 407941

Figures 22-27

Diranchis grenadensis Ashmead, 1900: 248 (original description).

Moneucoela grenadensis (Ashmead): Dalla Torre and Kieffer, 1910: 103 (generic transfer).

Aegeseucoela grenadensis (Ashmead): Buffington, 2002: 595 (generic transfer).
Agrostocynips grenadensis (Ashmead): Buffington, 2009: 172 (generic transfer, listed).

Redescription.-Coloration with head, mesosoma, metasoma black to dark brown; legs with coxae and femora dark brown, rest yellow. Lateral margin of occiput defined by evenly rounded, raised, sharp carina. Vertical carina adjacent to ventral margin of antennal socket present, leading to shallow inner orbital groove. Malar space adjacent to anterior articulation of mandible with short, blunt, smooth protuberance. Malar sulcus present, composed of single line. Orbital furrows rounded, ill defined, running from lateral edge of torulus to middle of compound eye. Dorsal aspect of vertex smooth with a few short, scattered setae. Posterior aspect of vertex smooth with fine setae. Hair punctures on lateral aspect of vertex present, indistinct. Placoidal sensilla present on F3-11. Pubescence on lateral surface of pronotum present, sparse, with distinct row behind pronotal plate. Ridges extending posteriorly from lateral margin of pronotal plate present, variable, short to medium long, in some cases running to anterior margin of mesoscutum. Sculpture on mesoscutum absent, with medium density setal tracks along anterior margin, following parallel to mesoscutal carina. Median mesoscutal carina present, composed of anteriorly broad elevation, narrowing posteriorly, typically ending at midline of mesoscutum, but with distinct burm continuing to posterior margin of mesoscutum. Anterior admedial lines present, in conjunction with mesoscutal carina. Lateroventral mesopleural carina present, marking abrupt change of slope of mesopectus, terminating anteriorly just ventral to large, oblique notch. Mesopleural triangle present, gently impressed with distinct dorsal border, ventral border lacking; smooth. Dorsal surface of scutellum laterally with distinct ridges 
radiating from plate, posteriorly with deep fovea and ridges. Latero-ventral margin of scutellum posterior to auricula deeply foveate. Subalar area abruptly broadened anteriorly, with an indicated longitudinal division. Pubescence present, but very sparse, along posterior and ventral margins of metapleuron; short and dense on propodeum. Pubescence of fore wing mesally sparse, gradually becoming denser distally. Coloration of wing basally infuscate, apically hyaline. Hair fringe along apical margin of fore wing present, with particularly longer setae along the mesal anterior margin. Inter propodeal carinae space moderately setose, with long, thin setae evenly filling the space; surface underneath smooth.

Diagnosis. - The only Zaeucoila with distinct ocellar hair patches present, as well as complete parapsidal hair lines.

Link to Distribution Map.-[http:// hol.osu.edu/map-full.html id=407941]

Material Examined.-Holotype: Grenada, West Indies. Balthazar (windward side), H.H. Smith (Coll.), NHM (\#7.50); the type specimen is in poor condition, with the head plus thorax on one end of a pinned card, and the metasoma on the other end. Other material: (153 females, 86 males) ARGENTINA: Buenos Aires Prov., 34³5'06"S 5904'45"W, Luján, 31. III.2005, Malaise trap, Coviella (1 male, USNMENT01022002 (USNM)). Buenos Aires Prov., $34^{\circ} 35^{\prime} 06^{\prime \prime} \mathrm{S} 5^{\circ} 04^{\prime} 45^{\prime \prime} \mathrm{W}$, Luján, 31.III.2005, Malaise trap (1 male, USNMENT01022115 (USNM)). BELIZE: Cayo Dist., Millionario-Resumadero Rd., Chiquibul Forest Reserve, 11.II.2000, reared, O. T. Lewis (1 male, USNMENT00977205 (USNM)). Cayo Dist., Millionario-Resumadero Rd., Chiquibul Forest Reserve, 12.II.2000, reared, O. T. Lewis (2 females, USNMENT00977203-00977204 (USNM)). Cayo Dist., Millionario-Resumadero Rd., Chiquibul Forest Reserve, 14.II.2000, reared, O. T. Lewis (4 females, 2 males, USNMENT00977148-00977153 (USNM)). Cayo Dist., Millionario-Resumadero Rd., Chiquibul Forest Reserve, 24.III.2000, reared, O. T. Lewis (1 female, USNMENT00977206 (USNM)). Cayo Dist., Millionario-Resumadero Rd., Chiquibul Forest Reserve, 25.II.2000, reared, O. T. Lewis (4 females, 3 males, USNMENT00977192-00977193, 0097719500977199 (USNM)). Cayo Dist., MillionarioResumadero Rd., Chiquibul Forest Reserve, 26.II.2000, reared, O. T. Lewis (1 female, 1 male, USNMENT0097718600977187 (USNM)). Cayo Dist., Millionario-Resumadero Rd., Chiquibul Forest Reserve, 30.I.2000, reared, O. T. Lewis (3 females, 1 male, USNMENT0097718800977191 (USNM)). Cayo Dist., Millionario-Resumadero Rd., Chiquibul Forest Reserve, 7.II.2000, reared, O. T. Lewis (10 females, 8 males, USNMENT0097716700977173, 00977175-00977185 (USNM)). Cayo Dist., Millionario-Resumadero Rd., Chiquibul Forest Reserve, 8.II.2000, reared, O. T. Lewis (4 females, 2 males, USNMENT00977154-00977159 (USNM)). Cayo Dist., Millionario-Resumadero Rd., Chiquibul Forest Reserve, 9.II.2000, reared, O. T. Lewis ( 2 females, 1 male, USNMENT00977200-00977202 (USNM)). Cayo Dist., Monkey Tail Track, Chiquibul Forest Reserve, 27.I.2000, reared, O. T. Lewis (1 female, USNMENT00977208 (USNM)). Cayo Dist., Monkey Tail Track, Chiquibul Forest Reserve, 28.I.2000, reared, O. T. Lewis (1 male, USNMENT00977207 (USNM)). Cayo Dist., Monkey Tail Track, Chiquibul Forest Reserve, III-2000, reared, O. T. Lewis ( 2 females, 5 males, USNMENT00977160-00977166 (USNM)). BOLIVIA: El Beni Dept., Estancia El Porvenir, 40km E San Borja, lake shore / open grass savanna, Beni Biological Station, 6.IX-8.IX.1987, Malaise trap, W. E. Steiner (1 male, USNMENT01022001 (USNM)). Santa Cruz Dept., 370m, $17^{\circ} 40^{\prime} \mathrm{S} 63^{\circ} 27^{\prime} \mathrm{W}$, 
Potrerillos del Guenda Nature Reserve, 20.X-25.X.2007, Malaise trap, J. Romero (2 females, USNMENT01022003-01022004 (USNM)). BRAZIL: GO, Itumbiara, 11.IV.2002, Marchiori (1 female, 1 male, USNMENT01197596-01197597 (MLPA)). GO, Itumbiara, 18.IV.2002, Marchiori (4 females, 1 male, USNMENT01197598-01197602 (MLPA)). GO, Itumbiara, 19.II-23.II.1996, Malaise trap ( 1 female, 1 male, USNMENT01197612-01197613 (MZSP)). GO, Itumbiara, 2.V.2002, Marchiori (1 female, USNMENT01197606 (MLPA)). GO, Itumbiara, 2.VI.1998, Marchiori (1 female, USNMENT01197595 (MLPA)). GO, Itumbiara, 20.II-25.II.1996, Malaise trap (1 female, USNMENT01197614 (MZSP)). GO, Itumbiara, 25.IV.2002, Marchiori (2 females, 1 male, USNMENT01197603-01197605 (MLPA)). GO, Itumbiara, 5.X.1995, yellow pan trap (2 females, 2 males, USNMENT0119760801197611 (MZSP)). GO, Itumbiara, 9. V.2002, Marchiori (1 female, USNMENT01197607 (MLPA)). MG, Araporã, 11.IV.2002, Marchiori (1 male, USNMENT01197615 (MLPA)). MG, Araporã, 18.IV.2002, Marchiori (2 males, USNMENT01197616-01197617 (MLPA)). MG, Araporã, 2.V.2002, Marchiori (1 male, USNMENT01197618 (MLPA)). MG, Araporã, 20.VI.2002, Marchiori (1 female, USNMENT01197621 (MLPA)). MG, Araporã, 26.IX.2002, Marchiori (1 male, USNMENT01197624 (MLPA)). MG, Araporã, 4.VII.2002, Marchiori (2 males, USNMENT01197622-01197623 (MLPA)). MG, Araporã, 6.VI.2002, Marchiori (2 females, USNMENT01197619-01197620 (MLPA)). RJ, Silva Jardim Mpio., Poço das Antas Biological Reserve, 1998, reared, Barbosa da Silva (17 females, 9 males, USNMENT01197625-01197650 (MLPA)). COLOMBIA: Bolívar Dept., La Yaya, M.2632, 280m, $09^{\circ} 54^{\prime} \mathrm{N} 75^{\circ} 07^{\prime} \mathrm{W}$, Los Colorados Fauna and Flora Sanctuary, 14.
XI-30.XI.2001, Malaise trap, E. Deulufeut (1 female, USNMENT01022068 (USNM)). montane rainforest, $500 \mathrm{~m}, 05^{\circ} 45^{\prime} \mathrm{N}$ $76^{\circ} 30^{\prime} \mathrm{W}$, Chocó Dept., 11.IV-13.IV. 1973 (1 female, USNMENT00977618 (USNM)). COSTA RICA: Guanacaste Prov., Coopemarti, IX-1990 (1 female, USNMENT00977081 (USNM)). Guanacaste Prov., Coopemarti, X-1990 (2 females, USNMENT00977079-00977080 (USNM)). Guanacaste Prov., H.1.O, Santa Rosa National Park, 300m, 14.VIII-6. IX.1986, Malaise trap, D. Janzen \& I. Gauld (1 female, USNMENT01022060 (USNM)). Guanacaste Prov., H.1.O, Santa Rosa National Park, 300m, 27.IV-11. V.1985, Malaise trap, D. Janzen \& I. Gauld (1 male, USNMENT01022089 (USNM)). Guanacaste Prov., H.1.O, Santa Rosa National Park, 300m, 5.VII-26.VII. 1986, Malaise trap, D. Janzen \& I. Gauld (1 male, USNMENT01022053 (USNM)). Guanacaste Prov., H.1.O, Santa Rosa National Park, 300m, 6.IX-27.IX.1986, Malaise trap, D. Janzen \& I. Gauld (2 females, USNMENT01022091-01022092 (USNM)). Guanacaste Prov., H.2.C, Santa Rosa National Park, 300m, 14.III-4. IV.1987, Malaise trap, D. Janzen \& I. Gauld (1 female, USNMENT01022064 (USNM)). Guanacaste Prov., H.2.C, Santa Rosa National Park, 300m, 14.VI-5. VII.1986, Malaise trap, D. Janzen \& I. Gauld (1 male, USNMENT01022063 (USNM)). Guanacaste Prov., H.2.C, Santa Rosa National Park, 300m, 20.XII-10. I.1987, Malaise trap, D. Janzen \& I. Gauld (1 male, USNMENT01022065 (USNM)). Guanacaste Prov., H.2.C, Santa Rosa National Park, 300m, 21.II-14.III.1987, Malaise trap, D. Janzen \& I. Gauld (1 female, USNMENT01022058 (USNM)). Guanacaste Prov., H.2.C, Santa Rosa National Park, 300m, 22.VI-13.VII.1985, Malaise trap, D. Janzen \& I. Gauld (1 female, USNMENT01022070 (USNM)). Guanacaste Prov., H.2.C, Santa Rosa National 
Park, 300m, 23.III.1986, Malaise trap, D. Janzen \& I. Gauld (1 female, USNMENT01022061 (USNM)). Guanacaste Prov., H.2.C, Santa Rosa National Park, 300m, 24.V-13.VI.1982, Malaise trap, D. Janzen \& I. Gauld (1 female, USNMENT01022084 (USNM)). Guanacaste Prov., H.2.C, Santa Rosa National Park, 300m, 26.VII-14.VIII.1986, Malaise trap, D. Janzen \& I. Gauld (1 female, USNMENT01022013 (USNM)). Guanacaste Prov., H.2.C, Santa Rosa National Park, 300m, 6.IX-27.IX.1986, Malaise trap, D. Janzen \& I. Gauld (1 female, USNMENT01022059 (USNM)). Guanacaste Prov., H.2.C, Santa Rosa National Park, 300m, 8.II-2.III.1986, Malaise trap, D. Janzen \& I. Gauld (1 female, USNMENT01022062 (USNM)). Guanacaste Prov., H.3.O, Santa Rosa National Park, 300m, 11.V-1.VI.1985, Malaise trap, D. Janzen \& I. Gauld (5 females, 3 males, USNMENT01022041-01022046, 01022066-01022067 (USNM)). Guanacaste Prov., H.3.O, Santa Rosa National Park, 300m, 13.VII-3.VIII.1985, Malaise trap, D. Janzen \& I. Gauld (2 females, USNMENT01029098 (NMNH); USNMENT01022047 (USNM)). Guanacaste Prov., H.3.O, Santa Rosa National Park, 300m, 14.III-4.IV.1987, Malaise trap, D. Janzen \& I. Gauld (1 male, USNMENT01022057 (USNM)). Guanacaste Prov., H.3.O, Santa Rosa National Park, 300m, 14.VI-5.VII.1986, Malaise trap, D. Janzen \& I. Gauld (4 females, 13 males, USNMENTO0977632, 0102202101022036 (USNM)). Guanacaste Prov., H.3.O, Santa Rosa National Park, 300m, 14.VIII-6.IX.1985, Malaise trap, D. Janzen \& I. Gauld (4 females, 1 male, USNMENT01022071, 01022073, 0102207601022078 (USNM)). Guanacaste Prov., H.3.O, Santa Rosa National Park, 300m, 2. III-23.III.1986, Malaise trap, D. Janzen \& I. Gauld (1 female, USNMENT01022090 (USNM)). Guanacaste Prov., H.3.O, Santa
Rosa National Park, 300m, 20.XII.1986, Malaise trap, D. Janzen \& I. Gauld (1 male, USNMENT01022074 (USNM)). Guanacaste Prov., H.3.O, Santa Rosa National Park, 300m, 21.II-14.III.1987, Malaise trap, D. Janzen \& I. Gauld (1 female, USNMENT01022048 (USNM)). Guanacaste Prov., H.3.O, Santa Rosa National Park, 300m, 23.VI-13.VII.1985, Malaise trap, D. Janzen \& I. Gauld (1 female, 2 males, USNMENT01022079-01022081

(USNM)). Guanacaste Prov., H.3.O, Santa Rosa National Park, 300m, 26.VII-14. VIII.1986, Malaise trap, D. Janzen \& I. Gauld (1 female, USNMENT01022094 (USNM)). Guanacaste Prov., H.3.O, Santa Rosa National Park, 300m, 27.IX-18. X.1986, Malaise trap, D. Janzen \& I. Gauld (6 females, USNMENT0102201801022020, 01022085-01022087 (USNM)). Guanacaste Prov., H.3.O, Santa Rosa National Park, 300m, 4.V-24.V.1986, Malaise trap, D. Janzen \& I. Gauld (1 female, USNMENT01022056 (USNM)). Guanacaste Prov., H.3.O, Santa Rosa National Park, 300m, 5.VII-26.VII.1986, Malaise trap, D. Janzen \& I. Gauld (2 females, 4 males, USNMENT01022014-01022017, 01022082-01022083 (USNM)). Guanacaste Prov., H.3.O, Santa Rosa National Park, 300m, 6.IX-27.IX.1986, Malaise trap, D. Janzen \& I. Gauld (1 female, 2 males, USNMENT01022050-01022052 (USNM)). Guanacaste Prov., H.4.C, Santa Rosa National Park, 300m, 22.VI-13. VII.1985, Malaise trap, D. Janzen \& I. Gauld (1 female, USNMENT01022055 (USNM)). Guanacaste Prov., H.4.C, Santa Rosa National Park, 300m, 24.V-14. VI.1986, Malaise trap, D. Janzen \& I. Gauld (2 females, USNMENT01022072, 01022075 (USNM)). Guanacaste Prov., H.4.C, Santa Rosa National Park, 300m, 27.IV-11.V.1985, Malaise trap, D. Janzen \& I. Gauld (1 female, USNMENT01022049 (USNM)). Guanacaste Prov., H.4.C, Santa Rosa National Park, 
300m, 4.V-24.V.1986, Malaise trap, D. Janzen \& I. Gauld (1 female, USNMENT01022054 (USNM)). Guanacaste Prov., Osa Peninsula, 8km S bridge, Coopemarti, 30m, XII-1990 (2 females, USNMENT01022005, 01022037 (USNM)). Guanacaste Prov., SE.7.O1, Santa Rosa National Park, 300m, 1.V-22.V.1985, Malaise trap, D. Janzen \& I. Gauld (1 female, USNMENT01022093 (USNM)). Guanacaste Prov., SE.8.C, Santa Rosa National Park, 300m, 23.III-13.IV.1986, Malaise trap, D. Janzen \& I. Gauld (1 female, USNMENT01022088 (USNM)). Heredia Prov., Chilamate, 75m, 25.III.1989, Hanson \& Godoy (1 female, USNMENT01022069 (USNM)). Heredia Prov., La Selva Biological Station, 50-150m, VIII-1992 (1 female, USNMENT00977077 (USNM)). Heredia Prov., Organization for Tropical Studies (OTS), virgin forest, Huertos, La Selva Biological Station, 75m, IX-1992, Malaise trap (1 female, USNMENT01022009 (USNM)). Heredia Prov., Organization for Tropical Studies (OTS), virgin forest, Huertos, La Selva Biological Station, 75m, VIII-1992, Malaise trap (1 female, USNMENT01022038 (USNM)). Puntarenas Prov., 5km N Puerto Jiménez, IX-1992 - X-1992 (1 female, USNMENT00977063 (USNM)). Puntarenas Prov., Osa Peninsula, house of C. L. Madrigal, Puerto Jiménez, 10m, II-1991, Malaise trap (2 females, 1 male, USNMENT00977750, 00977753, 01022006 (USNM)). Puntarenas Prov., Osa Peninsula, house of C. L. Madrigal, Puerto Jiménez, 10m, III-1991, Malaise trap (3 females, USNMENT00977749, 0102203901022040 (USNM)). Puntarenas Prov., Osa Peninsula, house of C. L. Madrigal, Puerto Jiménez, 10m, III-1992, Malaise trap (2 females, USNMENT01022011-01022012 (USNM)). Puntarenas Prov., Osa Peninsula, house of C. L. Madrigal, Puerto Jiménez, 10m, VIII-1991, Malaise trap (1 female, USNMENT01022010 (USNM)). Puntarenas
Prov., Osa Peninsula, house of C. L. Madrigal, Puerto Jiménez, 10m, X1991, Malaise trap (2 females, USNMENT01022007-01022008 (USNM)). Puntarenas Prov., Puerto Jiménez, I-1991 (1 female, USNMENT00977064 (USNM)). Puntarenas Prov., Puerto Jiménez, IV1991 (2 females, USNMENT00977067, 00977072 (USNM)). Puntarenas Prov., Puerto Jiménez, X-1990 (1 female, USNMENT00977065 (USNM)). Puntarenas Prov., Puerto Jiménez, XI-1990 (1 female, USNMENT00977068 (USNM)). Puntarenas Prov., Puerto Jiménez, XII-1991 (1 female, USNMENT00977066 (USNM)). San José Prov., Colón (Ciudad Colón), III-1990 - IV-1990 (1 female, USNMENT00977084 (USNM)). CURAÇAO: forest, Christoffel National Park, 150m, 5. XI-17.XI.2007, yellow pan trap, L. Masner (2 females, USNMENT0099383300993834 (USNM)). ECUADOR: Napo Prov., Puerto Misahuallí, 18.II.1983, L. Huggert (2 females, USNMENT00977603, 00977605 (USNM)). MEXICO: TAMPS, 1986/002, 5mi W Gómez Farías, 20. III.1986, Woolley \& Zolnerowich (1 male, USNMENT00977385 (TAMU)). TAMPS, Los Cedros Field Station, 340m, $23^{\circ} 03^{\prime} 00^{\prime \prime} \mathrm{N}$ 99 $09^{\prime} 03^{\prime \prime} \mathrm{W}$, Gómez Farías, 14.IV.2002, Malaise trap, A. CordobaTorres (1 male, USNMENT01022116 (USNM)). PANAMA: Bocas del Toro Prov., 8.8km W Rambaia, La Gloria River, 8.I.2001 (1 female, USNMENT01022000 (USNM)). Panamá Prov., Canal Zone, Summit Park, IX-1946, N. L. H. Krauss (1 female, USNMENT00977572 (USNM)). PERU: Madre de Dios Reg., Tambopata National Reserve, 17.X-1.XI.1983, N. E. Stork (1 male, USNMENT00970040 $(\mathrm{BMNH})$ ). UNKNOWN: no date (1 female, 1 male, USNMENT00977209 (TAMU); USNMENT00977210 (USNM)).

Comments.-Both diagnostic characters (above) are shared with Marthiella. However, the species does not fit 


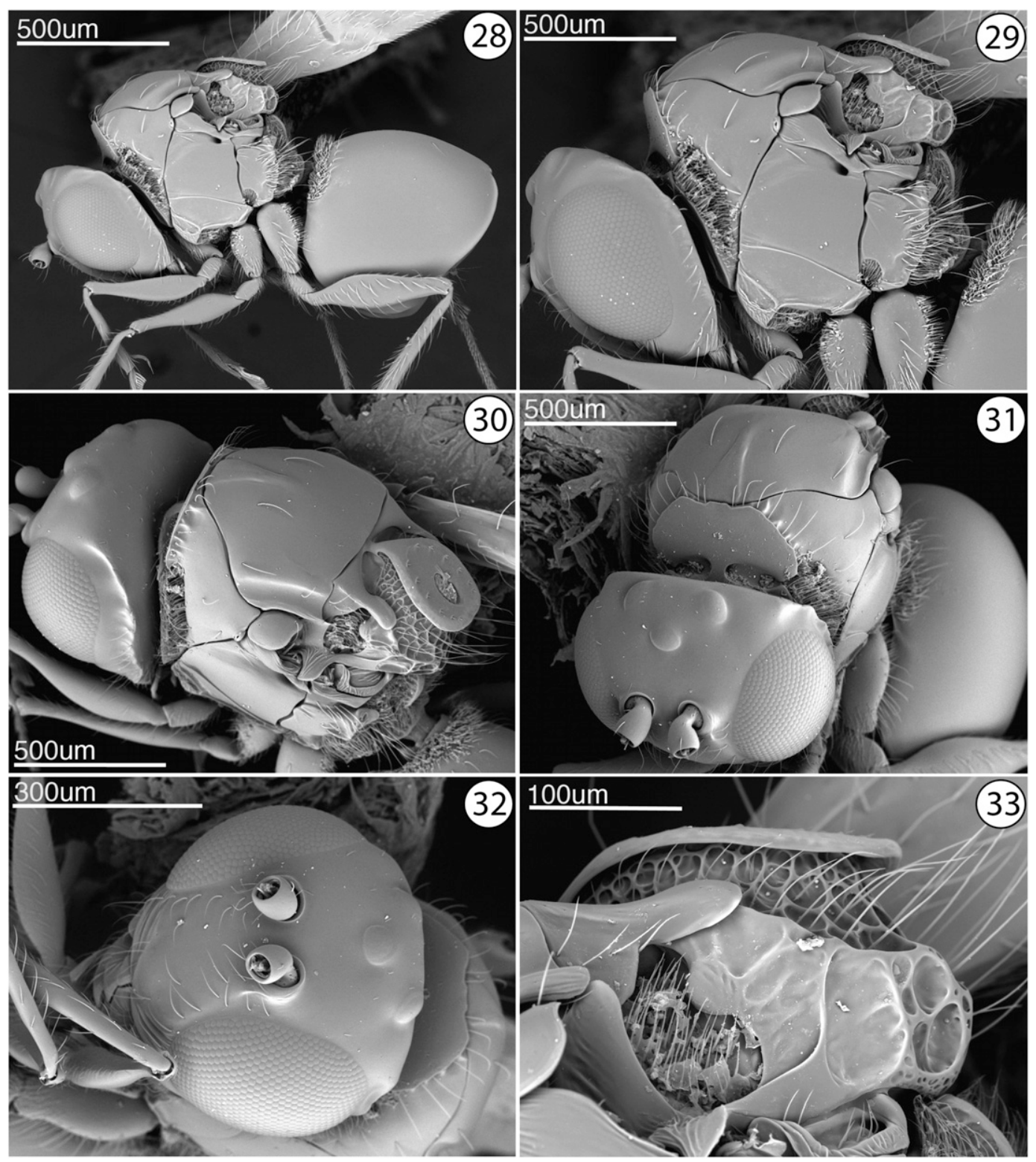

Plate 7. Figs. 28-33. Zaeucoila incompleta (Ashmead). 28, habitus, lateral view; 29, head and mesosoma, lateral view; 30, head and mesosoma, postero-dorsal view; 31, head and mesosoma, anterodorsal view; 32, head, anterior view; 33, scutellum, lateral view.

well into any other genus than that of Zaeucoila. Buffington (2009) recovered this species interspersed among Aegeseucoela, Zaeucoila and Agrostocynips, species of which are all treated here as Zaeucoila.
Distribution.-Neotropical Region: Argentina, Belize, Grenada, Mexico, Panama, Venezuela (Ashmead 1900, Buffington 2002, Salvo 2008). New country record for Brazil, Ecuador and Peru. 

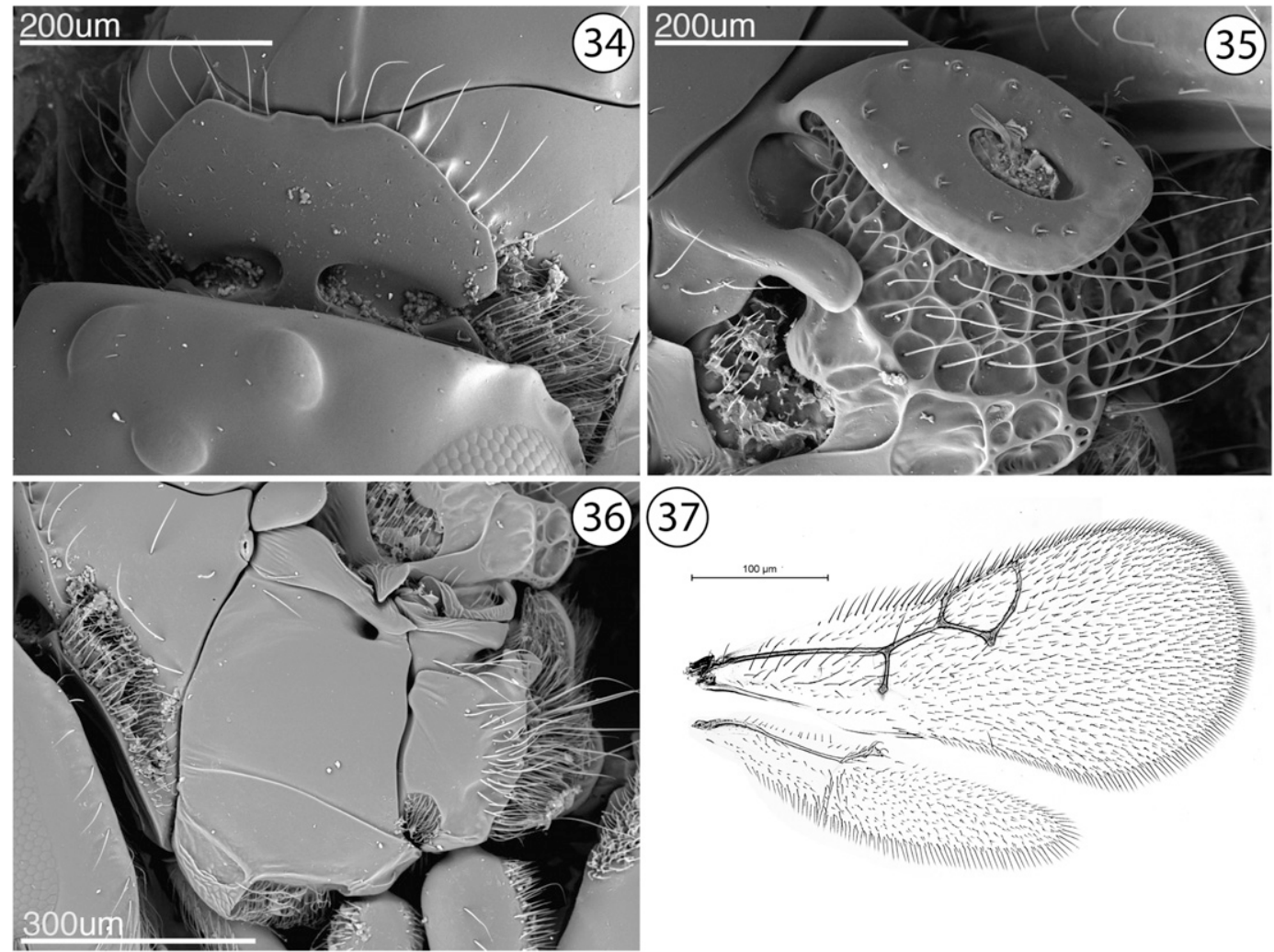

Plate 8, Figs. 34-37. Zaeucoila incompleta (Ashmead). 34, pronotal plate, antero-dorsal view; 35 , scutellum, postero-dorsal view; 36, mesopleuron and metapleuron, lateral view; 37, fore and hind wings.

Biology.-Reared from Diptera Agromyzidae: Calycomyza verbenivora Spencer on Verbena sp. (Verbenaceae), Calycomyza cf. cassiae Frost on Senna cobanensis (Britton \& Rose) (Fabaceae), Haplopeodes sp. on Solanum erianthum D. Don (Solanaceae) (Buffington 2002); Calycomyza hiptidis Spencer, Calycomyza servilis Spencer, Calycomyza eupatorivora Spencer, and an unidentified Calycomyza species are recorded here as new agromyzid host records (based on label data).

\section{Zaeucoila incompleta (Kieffer)}

http://bioguid.osu.edu/xbiod_concepts/ 389225

(Figs. 28-37)
Rhabdeucoela incompleta Kieffer, 1907: 70 (original description, keyed); Kieffer, 1907: 90 (description, distribution).

Zaeucoila incompleta (Kieffer): Weld, 1952: 193 (generic transfer); Díaz and Gallardo, 1997: 37 (description); Buffington, 2009: 185 (listed, host association).

Redescription.-Coloration with head, mesosoma, metasoma black to dark brown; legs variable, ranging from all leg segments entirely orange-yellow to coxae and femora dark brown, rest yellow. Lateral margin of occiput defined by scalloped, sharp carina. Vertical carina adjacent to ventral margin of antennal socket present, leading to deep inner orbital groove. Malar space adjacent to anterior articulation of mandible with 
distinct conical protuberance, smooth. Malar sulcus present, composed of series of small striae. Orbital furrows sharp, divergent groove running from antennal sockets to dorsal end of malar sulcus. Dorsal aspect of vertex smooth with a few short, scattered setae. Posterior aspect of vertex smooth with fine setae. Hair punctures on lateral aspect of vertex absent. Placoidal sensilla present on F 2-11. Pubescence on lateral surface of pronotum present, sparse, with distinct row behind pronotal plate. Ridges extending posteriorly from lateral margin of pronotal plate present, typically short, with single prominent ridge extending to anterior margin of mesoscutum. Sculpture on mesoscutum absent, with sparse long hairs. Median mesoscutal carina present, composed of anteriorly broad elevation, narrowing posteriorly, ending before midline of mesoscutum. Anterior admedial lines present, in conjunction with mesoscutal carina. Lateroventral mesopleural carina present, marking abrupt change of slope of mesopectus, terminating anteriorly at large, oblique notch. Mesopleural triangle present, gently impressed with distinct dorsal border, ventral border lacking; smooth. Dorsal surface of scutellum foveate-areolet. Latero-ventral margin of scutellum posterior to auricula smooth with irregular rugae. Subalar area slightly broadened anteriorly, with an indicated longitudinal division. Pubescence present, medium density, along posterior and ventral margins of metapleuron; short and dense on propodeum. Pubescence of fore wing present, long, dense on most of surface. Coloration of wing absent, entire wing hyaline. Hair fringe along apical margin of fore wing present, with particularly longer setae along the mesal anterior margin. Inter propodeal carinae space moderately setose, with long, thin setae evenly filling the space; surface underneath smooth.

Diagnosis.-This species is most readily confused with $Z$. flavipes, but can be separated from this species by having a smooth genal carina (scalloped in $Z$. flavipes) and lacking a distinct pair of elongate setae on each side of the mesoscutal keel.

Link to Distribution Map.-[http:// hol.osu.edu/map-full.html?id=389225]

Material Examined.-Holotype, female, $R$. incompleta: CUBA: Granma Prov., Cayamas, no date, Baker, CASC Type No. 10551 (deposited in CAS). Other material: (170 females, 135 males, 2 unknowns) ARGENTINA: Buenos Aires Prov., 30km W Buenos Aires, 3438' 57"S 58 46' 57"W, Mariano Moreno, 16.II.2005, Malaise trap, Coviella (1 male, USNMENT00977614 (USNM)). Buenos Aires Prov., 30km W Buenos Aires, 343' $57^{\prime \prime} \mathrm{S} 58^{\circ} 46^{\prime} 57^{\prime \prime} \mathrm{W}$, Mariano Moreno, 3.VII.2005, Malaise trap, Coviella (2 females, USNMENT0099385600993857 (USNM)). Buenos Aires Prov., $30 \mathrm{~km}$ W Buenos Aires, 34 38'57"S 58 46' 57"W, Mariano Moreno, 9.IV.2005, Malaise trap, Coviella (1 male, USNMENT00993765 (USNM)). Buenos Aires Prov., Chascomus, 7.XII.1952, Martin (1 female, USNMENT01197651 (MLPA)). Buenos Aires Prov., N of Buenos Aires, $34^{\circ} 23^{\prime} 50^{\prime \prime} \mathrm{S} 58^{\circ} 34^{\prime} 32^{\prime \prime} \mathrm{W}$, Tigre, 1.IV-14. IV.2006, Malaise trap, Logarzo (1 female, USNMENT01223597 (USNM)). Córdoba Prov., 20.XII-26.XII.1978, Valladares (1 female, USNMENT01197652 (MLPA)). Entre Ríos Prov., 14km SW Concordia, 11.I.1980, C. Vardy \& M. Vardy (1 female, USNMENT00969655 (BMNH)). Formosa Prov., nr. Las Lomitas, acacia scrub / wet chaco, RN 81, 145m, 25.III.2003, sweeping, J. Munro (1 female, USNMENT00977613 (USNM)). La Rioja Prov., El Duraznillo, 1.I-15.I.2003, Malaise trap, P. Fidalgo (2 males, USNMENT00977719-00977720 
(USNM)). La Rioja Prov., Santa Cruz, 15. IX-14.X.2002, Malaise trap, Fidalgo (1 female, USNMENT00977977 (USNM)). La Rioja Prov., Santa Cruz, 31.XII.2002, pan trap, P. Fidalgo (1 female, 10 males, USNMENT00977707-00977715, 00977717-00977718 (USNM)). Misiones Prov., 400m, 2558.471's 5406.986' W, Vida Silvestre "Urugua-í" Wildlife Reserve, 7.XII-9.XII.2003, Malaise trap, B. V. Brown \& G. Kung (1 female, USNMENT00977724 (USNM)). Misiones Prov., Iguazú National Park, 〜40m, 8.IV-11.IV.1974, Malaise trap, C. Vardy \& M. Vardy (5 males, USNMENT00969630, 00969642-00969643, 00969649-00969650 (BMNH)). Misiones Prov., Jesuit Ruins of Loreto, $179 \mathrm{~m}, 27$. III.2003, sweeping, J. Munro (1 male, USNMENT00977722 (USNM)). Misiones Prov., Loreto, X-1930, Ogloblin (1 male, USNMENT01197653 (MLPA)). Misiones Prov., Posadas, V1961 , N. L. H. Krauss (1 female, USNMENT00993937 (USNM)). Misiones Prov., Yerbal Viejo, 16.IV.1930, Ogloblin (1 male, USNMENT01197654 (MLPA)). Misiones Prov., nr. Loreto, S of Santa Ana, 196m, 28.III.2003, sweeping, J. Munro (1 female, USNMENT00977723 (USNM)). Misiones Prov., nr. Loreto, wet forest, $77 \mathrm{~m}, 27.34^{\circ} \mathrm{S} 55.53^{\circ} \mathrm{W}$, Santa Ana, 27.III.2003, J. Munro (1 female, USNMENT00993768 (USNM)). Salta Prov., 781m, 244' $38^{\prime \prime} \mathrm{S} 64^{\circ} 01^{\prime} 56^{\prime \prime} \mathrm{W}$, Cabeza de Buey, 21.III.2003, sweeping, J. Munro (1 male, USNMENT00977610 (USNM)). Salta Prov., Hotel Termas grounds, $899 \mathrm{~m}, 25^{\circ} 50^{\prime} 14^{\prime \prime} \mathrm{S} 64^{\circ} 55^{\prime} 55^{\prime \prime} \mathrm{W}$, Rosario de la Frontera, 22.III.2003, sweeping, J. Munro (1 female, USNMENT00993773 (USNM)). Salta Prov., Orán Dept., km33, RN-57, 1.XI-8. XI.1978, Malaise trap, Fidalgo (2 females, USNMENT01197655-01197656 (MLPA)). Salta Prov., Rosario de Lerma, 16.XII-18.XII.1983, Malaise trap, M.
Wasbauer (1 female, USNMENT01081052 (USNM)). Salta Prov., Rosario de Lerma, 17.XI-18.XI.1983, Malaise trap, M. Wasbauer (1 female, USNMENT01081068 (USNM)). Salta Prov., Rosario de Lerma, 21.XII-23.XII.1983, Malaise trap, M. Wasbauer (1 male, USNMENT01081054 (USNM)). Salta Prov., Rosario de Lerma, 24.XII-27. XII.1983, Malaise trap, M. Wasbauer (1 male, USNMENT01081059 (USNM)). Salta Prov., road to San Andrés, along Blanca River, $633 \mathrm{~m}, 23.13^{\circ} \mathrm{S} 63.48^{\circ} \mathrm{W}$, San Ramón de la Nueva Orán, 22. III.2003, sweeping, J. Munro (1 male, USNMENT00977725 (USNM)). Santiago del Estero Prov., 10km ENE Colonia Dora, Salado River, 17.XI-25. XI.1979, C. Vardy \& M. Vardy (9 females, 5 males, USNMENT00970075-00970076, 00970080-00970081, 00970084, 00970086, 00970088, 00970093-00970094, 00970098, 00970104, 00970107, 00970113, 00970125 (BMNH)). Tucumán Prov., Burruyacú, 16.IV-17.VII.1972, Fidalgo (1 female, USNMENT01223586 (MLPA)). Tucumán Prov., herbaceous vegetation, San Javier, 16.II.1981, Fidalgo \& Armesto (5 females, 7 males, USNMENT0119765701197668 (MLPA)). 1999, N. Díaz (1 female, USNMENT00977473 (UCRC)). Córdoba Prov., Córdoba, no date (1 female, USNMENT00977464 (UCRC)). Misiones Prov., XI-2003 - XII-2003, Malaise trap, B. V. Brown \& G. Kung (1 male, USNMENT00977528 (UCRC)). BELIZE: Toledo Dist., Punta Gorda, 12.V-26.V.1982, Kelly \& George (1 female, 2 males, USNMENT00970061, 00970064, 00970068 (BMNH)). Toledo Dist., Punta Gorda, 27.IV-7.V.1982, P. Kelley \& R. S. George (1 male, USNMENT00970051 (BMNH)). BOLIVIA: El Beni Dept., NE of San Borja, El Porvenir Station, 9.VIII.1988, R. W. Brooks (1 female, USNMENT01197714 (SEMC)). BRAZIL: GO, Itumbiara, 15. 
IV.1998, Marchiori (1 male, USNMENT01197679 (MLPA)). GO, Itumbiara, 16.VI.1998, Marchiori (1 male, USNMENT01197677 (MLPA)). GO, Itumbiara, 18.IV.2002, Marchiori (3 females, 2 males, USNMENT0119766901197673 (MLPA)). GO, Itumbiara, 18. VII.2002, Marchiori (1 male, USNMENT01197674 (MLPA)). GO, Itumbiara, 21.IV.1998, Marchiori (1 female, USNMENT01197676 (MLPA)). GO, Itumbiara, 21.VII.1998, Marchiori (1 male, USNMENT01197680 (MLPA)). GO, Itumbiara, 23.VI.1998, Marchiori (1 male, USNMENT01197681 (MLPA)). GO, Itumbiara, 27.VI.1998, Marchiori (1 male, USNMENT01197678 (MLPA)). GO, Itumbiara, 4.VII.1998, Marchiori (1 male, USNMENT01197675 (MLPA)). MG, Araporã, 11.IV.2002, Marchiori (1 female, USNMENT01197697 (MLPA)). MG, Araporã, 16.V.2002, Marchiori (1 female, USNMENT01197698 (MLPA)). MG, Araporã, 18.IV.2002, Marchiori (2 females, 1 male, USNMENT0119768401197685, 01197696 (MLPA)). MG, Araporã, 20.VI.2002, Marchiori (1 male, USNMENT01197695 (MLPA)). MG, Araporã, 23.V.2002, Marchiori (1 male, USNMENT01197692 (MLPA)). MG, Araporã, 25.IV.2002, Marchiori (1 male, USNMENT01197682 (MLPA)). MG, Araporã, 25.VII.2002, Marchiori (1 male, USNMENT01197693 (MLPA)). MG, Araporã, 26.IX.2002, Marchiori (1 female, USNMENT01197683 (MLPA)). MG, Araporã, 29.V.2002, Marchiori (1 male, USNMENT01197694 (MLPA)). MG, Araporã, 5.IX.2002, Marchiori (1 male, USNMENT01197699 (MLPA)). MG, Araporã, 6.VI.2002, Marchiori (1 female, USNMENT01197691 (MLPA)). MG, Araporã, 9.V.2002, Marchiori (3 females, 2 males, USNMENT0119768601197690 (MLPA)). MG, Vicosa, 10. VII.1932, E. J. Hambleton (2 females, USNMENT00977021, 00977562 (USNM)).
PR, Curitiba, III-1954, N. L. H. Krauss (1 male, USNMENT01223599 (USNM)). RJ, Silva Jardim Mpio., Poço das Antas Biological Reserve, 1998, reared, Barbosa da Silva (1 male, USNMENT01197700 (MLPA)). SC, 27¹1'S $52^{\circ} 23^{\prime} \mathrm{W}$, Nova Teutônia, 10.VI.1937, F. Plaumann (1 female, 1 male, USNMENT00969875, 00969887 (BMNH)). SC, $27^{\circ} 11^{\prime} \mathrm{S} 52^{\circ} 23^{\prime} \mathrm{W}$, Nova Teutônia, 10.VII.1937, F. Plaumann (1 male, USNMENT00969854 (BMNH)). SC, $27^{\circ} 11^{\prime} \mathrm{S} 52^{\circ} 23^{\prime} \mathrm{W}$, Nova Teutônia, 13. IV.1938, F. Plaumann (1 male, USNMENT00969885 (BMNH)). SC, 2711'S $52^{\circ} 23^{\prime} \mathrm{W}$, Nova Teutônia, 14.VI.1937, F. Plaumann (1 female, USNMENT00969883 (BMNH)). SC, $27^{\circ} 11^{\prime} \mathrm{S} 52^{\circ} 23^{\prime} \mathrm{W}$, Nova Teutônia, 15.XII.1938, F. Plaumann (5 females, USNMENT00969765-00969766, 00969896, 00969899-00969900 (BMNH)). SC, $27^{\circ} 11^{\prime} \mathrm{S} 52^{\circ} 23^{\prime} \mathrm{W}$, Nova Teutônia, 16.VII.1937, F. Plaumann (1 female, USNMENT00969587 (BMNH)). SC, $27^{\circ} 11^{\prime} \mathrm{S} 52^{\circ} 23^{\prime} \mathrm{W}$, Nova Teutônia, 17. XI.1938, F. Plaumann (1 male, USNMENT00969762 (BMNH)). SC, 2711'S $52^{\circ} 23^{\prime}$ W, Nova Teutônia, 18.XI.1938, F. Plaumann (1 female, USNMENT00969865 (BMNH)). SC, $27^{\circ} 11^{\prime} \mathrm{S} 52^{\circ} 23^{\prime} \mathrm{W}$, Nova Teutônia, 19.V.1937, F. Plaumann (1 female, USNMENT00969772 (BMNH)). SC, $27^{\circ} 11^{\prime} \mathrm{S} 52^{\circ} 23^{\prime} \mathrm{W}$, Nova Teutônia, 19.VIII.1937, F. Plaumann (1 male, USNMENT00969866 (BMNH)). SC, $27^{\circ} 11^{\prime} \mathrm{S} 52^{\circ} 23^{\prime} \mathrm{W}$, Nova Teutônia, 19. XI.1937, F. Plaumann (1 female, USNMENT00969783 (BMNH)). SC, 2711's $52^{\circ} 23^{\prime} \mathrm{W}$, Nova Teutônia, 19.XI.1938, F. Plaumann (1 female, USNMENT00969881 (BMNH)). SC, $27^{\circ} 11^{\prime} \mathrm{S} 52^{\circ} 23^{\prime} \mathrm{W}$, Nova Teutônia, 20.VIII.1935, F. Plaumann (1 male, USNMENT00969816 (BMNH)). SC, $27^{\circ} 11^{\prime} \mathrm{S} 52^{\circ} 23^{\prime} \mathrm{W}$, Nova Teutônia, 24.VI.1937, F. Plaumann (1 female, USNMENT00969874 (BMNH)). SC, $27^{\circ} 11^{\prime} \mathrm{S} 52^{\circ} 23^{\prime} \mathrm{W}$, Nova Teutônia, 25. 
III.1936, F. Plaumann (1 female, USNMENT00969779 (BMNH)). SC, 2711'S $52^{\circ} 23^{\prime} \mathrm{W}$, Nova Teutônia, 25.VI.1937, F. Plaumann (1 female, 2 males, USNMENT00969856, 00969861-00969862 (BMNH)). SC, $27^{\circ} 11^{\prime} \mathrm{S} 52^{\circ} 23^{\prime} \mathrm{W}$, Nova Teutônia, 29.VI.1937, F. Plaumann (4 females, USNMENT00969855, 00969857, 00969870, 00969884 (BMNH)). SC, $27^{\circ} 11^{\prime} \mathrm{S} 52^{\circ} 23^{\prime} \mathrm{W}$, Nova Teutônia, 5. IV.1938, F. Plaumann (1 male, USNMENT00970023 (BMNH)). SC, 27¹1'S $52^{\circ} 23^{\prime} \mathrm{W}$, Nova Teutônia, 6.II.1937, F. Plaumann (1 female, USNMENT00969761 (BMNH)). SC, 27¹1'S $52^{\circ} 23^{\prime} \mathrm{W}$, Nova Teutônia, 6.IV.1938, F. Plaumann (1 female, 2 males, USNMENT00970013, 00970016, 00970018 (BMNH)). SC, $27^{\circ} 11^{\prime} \mathrm{S} 52^{\circ} 23^{\prime} \mathrm{W}$, Nova Teutônia, 7.IV.1938, F. Plaumann (2 females, 2 males, USNMENT0097001900970020, 00970039, 00970073 (BMNH)). $\mathrm{SC}, 27^{\circ} 11^{\prime} \mathrm{S} 52^{\circ} 23^{\prime} \mathrm{W}$, Nova Teutônia, 9. IX.1949, F. Plaumann (1 female, USNMENT00969906 (BMNH)). SC, 2711'S $52^{\circ} 23^{\prime} \mathrm{W}$, Nova Teutônia, II-1937, F. Plaumann (3 females, USNMENT00969758, 00969820, 00969824 (BMNH)). SC, $27^{\circ} 11^{\prime} \mathrm{S} 52^{\circ} 23^{\prime} \mathrm{W}$, Nova Teutônia, XI1935, F. Plaumann (1 male, USNMENT00969589 (BMNH)). SC, $27^{\circ} \mathrm{S}$ $52.58^{\circ} \mathrm{W}$, Nova Teutônia, 2.VI.1938, F. Plaumann (1 female, USNMENT00969567 (BMNH)). SC, $27^{\circ} \mathrm{S} 52.58^{\circ} \mathrm{W}$, Nova Teutônia, 24.III.1938, F. Plaumann (1 male, USNMENT00970009 (BMNH)). SC, $27^{\circ} \mathrm{S} 52.58^{\circ} \mathrm{W}$, Nova Teutônia, 25. III.1938, F. Plaumann (1 female, USNMENT00970031 (BMNH)). SC, $27^{\circ} \mathrm{S}$ $52.58^{\circ} \mathrm{W}$, Nova Teutônia, 3.III.1938, F. Plaumann (3 females, USNMENT00969584-00969585, 00970022 (BMNH)). SC, $27^{\circ} \mathrm{S} 52.58^{\circ} \mathrm{W}$, Nova Teutônia, 3.VI.1938, F. Plaumann (7 females, USNMENT00969559-00969560, 00969563, 00969570-00969572, 00970035 $(\mathrm{BMNH})) . \mathrm{SC}, 27^{\circ} \mathrm{S} 52.58^{\circ} \mathrm{W}$, Nova
Teutônia, 8.VI.1938, F. Plaumann (1 female, USNMENT00969561 (BMNH)). SC, Nova Teutônia, 10.X.1949, F. Plaumann (2 males, USNMENT00969632, 00969635 (BMNH)). SC, Nova Teutônia, 19.V.1937, F. Plaumann (1 female, USNMENT00969895 (BMNH)). SC, Nova Teutônia, II-1937, F. Plaumann (1 female, USNMENT00969842 (BMNH)). SP, Santos, VIII-1961, Krauss (1 female, USNMENT00993938 (USNM)). SP, São Sebastião Mpio., Center for Marine Biology (CEBIMar), São Sebastião, 9. XII.1992, F. Ejchel (1 female, USNMENT01197701 (MZSP)). SP, São Vicente, VIII-1961, N. L. H. Krauss (1 male, USNMENT00977583 (USNM)). COLOMBIA: Cundinamarca Dept., 10mi W Villeta, 15.III.1955, E. I. Schlinger \& E. S. Ross (1 female, USNMENT00977458 (CAS)). Magdalena Dept., Palangana, M.2567, 30m, $11^{\circ} 20^{\prime} \mathrm{N} 74^{\circ} 02^{\prime} \mathrm{W}$, Tayrona Natural National Park, 31.X-15.XI.2001, Malaise trap, R. Henriquez (1 male, USNMENT01223598 (USNM)). Magdalena Dept., Tayrona Natural N.P., M.627, $30 \mathrm{~m}, 11^{\circ} 20^{\prime} \mathrm{N} 74^{\circ} 02^{\prime} \mathrm{W}$, Cañaveral, 30. VIII-19.IX.2000, Malaise trap, R. Henriquez (1 male, USNMENT00977891 (USNM)). Magdalena Dept., Zaino, M.136, 50m, $11^{\circ} 20^{\prime} \mathrm{N} 74^{\circ} 02^{\prime} \mathrm{W}$, Tayrona Natural National Park, 13.V-30. V.2000, R. Henriquez (1 female, USNMENT00977526 (UCRC)). Magdalena Dept., Zaino, M.789, 50m, $11^{\circ} 20^{\prime} \mathrm{N}$ $74^{\circ} 02^{\prime} \mathrm{W}$, Tayrona Natural National Park, 10.X-3.XI.2000, Malaise trap, R. Henriquez (1 female, 1 male, USNMENT00977889-00977890 (USNM)). Magdalena Dept., Zaino, M.793, 50m, $11^{\circ} 20^{\prime} \mathrm{N} 74^{\circ} 02^{\prime} \mathrm{W}$, Tayrona Natural National Park, 29.IX-17.X.2000, Malaise trap, R. Henriquez (10 males, USNMENT00977814-00977822, 00977829 (USNM)). Magdalena Dept., road between Cañaveral and Arrecifes, M.3441, 
$50 \mathrm{~m}, 11^{\circ} 18^{\prime} \mathrm{N} 73^{\circ} 56^{\prime} \mathrm{W}$, Tayrona Natural National Park, 14.I.2003, sweeping, C. Sarmiento (1 male, USNMENT00977888 (USNM)). Norte de Santander Dept., Toledo Mpio., Pozo Negro, Vda. La Camacha, M.4029, 2213m, $7^{\circ} 21^{\prime} \mathrm{N}$ $72^{\circ} 28^{\prime} \mathrm{W}$, Tamá Natural National Park, 23.X-2.XI.2003, Malaise trap, C. Leal (1 female, USNMENT00977887 (USNM)). Valle del Cauca Dept., 6mi W Cali, 1630m, 20.III.1955, E. I. Schlinger \& E. S. Ross (1 female, USNMENT00977457 (CAS)). montane rainforest, $500 \mathrm{~m}$, 054' $\mathrm{N} 76^{\circ} 30^{\prime} \mathrm{W}$, Chocó Dept., 11. IV-13.IV.1973 (4 females, USNMENT00977615-00977617, 00977619 (USNM)). COSTA RICA: Alajuela Prov., NE of Colonia Dos Ríos, La Selva Estate, 400m, 27.III.1988, Hanson (1 female, USNMENT00969984 (BMNH)). Alajuela Prov., Peninsula Path, 600m, $10^{\circ} 27^{\prime} \mathrm{N} 84^{\circ} 40^{\prime} \mathrm{W}$, Arenal Volcano National Park, 25.II.2003, J. Noyes (1 female, 1 male, USNMENT0097797100977972 (USNM)). Cartago Prov., Pejibaye, 24.III-25.III.1987, W. Steiner (1 male, USNMENT01223596 (USNM)). Cartago Prov., Turrialba, 600-700m, 12. VIII.1975, N. L. H. Krauss (1 female, USNMENT00977580 (USNM)). Guanacaste Prov., $13 \mathrm{~km}$ E Filadelfia, 40m, V-1989 - VI-1989, Malaise trap, M. Garcia (1 male, USNMENT00993824 (USNM)). Guanacaste Prov., 20km SW Cañas, Enrique Jimenez Nuñez Experimental Station, 5.XI-17.XI.1991, Malaise trap, A. S. Menke (1 male, USNMENT00977857 (USNM)). Guanacaste Prov., H.3.O, Santa Rosa National Park, 300m, 14.VIII-6.IX.1985, Malaise trap, D. Janzen \& I. Gauld (1 female, USNMENT01223601 (USNM)). Limón Prov., Cahuita, 23.XII-25.XII.1988, Malaise trap, A. S. Menke (1 female, USNMENT00993955 (USNM)). Puntarenas Prov., Osa Peninsula, house of C. L. Madrigal, Puerto Jiménez, 10m, I-1991,
Malaise trap (3 females, 2 males, USNMENT00977756-00977757, 00977836, 00977840, 00977842 (USNM)). Puntarenas Prov., Osa Peninsula, house of C. L. Madrigal, Puerto Jiménez, 10m, I-1991, Malaise trap, P. Hanson (1 male, USNMENT01223600 (USNM)). Puntarenas Prov., Osa Peninsula, house of C. L. Madrigal, Puerto Jiménez, 10m, II-1991, Malaise trap (1 female, USNMENT00977752 (USNM)). Puntarenas Prov., Osa Peninsula, house of C. L. Madrigal, Puerto Jiménez, 10m, III-1992, Malaise trap (1 female, USNMENT00977760 (USNM)). Puntarenas Prov., Osa Peninsula, house of C. L. Madrigal, Puerto Jiménez, 10m, VIII-1991, Malaise trap (1 female, 1 male, USNMENT00977761-00977762 (USNM)). Puntarenas Prov., Puerto Jiménez, III-1991 (1 female, USNMENT00977738 (USNM)). Puntarenas Prov., Puerto Jiménez, IV-1991 (1 female, USNMENT00977745 (USNM)). Puntarenas Prov., Puerto Jiménez, VII1991 (1 female, USNMENT00977742 (USNM)). San José Prov., Escazú Canton, San Antonio de Escazú, 1300m, V-1987, Eberhard (1 male, USNMENT00969911 (BMNH)). San José Prov., Sector Sur, Hatillo 5, 1100m, 19. III.1997, K. Nishida (1 female, USNMENT00977928 (USNM)). DOMINICA: Saint George Parish, Roseau, 0-100m, VII-1976, N. L. H. Krauss (1 female, USNMENT00993987 (USNM)). Saint Paul Parish, Springfield, 2002/006 +007 , 965ft, $\quad 15^{\circ} 20.749^{\prime} \mathrm{N} \quad 61^{\circ} 22.147^{\prime} \mathrm{W}$, Archbold Tropical Research Center (ATRC), 31.V-14.VI.2002, Malaise trap, Marable, Wolman et al. (1 female, USNMENT00977667 (USNM)). Saint Paul Parish, site 2, Mount Joy, 5.VI-8. VI.1991, Malaise trap, H. Geitzenauer (1 female, USNMENT00977402 (TAMU)). DOMINICAN REPUBLIC: Puerto Plata Prov., among reeds / wetlands, $16 \mathrm{~m}$, $19.76049^{\circ} \mathrm{N} \quad 70.42979^{\circ} \mathrm{W}, \quad$ Cabarete, 
12-IX-2008, sweeping, H. Clebsch (1 female, USNMENT00977975 (USNM)). ECUADOR: Carchi Prov., Ibarra-Tulcan section of Pan American Highway, km35, Chota River, 2.I.1990, Malaise trap, M. Wasbauer \& J. Wasbauer (1 male, USNMENT01081001 (USNM)). Los Ríos Prov., Palenque River, 5. II.1983, L. Huggert (1 unknown, USNMENT00977602 (USNM)). Napo Prov., Puerto Misahuallí, 20.II.1983, L. Huggert (1 female, USNMENT00977395 (TAMU)). Santo Domingo de los Tsáchilas Prov., Tinalandia, 2.II.1983, L. Huggert (3 females, USNMENT00977392-00977394 (TAMU)). Santo Domingo de los Tsáchilas Prov., Tinalandia, 800m, 2.II.1983, Masner \& Sharkey (4 females, 10 males, USNMENT00977587-00977600 (USNM)). EL SALVADOR: La Libertad Prov., Nueva San Salvador (Santa Tecla), 900950m, VIII-1975, N. L. H. Krauss (1 female, USNMENT00977581 (USNM)). San Salvador Dept., San Salvador, 4. V.1958, O. L. Cartwright (1 male, USNMENT00977560 (USNM)). GUADELOUPE: Basse-Terre Arrond., Capesterre-Belle-Eau, GP 2207, Grand Étang, 18.VI.2004, reared, J. Etienne (4 females, USNMENT00977931-00977934 (USNM)). Basse-Terre Arrond., at feet of Morne Houë1, GP 2193, Saint-Claude, 13.V.2004, reared, J. Etienne (1 female, USNMENT00977951 (USNM)). GP 1060, Grand Étang, 7.II.1995, reared, J. Etienne (3 females, USNMENT00977927, 00977930, 00977935 (USNM)). GUATEMALA: Escuintla Dept., Escuintla, 20.VIII.1975, N. L. H. Krauss (1 female, USNMENT00977584 (USNM)). GUYANA: Cuyuni-Mazaruni Reg., clearing / under tree, Mazaruni, 26.VIII.1937, Richards \& Smart (1 male, USNMENT00969590 (BMNH)). HONDURAS: Francisco Morazán Dept., Tegucigalpa, 1000-1200m, VIII-1975, N. L. H. Krauss (1 male, USNMENT00977586 (USNM)). MEXICO:
CHIS, Palenque, 23.VII.1984, G. Gordh (1 female, 1 male, USNMENT00977435, 00977437 (UCRC)). CHIS, primary forest, $6.2 \mathrm{mi} \mathrm{N}$ Berriozabal, 4000ft, 8.VIII-9.VIII.1990, J. B. Woolley (1) female, USNMENT00977391 (TAMU)). TAMPS, 6.5mi NW Ciudad Victoria, La Libertad Canyon, 21.III.1986, G. Zolnerowich \& R. Trevino (1 female, USNMENT00977382 (TAMU)). TAMPS, $8.2 \mathrm{~km}$ E Ciudad Victoria, MEX-70, 3. VII.1986, G. Zolnerowich \& R. Trevino (1 female, 1 male, USNMENT0097738700977388 (TAMU)). TAMPS, 97km E Ciudad Victoria, MEX-70, 3.VII.1986, Zolnerowich \& Trevino (1 male, USNMENT00977405 (TAMU)). TAMPS, 97km E Ciudad Victoria, MEX-70, 3. VII.1986, G. Zolnerowich \& R. Trevino (1 female, 2 males, USNMENT00977372, 00977378, 00977380 (TAMU)). TAMPS, Los Cedros Field Station, 340m, $23^{\circ} 03^{\prime} 00^{\prime \prime} \mathrm{N} 99^{\circ} 09^{\prime} 03^{\prime \prime} \mathrm{W}$, Gómez Farías, 14.IV.2002, Malaise trap, A. CordobaTorres (1 male, USNMENT00977812 (USNM)). TAMPS, nr. Llera de Canales, shore of Guayalejo River, 7.III.2002, V. V. Berezovskiy, S. N. Myartseva \& D. R. Kasparian (1 female, USNMENT00977440 (UCRC)). VER, 97/ 015, 0.7mi N Jilotepec, 3680ft, 14. VI.1997, screen sweeping, L. A. Wilson \& J. B. Woolley (1 male, USNMENT00977416 (TAMU)). VER, Huatusco, 8.I-12.I.2007, yellow pan trap, S. Droege (1 female, USNMENT00993843 (USNM)). VER, Ixtaczoquitlán Mpio., $3 \mathrm{~km}$ SW Fortín de las Flores, 97/035, Metlac Park, 2850ft, 23.VI.1997, screen sweeping, J. B. Woolley (1 female, USNMENT00977418 (TAMU)). VER, by Actopan River, 3mi N José Cardel, 31.X.1982, screen sweeping, J. T. Huber \& A. Gonzalez (1 male, USNMENT00977441 (UCRC)). VER, nr. Puente Nacional, 097/013, El Crucero, 13.VI.1997, L. A. Wilson \& J. B. 
Woolley (1 male, USNMENT00977397 (TAMU)). NICARAGUA: León Dept., Finca Manuel Ignacio Lacayo (MIL), XII-1989, J. Tellez (1 female, 1 male, USNMENT01197702-01197703 (MLPA)). Matagalpa, $1300 \mathrm{~m}, 12^{\circ} 59^{\prime} 51^{\prime \prime} \mathrm{N} 85^{\circ} 55^{\prime} 47^{\prime \prime} \mathrm{W}$, Santo Maria de Ostuma, XI-1959, N. L. H. Krauss (1 female, USNMENT00993990 (USNM)). PANAMA: Chiriquí Prov., 2001/026, 630m, $08^{\circ} 40^{\prime} 04^{\prime \prime} \mathrm{N} \quad 82^{\circ} 14^{\prime} 41^{\prime \prime} \mathrm{W}, \quad 6 \mathrm{~km} \quad \mathrm{NW}$ Hornito, 7.I.2001, screen sweeping, M. Yoder \& J. B. Woolley (1 female, USNMENT00993837 (USNM)). Panama, Canal Zone, Paraiso, 17.I.1911, A. Busck (1 female, USNMENT00977559 (USNM)). PARAGUAY: San Pedro Dept., Ypané River, Cororó, 1.XII-4. XII.1983, Malaise trap, M. Wasbauer (1 male, USNMENT01081050 (USNM)). San Pedro Dept., Ypané River, Cororó, 5.XII-9.XII.1983, Malaise trap, M. Wasbauer (2 males, USNMENT01081064, 01081067 (USNM)). PERU: Junín Reg., Perené River, 25.III.1910, C. H. T. Townsend (1 female, USNMENT00977853 (USNM)). Junín Reg., Perené River, 29. III.1910, C. H. T. Townsend (1 female, USNMENT00977906 (USNM)). Lima Reg., Chancay River Valley, 15.III.1951, Michelbacher \& Ross (1 female, USNMENT00977455 (CAS)). Loreto Reg., 80km NE Iquitos, Amazon River, Explorama Lodge, 24.VI-20.VII.1990, Malaise trap, Menke \& Avertschenko (1 female, USNMENT00977855 (USNM)). PUERTO RICO: Jayuya Mpio., Cerro de Punta, VII-1962, J. Maldonado C. (1 female, USNMENT00977571 (USNM)). SAINT VINCENT AND THE GRENADINES: Saint Andrew Parish, rainforest edge, Maloney, 12.VII.1976, J. S. Noyes (1 female, USNMENT00969548 $(\mathrm{BMNH})$ ). Saint David Parish, banana plantation, Richmond, 10.VII.1976, J. S. Noyes (1 female, USNMENT00969552 $(\mathrm{BMNH})$ ). wasteground, Wallilabou, 12.
VII.1976, J. S. Noyes (1 female, USNMENT00969545 (BMNH)). TRINIDAD AND TOBAGO: Mayaro/Rio Claro Reg., Trinidad Isl., cocoa plantation, Ecclesville, 28.VII.1976, J. S. Noyes (1 female, USNMENT00969676 (BMNH)). Tobago Isl., Crown Point, Friendship, 28. X.1977, S. L. Ward (1 female, USNMENT00970054 (BMNH)). Tobago Isl., rainforest edge, Parlatuvier Valley, 20. VII.1976, J. S. Noyes (1 female, 2 males, USNMENT00969535-00969537 (BMNH)). Trinidad Isl., Maracas Valley, XII-1977, Malaise trap, F. D. Bennett (1 female, USNMENT00969610 (BMNH)). Tunapuna/Piarco Reg., Trinidad Isl., Saint Augustine, 26.VII.1949, E. McCallan (2 males, USNMENT00977561, 00977565 (USNM)). Tunapuna/Piarco Reg., Trinidad Isl., Saint Augustine, II-1954 (1 unknown, USNMENT00977461 (CAS)). Tunapuna/Piarco Reg., Trinidad Isl., Saint Augustine, IX-1976, Malaise trap, F. D. Bennett (1 female, USNMENT00969708 (BMNH)). Tunapuna/ Piarco Reg., Trinidad Isl., Saint Augustine, XI-1976, Malaise trap, F. D. Bennett (1 female, USNMENT00969605 (BMNH)). Tunapuna/Piarco Reg., Trinidad Isl., wasteground, Saint Augustine, 24. VI.1976, J. S. Noyes (1 female, USNMENT00969795 (BMNH)). Tunapuna/ Piarco Reg., Trinidad Isl., wasteground, Saint Augustine, 25.VI.1976, J. S. Noyes (1 female, USNMENT00969677 (BMNH)). Tunapuna/Piarco Reg., Trinidad Isl., wasteground, Saint Augustine, no date, J. S. Noyes (1 male, USNMENT00969555 (BMNH)).

Distribution.-Neotropical Region: Cuba, Panama, Brazil and Argentina (Kieffer 1907; Díaz and Gallardo 1997; Marchiori and Oliveira 2001; Fontal and Nieves-Aldrey 2004). Belize, Bolivia, Colombia, Costa Rica, Dominica, Dominican Republic, Ecuador, El Salvador, Guadeloupe, Guatemala, Guyana, Honduras, 
Mexico, Nicaragua, Peru, Puerto Rico, Saint vincent and the Grenadines and Trinidad and Tobago (new records) and new provinces record for Argentina: Buenos Aires, Cordoba, Salta and Tucuman and new states records for Brazil: Minas Gerais, Rio de Janeiro and Sao Paulo.

Biology.-Recorded hosts are Diptera: Agromyzidae: Nemorimyza maculosa (Malloch) (in Buffington 2009); Calycomyza servilis and Liryomyza huidobrensis are new records reported here (based on label data).

\section{Zaeucoila infuscata Gallardo and Buffington, new species}

urn:1sid:zoobank.org:act:102E27EC-41C4460D-B9F8-BDFA14CEA70E

http://bioguid.osu.edu/xbiod_concepts/ 408247

(Figs. 38-43)

Description.-Coloration with head, mesosoma, metasoma black to dark brown; legs with coxae and hind femora dark brown, rest yellow. Lateral margin of occiput variable, ranging from gently scalloped to smooth, sharp carina. Vertical carina adjacent to ventral margin of antennal socket present, leading to deep inner orbital groove. Malar space adjacent to anterior articulation of mandible with short, blunt, smooth protuberance. Malar sulcus present, composed of series of small striae dorsally, leading to single line ventrally. Orbital furrows sharp, divergent groove running from antennal sockets to dorsal end of malar sulcus. Dorsal aspect of vertex smooth with a few short, scattered setae. Posterior aspect of vertex smooth. Hair punctures on lateral aspect of vertex absent. Placoidal sensilla present on $\mathrm{F} 2-11$. Pubescence on lateral surface of pronotum present, sparse, with distinct row behind pronotal plate. Ridges extending posteriorly from lateral margin of pronotal plate present, typically short, with single prominent ridge extending to anterior margin of mesoscutum. Sculpture on mesoscutum absent, with sparse long hairs. Median mesoscutal carina present, composed of anteriorly broad elevation, narrowing posteriorly, ending at midline of mesoscutum. Anterior admedial lines present, in conjunction with mesoscutal carina. Lateroventral mesopleural carina present, marking abrupt change of slope of mesopectus, terminating anteriorly at large, oblique notch. Mesopleural triangle present, gently impressed with distinct dorsal border, ventral border lacking; smooth. Dorsal surface of scutellum laterally with distinct ridges radiating from plate, posteriorly with deep fovea and ridges. Latero-ventral margin of scutellum posterior to auricula smooth, with weak longitudinal striae. Subalar area abruptly broadened anteriorly, with an indicated longitudinal division. Pubescence present, but very sparse, along posterior and ventral margins of metapleuron; short and dense on propodeum. Pubescence of fore wing mesally sparse, gradually becoming denser distally. Coloration of wing variable, ranging entirely infuscate basally to blotchy infuscation basally. Hair fringe along apical margin of fore wing present, with particularly longer setae along the mesal anterior margin. Inter propodeal carinae space moderately setose, with long, thin setae evenly filling the space; surface underneath smooth.

Diagnosis. - This species is unique in its possession of a heavily infuscate mesal $1 / 3$ of the forewing. This character is also shared with $Z$. grenadensis, but can be separated from this species by the lack of ocellar hair patches and parapsidal hair lines (both present in $Z$. grenadensis). 

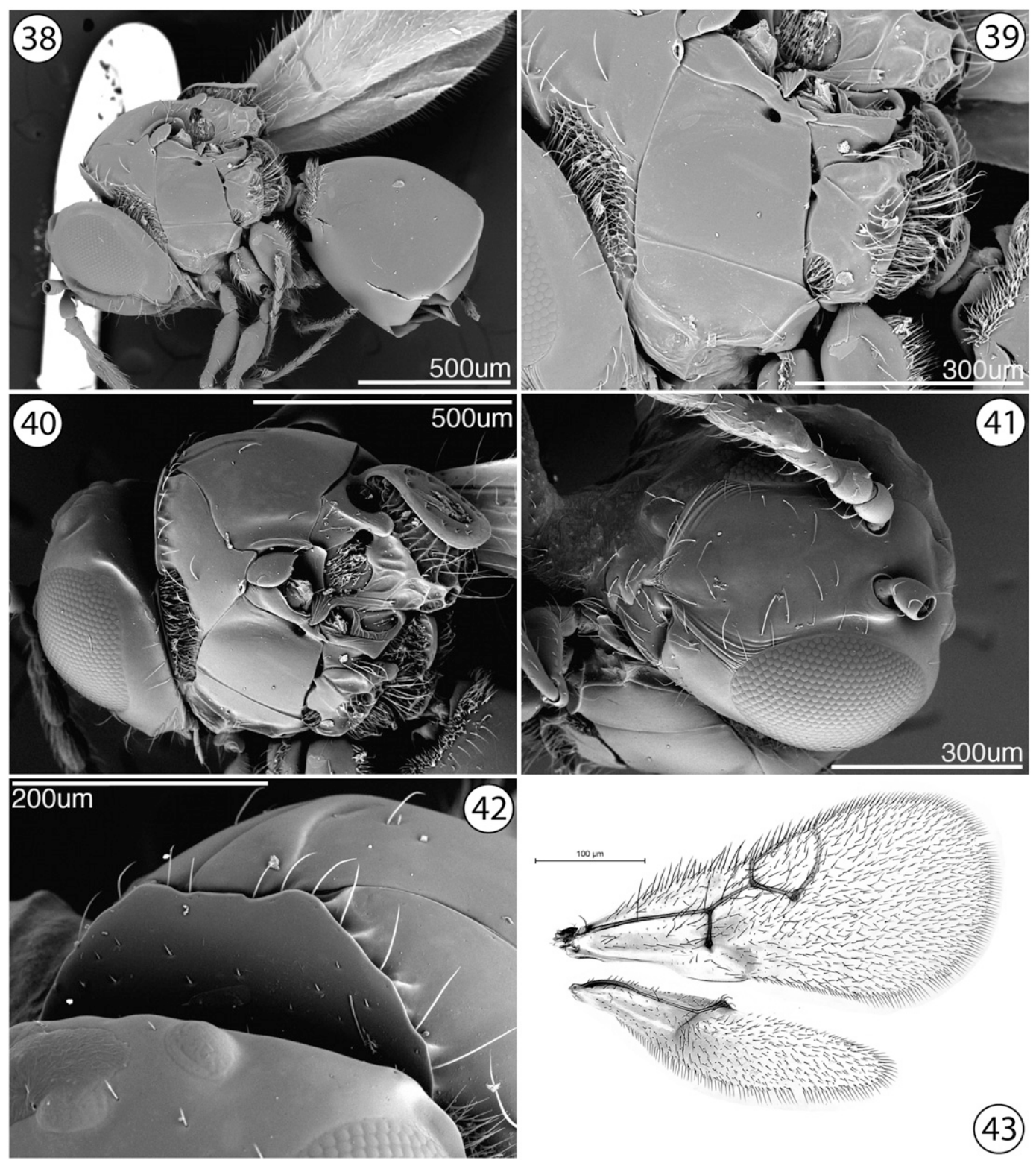

Plate 9, Figs. 38-43. Zaeucoila infuscata, new species. 38, habitus, lateral view; 39, mesopleuron and metapleuron, lateral view; 40, head and mesosoma, postero-dorsal view; 41, head, anterior view; 42, pronotal plate, antero-dorsal view; 43, fore and hind wings.

Etymology.- Name refers to the infuscation at the base of the fore and hind wings.

Link to Distribution Map.-[http:// hol.osu.edu/map-full.html?id=408247]

Material Examined.-Holotype, male: ECUADOR: Napo Prov., km 45 on Hollín-Loreto Road, Huahua Sumaco, 15.XII.1989, Malaise trap, M. Wasbauer, J. Wasbauer \& H. Real, USNMENT01081072 (deposited in USNM). Paratypes: (2 females, 3 males) ECUADOR: Napo Prov., km 44 on 
Hollín-Loreto Road, Huahua Sumaco, 16.XII.1989, Malaise trap, M. Wasbauer, J. Wasbauer \& H. Real (1 female, 1 male, USNMENT01081070, 01081074 (USNM)). Napo Prov., km 45 on HollínLoreto Road, Huahua Sumaco, 15. XII.1989, Malaise trap, M. Wasbauer \& J. Wasbauer (1 female, USNMENT01081071 (USNM)). Napo Prov., km 45 on Hollín-Loreto Road, Huahua Sumaco, 21.XII.1989, Malaise trap, M. S. Wasbauer, J. S. Wasbauer \& H. Real (1 male, USNMENT01081069 (USNM)). Napo Prov., km 45 on Hollín-Loreto Road, Huahua Sumaco, 21.XII.1989, Malaise trap, M. Wasbauer, J. Wasbauer \& H. Real (1 male, USNMENT01081073 (USNM)). Other material: (82 females, 77 males) ARGENTINA: Misiones Prov., Iguazú National Park, 140m, 10.IV-11.IV.1974, Malaise trap, C. R. Vardy (1 female, USNMENT00969639 (BMNH)). Misiones Prov., Iguazú National Park, 140m, 8. IV-11.IV.1974, Malaise trap, C. Vardy \& M. Vardy (1 male, USNMENT00969645 $(\mathrm{BMNH}))$. Misiones Prov., Posadas, V-1961, N. L. H. Krauss (1 female, USNMENT00977585 (USNM)). Misiones Prov., San Ignacio, V-1961, Krauss (1 female, USNMENT00993930 (USNM)). Misiones Prov., nr. Loreto, wet forest, $77 \mathrm{~m}, 27.34^{\circ} \mathrm{S} 55.53^{\circ} \mathrm{W}$, Santa Ana, 27. III.2003, sweeping, J. Munro (1 female, USNMENT00977611 (USNM)). Salta Prov., 66km E jct. RN-34, dry chaco,

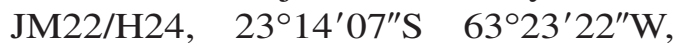
RN 81, 24.III.2003, sweeping, J. Munro (1 female, USNMENT00977608 (USNM)). Salta Prov., Rosario de Lerma, 4.XI-8. XI.1983, Malaise trap, M. Wasbauer (1 male, USNMENT01081049 (USNM)).

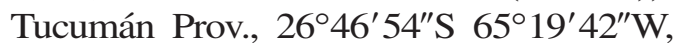
nr. San Miguel de Tucumán, 20.I.2003, S. Triapitsyn (1 male, USNMENT00977430 (UCRC)). BOLIVIA: El Beni Dept., 40m E San Borja, Palm Camp at Rio Curiraba, riverbank sand bar, Beni
Biological Station, 9.IX-15.IX.1987, Malaise trap, W. E. Steiner (3 males, USNMENT00977860-00977862 (USNM)). Santa Cruz Dept., $18^{\circ} 06^{\prime} \mathrm{S} 63^{\circ} 36^{\prime} \mathrm{W}, \mathrm{El}$ Refugio Los Volcanes, 25.X-30.X.2007, Malaise trap, J. Romero \& F. Romero (1 male, USNMENT00977968 (USNM)). BRAZIL: GO, Campinaçu, 19.II-23.II.1996, Malaise trap (1 male, USNMENT01223495 (MZSP)). GO, Itumbiara, 11.IV.2002, Marchiori (1 male, USNMENT01223493 (MLPA)). GO, Itumbiara, 18.IV.2002, Marchiori (1 male, USNMENT01223494 (MLPA)). MG, Araporã, 10.X.2002, Marchiori (1 male, USNMENT01223492 (MLPA)). MG, Araporã, 13.VI.2002, Marchiori (1 male, USNMENT01197709 (MLPA)). MG, Araporã, 16.V.2002, Marchiori (2 males, USNMENT0119770401197705 (MLPA)). MG, Araporã, 25. VII.2002, Marchiori (1 male, USNMENT01197710 (MLPA)). MG, Araporã, 26.IX.2002, Marchiori (2 males, USNMENT01223490-01223491 (MLPA)). MG, Araporã, 29.V.2002, Marchiori (1 male, USNMENT01197706 (MLPA)). MG, Araporã, 5.IX.2002, Marchiori (2 males, USNMENT01197711-01197712 (MLPA)). MG, Araporã, 6.VI.2002, Marchiori (2 males, USNMENT01197707-01197708 (MLPA)). SC, $27^{\circ} 11^{\prime} \mathrm{S} 52^{\circ} 23^{\prime} \mathrm{W}$, Nova Teutônia, 10.IX.1938, F. Plaumann (1 male, USNMENT00969785 (BMNH)). SC, $27^{\circ} 11^{\prime} \mathrm{S} 52^{\circ} 23^{\prime} \mathrm{W}$, Nova Teutônia, 10.VII.1937, F. Plaumann (1 male, USNMENT00969858 (BMNH)). SC, $27^{\circ} 11^{\prime} \mathrm{S} 52^{\circ} 23^{\prime} \mathrm{W}$, Nova Teutônia, 12. VII.1935, F. Plaumann (1 male, USNMENT00969829 (BMNH)). SC, 27¹1'S $52^{\circ} 23^{\prime} \mathrm{W}$, Nova Teutônia, 15.XII.1938, F. Plaumann (1 female, 3 males, USNMENT00969778, 00969859-00969860, 00969880 (BMNH)). SC, $27^{\circ} 11^{\prime} \mathrm{S}$ $52^{\circ} 23^{\prime} \mathrm{W}$, Nova Teutônia, 17.XI.1938, F. Plaumann (1 female, USNMENT00969763 (BMNH)). SC, $27^{\circ} 11^{\prime} \mathrm{S} \quad 52^{\circ} 23^{\prime} \mathrm{W}$, Nova Teutônia, 18.V.1937, F. Plaumann 
(1 female, USNMENTO0969771 (BMNH)). SC, $27^{\circ} 11^{\prime} \mathrm{S} 52^{\circ} 23^{\prime} \mathrm{W}$, Nova Teutônia, 18.XI.1938, F. Plaumann (1 male, USNMENT00969863 (BMNH)). SC, $27^{\circ} 11^{\prime} \mathrm{S} 52^{\circ} 23^{\prime} \mathrm{W}$, Nova Teutônia, 19. V.1937, F. Plaumann (1 female, USNMENT00969770 (BMNH)). SC, 2711'S $52^{\circ} 23^{\prime} \mathrm{W}$, Nova Teutônia, 19.XI.1937, F. Plaumann ( 2 females, USNMENT00969760, 00969789 (BMNH)). SC, $27^{\circ} 11^{\prime} \mathrm{S}$ $52^{\circ} 23^{\prime} \mathrm{W}$, Nova Teutônia, 22.VIII.1935, F. Plaumann (1 male, USNMENT00969832 (BMNH)). SC, $27^{\circ} 11^{\prime} \mathrm{S} 52^{\circ} 23^{\prime} \mathrm{W}$, Nova Teutônia, 27.IV.1938, F. Plaumann (2 females, USNMENT00969776-00969777 (BMNH)). SC, $27^{\circ} 11^{\prime} \mathrm{S} 52^{\circ} 23^{\prime} \mathrm{W}$, Nova Teutônia, 3.V.1938, F. Plaumann (1 male, USNMENT00969782 (BMNH)). SC, $27^{\circ} 11^{\prime} \mathrm{S} 52^{\circ} 23^{\prime} \mathrm{W}$, Nova Teutônia, 5. IV.1938, F. Plaumann (1 female, USNMENT00970025 (BMNH)). SC, 2711'S $52^{\circ} 23^{\prime} \mathrm{W}$, Nova Teutônia, 6.IV.1938, F. Plaumann ( 2 females, USNMENT00970015, 00970021 (BMNH)). SC, $27^{\circ} 11^{\prime} \mathrm{S} 52^{\circ} 23^{\prime} \mathrm{W}$, Nova Teutônia, 6.V.1938, Plaumann (1 female, USNMENT00969791 (BMNH)). SC, $27^{\circ} 11^{\prime} \mathrm{S} 52^{\circ} 23^{\prime} \mathrm{W}$, Nova Teutônia, 7. IV.1938, F. Plaumann (1 male, USNMENT00970074 (BMNH)). SC, 2711'S $52^{\circ} 23^{\prime}$ W, Nova Teutônia, 9.VIII.1935, F. Plaumann (1 male, USNMENT00969831 (BMNH)). SC, $27^{\circ} 11^{\prime} \mathrm{S} 52^{\circ} 23^{\prime} \mathrm{W}$, Nova Teutônia, II-1937, F. Plaumann (1 female, USNMENT00969775 (BMNH)). SC, $27^{\circ} 11^{\prime} \mathrm{S} 52^{\circ} 23^{\prime} \mathrm{W}$, Nova Teutônia, VIII-1935, F. Plaumann (2 males, USNMENT00969818, 00969828 (BMNH)). SC, $27^{\circ} \mathrm{S} 52.58^{\circ} \mathrm{W}$, Nova Teutônia, 2. VI.1938, F. Plaumann (1 female, USNMENT00969565 (BMNH)). SC, $27^{\circ} \mathrm{S}$ $52.58^{\circ} \mathrm{W}$, Nova Teutônia, 3.VI.1938, F. Plaumann (1 female, USNMENT00970034 $(\mathrm{BMNH})) . \quad \mathrm{SC}, 27^{\circ} \mathrm{S} 52.58^{\circ} \mathrm{W}$, Nova Teutônia, 7.IV.1938, F. Plaumann (1 male, USNMENT00970026 (BMNH)). COLOMBIA: Cundinamarca Dept., Anolaima, III-1993, I. Zenner (1 female,
1 male, USNMENT00977567-00977568 (USNM)). Magdalena Dept., Zaino, M.136, 50m, $11^{\circ} 20^{\prime} \mathrm{N} 74^{\circ} 02^{\prime} \mathrm{W}$, Tayrona Natural National Park, 13.V-30.V.2000, Malaise trap, R. Henriquez (1 male, USNMENT00977491 (UCRC)). Valle del Cauca Dept., by sugarcane field, $10 \mathrm{~km}$ S Cali, 1000m, 3.IV.1971, W. Eberhard \& C. Garcia (2 females, USNMENT00977401 (TAMU); USNMENT00977626 (USNM)). Valle del Cauca Dept., by sugarcane field, $10 \mathrm{~km} \mathrm{~S}$ Cali, 1000m, 3.IV.1991, W. Eberhard \& C. Garcia (15 females, USNMENT0097762100977622, 00977625, 00977670-00977674, 00977676-00977678, 00977681-00977684 (USNM)). montane rainforest, $500 \mathrm{~m}$, $05^{\circ} 45^{\prime} \mathrm{N} 76^{\circ} 30^{\prime} \mathrm{W}$, Chocó Dept., 11.IV-13. IV.1973 (2 females, USNMENT0097762700977628 (USNM)). COSTA RICA: Alajuela Prov., Peninsula Path, 600m, $10^{\circ} 27^{\prime} \mathrm{N} 84^{\circ} 40^{\prime} \mathrm{W}$, Arenal Volcano National Park, 25.II.2003, J. Noyes (1 female, USNMENT00977970 (USNM)). Cartago Prov., NE of Jabillos, Rio Chitaria, 750m, 28.IV.1988, Hanson (1 female, USNMENT00969960 (BMNH)). Puntarenas Prov., Osa Peninsula, house of C. L. Madrigal, Puerto Jiménez, 10m, II-1991, Malaise trap (1 male, USNMENT00977754 (USNM)). Puntarenas Prov., Osa Peninsula, house of C. L. Madrigal, Puerto Jiménez, 10m, II-1991, Malaise trap, P. Hanson (1 male, USNMENT01223592 (USNM)). Puntarenas Prov., Puerto Jiménez, I-1991 (1 female, 5 males, USNMENTO0977726-00977728, 00977730, 00977732, 00977734 (USNM)). Puntarenas Prov., Puerto Jiménez, II1991 (2 males, USNMENT0097773500977736 (USNM)). Puntarenas Prov., Puerto Jiménez, II-1991 - VII-1991 (1 female, USNMENT00977747 (USNM)). Puntarenas Prov., Puerto Jiménez, II-1992 (1 male, USNMENT00977739 (USNM)). Puntarenas Prov., Puerto Jiménez, VI1992 (1 female, USNMENT00977746 
(USNM)). Puntarenas Prov., Puerto Jiménez, XI-1991 (1 female, USNMENT00977743 (USNM)). Puntarenas Prov., Puerto Jiménez, XII-1990 (1 male, USNMENT00977741 (USNM)). Puntarenas Prov., San Vito, Wilson (Las Cruces) Botanical Garden, 1200m, 5. VI.1988, P. Hanson (1 female, 1 male, USNMENT00969947, 00969958 (BMNH)). San José Prov., San Isidro (San Isidro del General), 22.VII.1981, N. L. H. Krauss (1 female, USNMENT00977446 (UCRC)). DOMINICA: Saint Paul Parish, Springfield, 94/017, Archbold Tropical Research Center (ATRC), 27.V-12.VI.1994, Malaise trap, J. B. Woolley (1 female, USNMENT00977403 (TAMU)). ECUADOR: Napo Prov., 10km NE Tena, 400m, 19. II-20.II.1983, L. Masner (4 females, 8 males, USNMENT00977688, 0097769000977692, 00977694-00977698, 0097770200977704 (USNM)). Napo Prov., Puerto Misahuallí, 18.II.1983, L. Huggert (1 female, USNMENT00977604 (USNM)). Napo Prov., Puerto Misahuallí, 20.II.1983, L. Huggert (1 male, USNMENT00977396 (TAMU)). Santo Domingo de los Tsáchilas Prov., Tinalandia, 2.II.1983, Masner \& Sharkey (1 female, USNMENT00977601 (USNM)). GUADELOUPE: Basse-Terre Arrond., Capesterre-Belle-Eau, GP 2312, Grand Étang, 16.IX.2005, reared, J. Etienne (3 females, USNMENT0097777500977776, 00977778 (USNM)). MEXICO: TAMPS, 97km E Ciudad Victoria, MEX70, 3.VII.1986, Zolnerowich \& Trevino (1 female, USNMENT00977404 (TAMU)). VER, 20 $11^{\prime} 20^{\prime \prime} \mathrm{N} 96^{\circ} 51^{\prime} 57^{\prime \prime} \mathrm{W}$, San Raphael Jicaltepec, no date (1 female, USNMENT00977563 (USNM)). NICARAGUA: León Dept., Izapa, 5. VI.1994 (1 male, USNMENT01223496 (MLPA)). PANAMA: Colón Prov., 99/ 033, 120m, $09^{\circ} 19^{\prime} 19^{\prime \prime} \mathrm{N} 79^{\circ} 47^{\prime} 54^{\prime \prime} \mathrm{W}$, $2 \mathrm{~km}$ S Sabanitas, 4.VII-15.VII.1999, Malaise trap, Gillogly \& Woolley (1 male, USNMENT00977398 (TAMU)).
Veraguas Prov., 8km W Santa Fe, 99/ 078, 3000ft, $08^{\circ} 30^{\prime} 26^{\prime \prime} \mathrm{N} 81^{\circ} 06^{\prime} 49^{\prime \prime} \mathrm{W}$, Tute Hill, 24.VII.1999, J. B. Woolley (1 female, USNMENT00977400 (TAMU)). PARAGUAY: Asunción Dist. Capital, Asunción, VII-1961, N. L. H. Krauss (1 female, 1 male, USNMENT0099392800993929 (USNM)). San Pedro Dept., Ypané River, Cororó, 1.XII-4.XII.1983, Malaise trap, M. Wasbauer (1 female, USNMENT01081065 (USNM)). San Pedro Dept., Ypané River, Cororó, 5. XII-9.XII.1983, Malaise trap, M. Wasbauer (5 females, 6 males, USNMENT0108104701081048, 01081053, 01081055-01081058, 01081060-01081063 (USNM)). PERU: Junín Reg., Perené River, 27.III.1910, C. H. T. Townsend (3 females, 1 male, USNMENT00977570, 00977854, 0099394200993943 (USNM)). Lima Prov., Magdalena, 28.III.1910, C. H. T. Townsend (2 females, USNMENTO0993940-00993941 (USNM)). Loreto Reg., 80km NE Iquitos, Amazon River, Explorama Lodge, 24. VI-20.VII.1990, Malaise trap, Menke \& Avertschenko (1 female, USNMENT00977856 (USNM)). Loreto Reg., Maynas Prov., 80km NE Iquitos, Amazon River, Explorama Lodge, 24.VI-20. VII.1990, Malaise trap, Menke \& Avertschenko (2 females, USNMENT00977868-00977869 (USNM)). Ucayali Reg., Yurac, 67mi E Tingo María, 16.XI.1954, E. I. Schlinger \& E. S. Ross (1 male, USNMENT00977451 (CAS)). SAINT VINCENT AND THE GRENADINES: wasteground, Wallilabou, 12.VII.1976, J. S. Noyes (1 female, USNMENT00969556 (BMNH)). TRINIDAD AND TOBAGO: Diego Martin Reg., Trinidad Isl., Point Gourde, 8.VIII.1976, J. S. Noyes (1 female, USNMENT00969664 (BMNH)). Tobago Isl., Courland River, Turtle Beach, 19. X.1977, S. L. Ward (1 male, USNMENT00970053 (BMNH)). Trinidad Isl., Maracas Valley, X-1977, Malaise 
trap, F. D. Bennett (1 female, USNMENT00969615 (BMNH)). Tunapuna/ Piarco Reg., Trinidad Isl., Saint Augustine, IX-1976, Malaise trap, F. D. Bennett (1 female, 1 male, USNMENT00969705, 00969744 (BMNH)). Tunapuna/Piarco Reg., Trinidad Isl., Saint Augustine, XI-1976, Malaise trap, F. D. Bennett (2 males, USNMENT00969599, 00969611 (BMNH)). Tunapuna/Piarco Reg., Trinidad Isl., wasteground, Saint Augustine, 14. VI.1976, J. S. Noyes (1 male, USNMENT00969796 (BMNH)). Tunapuna/ Piarco Reg., Trinidad Isl., wasteground, Saint Augustine, 21.VI.1976, J. S. Noyes (1 female, 1 male, USNMENT00969752-00969753 (BMNH)). Tunapuna/Piarco Reg., Trinidad Isl., wasteground, Saint Augustine, 25.VI.1976, J. S. Noyes (1 male, USNMENT00969674 $(\mathrm{BMNH})$ ). VENEZUELA: Carabobo St., San Esteban, XI-1939, sweeping, P. Anduze (1 female, USNMENT00977564 (USNM)).

Distribution.-Neotropical Region: Argentina, Bolivia, Brazil, Colombia, Costa Rica, Dominica, Ecuador, Guadeloupe, Mexico, Nicaragua, Panama, Paraguay, Peru, Saint Vincent and the Grenadines, Trinidad and Tobago and Venezuela.

Biology.-No host recorded.

Comments. - The degree of infuscation is somewhat variable, and looking at the wing against a light background helps to reveal this character.

\section{Zaeucoila johnsoni Buffington and Gallardo, new species}

urn:lsid:zoobank.org:act:B5F047AB9E5D-448A-A951-3BDBFDD6A28C http://bioguid.osu.edu/xbiod_concepts/ 409616

$$
\text { (Figs. 44-51) }
$$

Description.-Coloration with head, mesosoma, metasoma black to dark brown; legs with coxae and hind femora dark brown, rest yellow. Lateral margin of occiput defined by scalloped, sharp carina, with variable striae joining posterior margin of eye. Vertical carina adjacent to ventral margin of antennal socket present, leading to deep inner orbital groove. Malar space adjacent to anterior articulation of mandible with distinct conical protuberance, smooth. Malar sulcus present, composed of single line. Orbital furrows sharp, divergent groove running from antennal sockets to dorsal end of malar sulcus. Dorsal aspect of vertex smooth with a few short, scattered setae. Posterior aspect of vertex smooth with fine setae. Hair punctures on lateral aspect of vertex present, indistinct. Placoidal sensilla present on F 2-11. Pubescence on lateral surface of pronotum present, sparse, with distinct row behind pronotal plate. Ridges extending posteriorly from lateral margin of pronotal plate present, typically short, with single prominent ridge extending to anterior margin of mesoscutum. Sculpture on mesoscutum absent, with sparse long hairs. Median mesoscutal carina present, composed of anteriorly broad elevation, narrowing posteriorly, ending at posterior margin of mesoscutum. Anterior admedial lines present, in conjunction with mesoscutal carina. Lateroventral mesopleural carina present, marking abrupt change of slope of mesopectus, terminating anteriorly just ventral to large, oblique notch. Mesopleural triangle present, distinctly impressed with distinct dorsal and ventral border, with single, long seta. Dorsal surface of scutellum laterally with distinct ridges radiating from plate, posteriorly with deep fovea and ridges. Latero-ventral margin of scutellum posterior to auricula deeply foveate. Subalar area abruptly broadened anteriorly, with an indicated longitudinal division. Pubescence consisting of few hairs on posterior part of metaplueron, 

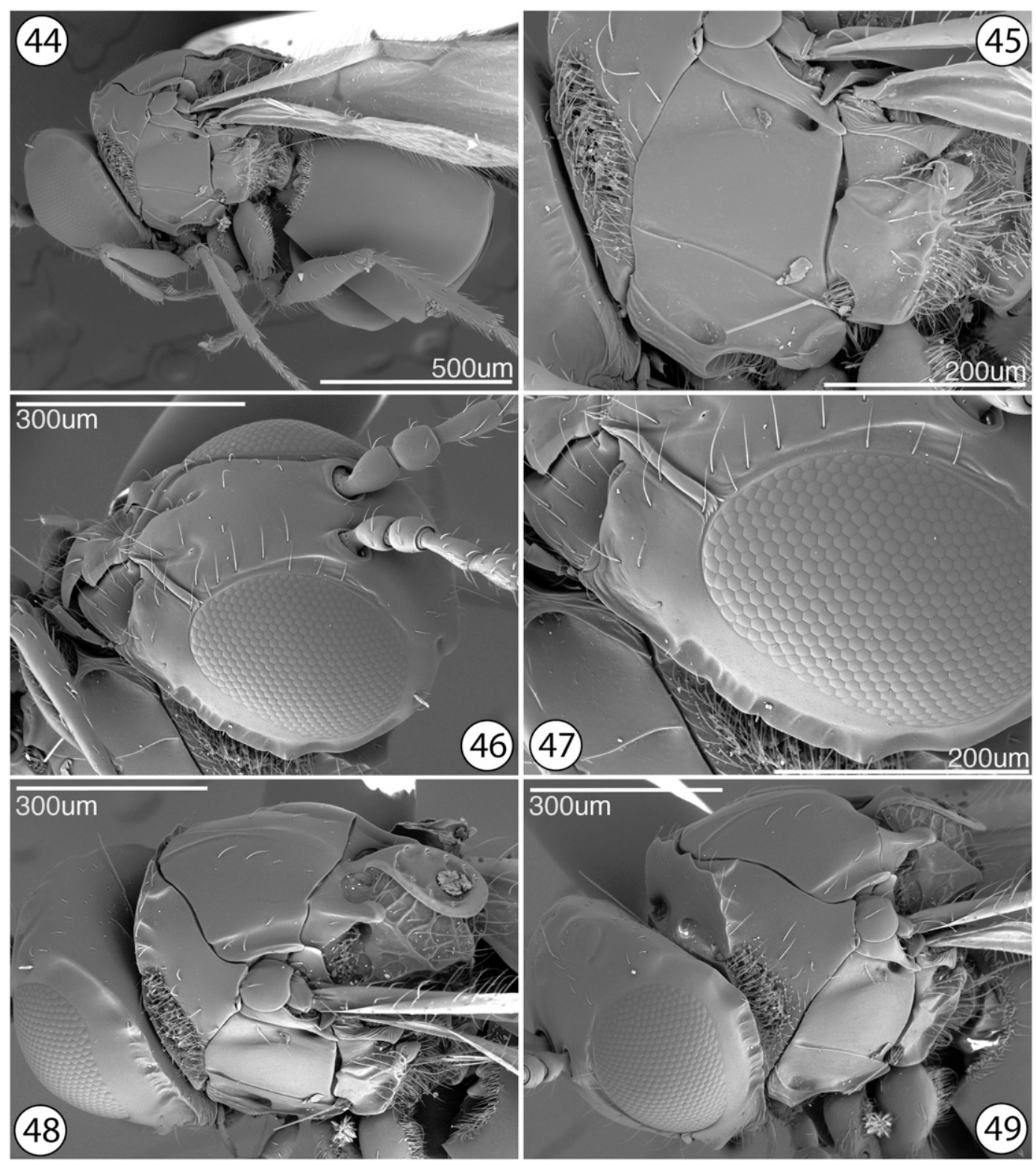

Plate 10, Figs. 44-49. Zaeucoila johnsonii, new species. 44, habitus, lateral view; 45, mesopleuron and metapleuron, lateral view; 46, head, anterolateral view; 47, head, lateral view; 48, head and mesosoma, dorsolateral view; 49, head and mesosoma, anterodorsal view.

dense hair on propodeum. Pubescence of fore wing mesally sparse, gradually becoming denser distally. Coloration of wing absent, entire wing hyaline. Hair fringe along apical margin of fore wing present, with particularly longer setae along the mesal anterior margin. Inter propodeal carinae space moderately setose, with long, thin setae evenly filling the space; surface underneath smooth. 

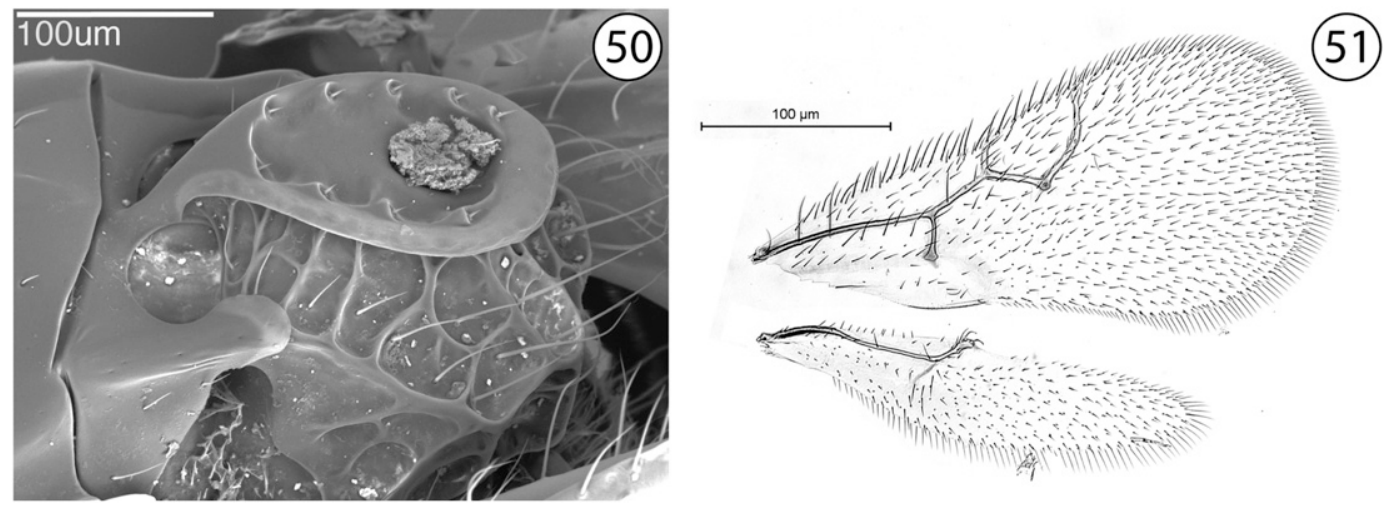

Plate 11, Figs. 50-51. Zaeucoila johnsonii, new species. 50, scutellum, laterodorsal view; 51, fore and hind wing.

Diagnosis._Zaeucoila johnsonii is morphologically most similar to Z. grenadensis. However, Z. johnsonii lacks the ocellar hair patches, and also lacks distinct parapsidal hair lines (though, trace tracks are still present). Zaeucoila johnsonii also has a very scalloped genal carina as well as a very simple, bold malar sulcus. While others species may have a scalloped genal carina, the very simple malar sulcus appears to be unique.

Etymology.-Named in honor of the eminent hymenopterist Dr. Norman Johnson (The Ohio State University). Dr. Johnson is a good friend of the first author, and has helped manage and format the data in this project for publication through vSysLab.

Link to Distribution Map.-[http:// hol.osu.edu/map-full.html id=409616]

Material Examined.-Holotype, male: COSTA RICA: Guanacaste Prov., H.4.C, Santa Rosa National Park, 300m, 11.V-1. VI.1985, Malaise trap, D. Janzen \& I. Gauld, USNMENT00977651 (deposited in USNM). Paratypes: (3 females, 3 males) COSTA RICA: Guanacaste Prov., H.4.C, Santa Rosa National Park, 300m, 11.V-1.VI.1985, Malaise trap, D. Janzen \& I. Gauld (1 female, 3 males,
USNMENT00977650, 00977652-00977654 (USNM)). Guanacaste Prov., SE.7.O1, Santa Rosa National Park, 300m, 1.V-22. V.1985, Malaise trap, D. Janzen \& I. Gauld (1 female, USNMENT00977659 (USNM)). Puntarenas Prov., Osa Peninsula, house of C. L. Madrigal, Puerto Jiménez, 10m, II1991, Malaise trap, P. Hanson (1 female, USNMENT01223593 (USNM)).

Distribution.-Neotropical Region: Costa Rica.

Biology._Unknown.

\section{Zaeucoila lignys Buffington and Gallardo, new species}

urn:1sid:zoobank.org:act:98549C1A86DA-470E-B836-C5470918E022 http://bioguid.osu.edu/xbiod_concepts/ 409606

(Figs. 52-57)

Description.-Coloration with head, mesosoma, metasoma black to dark brown; legs with coxae and hind femora dark brown, rest yellow. Lateral margin of occiput defined by evenly rounded, raised, sharp carina. Vertical carina adjacent to ventral margin of antennal socket present, leading to shallow inner orbital groove. Malar space adjacent to 

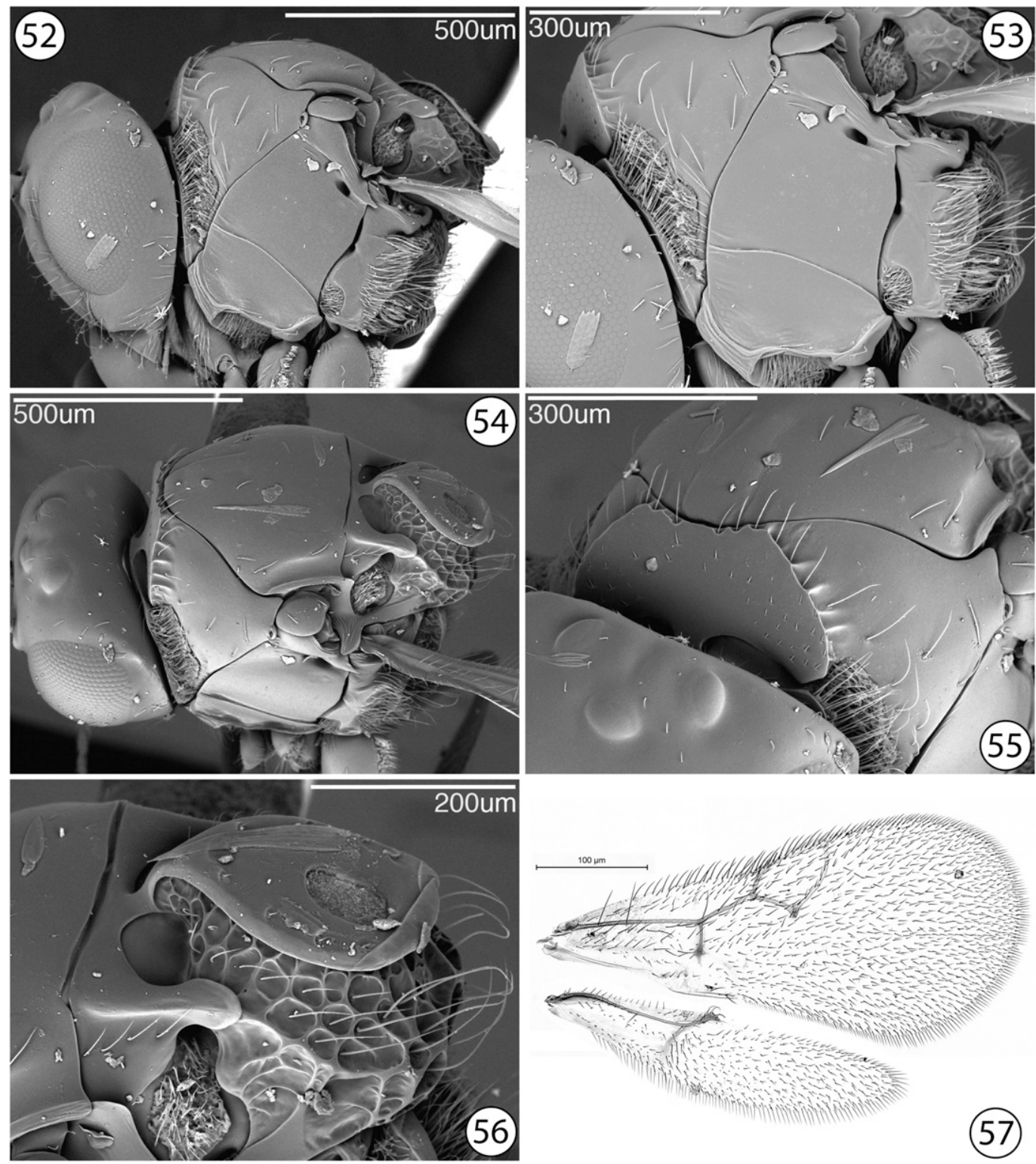

Plate 12, Figs. 52-57. Zaeucoila lignys, new species. 52, head and mesosoma, lateral view; 53, mesosoma, lateral view; 54, head and mesosoma, dorso-lateral view; 55, pronotal plate, antero-dorsal view; 56, scutellum, dorso-lateral view; 57, fore and hind wings.

anterior articulation of mandible with distinct pyramidal protuberance, smooth. Malar sulcus present, composed of series of small striae dorsally, leading to single line ventrally. Orbital furrows rounded, ill defined, running from lateral edge of torulus to ventral margin of compound eye. Dorsal aspect of vertex smooth with a few short, scattered setae. Posterior aspect of vertex smooth with fine setae. Hair punctures on lateral aspect of vertex absent. Placoidal sensilla present 
on F 2-11. Pubescence on lateral surface of pronotum present, sparse, with distinct row behind pronotal plate. Ridges extending posteriorly from lateral margin of pronotal plate present, variable, short to medium long, in some cases running to anterior margin of mesoscutum. Sculpture on mesoscutum absent, entire surface smooth, shiny. Median mesoscutal carina variable, present to absent; when present, merely indicated by an outline of the carina, never reaching midline of mesoscutum. Anterior admedial lines present, with adjacent cuticular surface smooth.

Lateroventral mesopleural carina present, marking abrupt change of slope of mesopectus, terminating anteriorly at anterior flange of mesosubpleuron. Mesopleural triangle present, gently impressed with distinct dorsal border, ventral border lacking; smooth. Dorsal surface of scutellum foveate-areolet. Latero-ventral margin of scutellum posterior to auricula deeply foveate. Subalar area slightly broadened anteriorly, without longitudinal division indicated. Pubescence present, medium density, along posterior and ventral margins of metapleuron; short and dense on propodeum. Pubescence of fore wing mesally sparse, gradually becoming denser distally. Coloration of wing variable mesally, ranging from very light blotchy infuscation to restricted infuscation immediately adjacent to the basalis the ventral bulb of basalts; rest of wing hyaline. Hair fringe along apical margin of fore wing present, with particularly longer setae along the mesal anterior margin. Inter propodeal carinae space densely setose, surface smooth.

Diagnosis.-This unusual species has the unique combination of a highly reduced to absent mesoscutal carina, and the possession of a very slight infuscation surrounding the posterior wing veins in the forewing. This latter character is shared with $Z$. unicarinata, but can be separated from this species by the lack of a mesoscutal carina (present in Z. unicarinata).

Etymology.-The name 'lignys' is Greek for smoke, in reference to the condition of wing membrane around and near the wing veins. The name is a noun in apposition.

Link to Distribution Map.-[http:// hol.osu.edu/map-full.html $\mathrm{id}=409606]$

Material Examined.-Holotype, female: GUADELOUPE: Basse-Terre Arrond., Capesterre-Belle-Eau, GP 2051, Grand Étang, 15.V.2005, reared, J. Etienne, USNMENT00977552 (deposited in USNM). Paratypes: (44 females, 24 males) ARGENTINA: Misiones Prov., nr. Loreto, wet forest, $77 \mathrm{~m}, 27.34^{\circ} \mathrm{S}$ $55.53^{\circ} \mathrm{W}$, Santa Ana, 27.III.2003, sweeping, J. Munro (1 female, USNMENT00993683 (USNM)). BRAZIL: SC, $27^{\circ} 11^{\prime} \mathrm{S} 52^{\circ} 23^{\prime} \mathrm{W}$, Nova Teutônia, 19.IX.1949, F. Plaumann (1 male, USNMENT00969873 (BMNH)). COLOMBIA: montane rainforest, $500 \mathrm{~m}$, 054ㄴ $\mathrm{N} 76^{\circ} 30^{\prime} \mathrm{W}$, Chocó Dept., 11.IV13.IV.1973 (1 female, USNMENT00977620 (USNM)). COSTA RICA: Cartago Prov., old field / agricultural area, Pejibaye, 24. III-25.III.1987, Malaise trap, W. Steiner (1 female, USNMENT01223594 (USNM)). Puntarenas Prov., $3 \mathrm{~km}$ SW Rincón, forest edge, Golfo Dulce Forest Reserve, 10m, VII-1991, Malaise trap, M. Pérez Parra (1 female, USNMENT00977790 (USNM)). Puntarenas Prov., Osa Peninsula, house of C. L. Madrigal, Puerto Jiménez, 10m, I-1991, Malaise trap (2 females, USNMENT00977833, 00977838 (USNM)). Puntarenas Prov., Osa Peninsula, house of C. L. Madrigal, Puerto Jiménez, 10m, II-1991, Malaise trap (1 female, USNMENT00977751 (USNM)). Puntarenas Prov., Osa Peninsula, house of C. L. Madrigal, Puerto Jiménez, 10m, III-1991, Malaise trap (1 female, USNMENT00977748 (USNM)). Puntarenas 
Prov., Puerto Jiménez, II-1991 (1 male, USNMENT00977737 (USNM)). ECUADOR: Napo Prov., 10km NE Tena, 400m, 19.II-20.II.1983, L. Masner (1 female, USNMENT00977693 (USNM)). Pichincha Prov., E of Puerto Quito, 2.II.1983, L. Huggert (1 female, USNMENT00977705 (USNM)). GUADELOUPE: Basse-Terre Arrond., Capesterre-Belle-Eau, GP 2051, Grand Étang, 15.V.2005, J. Etienne (5 females, USNMENT00977554-00977558 (USNM)). Basse-Terre Arrond., CapesterreBelle-Eau, GP 2051, Grand Étang, 15. V.2005, reared, J. Etienne (3 females, USNMENT00977550-00977551, 00977553 (USNM)). Basse-Terre Arrond., at feet of Morne Houël, GP 2193, Saint-Claude, 13.V.2004, reared, J. Etienne (1 female, 2 males, USNMENT00977924, 00977950, 00977952 (USNM)). GP 1060, Grand Étang, 7.II.1995, reared, J. Etienne (1 female, USNMENT00977929 (USNM)). MEXICO: CAM, $80 \mathrm{~km}$ W San Francisco de Campeche, 29.VII.1984, G. Gordh (1 female, USNMENT00977439 (UCRC)). TAMPS, $8.2 \mathrm{~km} \mathrm{E} \mathrm{Ciudad}$ Victoria, MEX-70, 3.VII.1986, G. Zolnerowich \& R. Trevino (1 female, USNMENT00977386 (TAMU)). TAMPS, 97km E Ciudad Victoria, MEX-70, 3. VII.1986, G. Zolnerowich \& R. Trevino (2 females, USNMENT00977369, 00977373 (TAMU)). SAINT VINCENT AND THE GRENADINES: Charlotte Parish, rainforest edge, Montreal, 8.VII.1976, J. S. Noyes (3 females, USNMENT0096969600969697, 00969701 (BMNH)). Charlotte Parish, rainforest, E slope of La Soufrière, 9.VII.1976 (1 female, USNMENT00969680 (BMNH)). TRINIDAD AND TOBAGO: Tobago Isl., Saint Patrick Parish, coastal grassland, Mount Irvine Bay, 19.VII.1976, J. S. Noyes (1 female, USNMENT00969541 (BMNH)). Trinidad Isl., Maracas Valley, II-1978, Malaise trap, F. D. Bennett (1 female, USNMENT00969621 (BMNH)). Trinidad
Isl., Maracas Valley, X-1977, Malaise trap, F. D. Bennett (1 female, 3 males, USNMENT00969606, 00969616, 00969623, 00969652 (BMNH)). Trinidad Isl., Maracas Valley, XII-1977, Malaise trap, F. D. Bennett (3 females, USNMENT00969601, 00969622, 00969653 (BMNH)). Tunapuna-Piarco Reg., Trinidad Isl., rainforest, Guanapo Valley, 29.VII.1976, J. S. Noyes (1 male, USNMENT00969689 (BMNH)). Tunapuna/Piarco Reg., Trinidad Isl., Saint Augustine, IX-1976, Malaise trap, F. D. Bennett (6 females, 10 males, USNMENT00969673, 00969706, 0096971200969714, 00969717, 00969719-00969720, 00969722, 00969726-00969727, 00969736, 00969738, 00969740, 00969745-00969746 (BMNH)). Tunapuna/Piarco Reg., Trinidad Isl., Saint Augustine, XI-1976, Malaise trap, F. D. Bennett ( 2 females, 4 males, USNMENT00969614, 00969617-00969618, 00969624, 00969628-00969629 (BMNH)). Tunapuna/Piarco Reg., Trinidad Isl., wasteground, Saint Augustine, 14. VI.1976, J. S. Noyes (2 females, 1 male, USNMENT00969798-00969800 (BMNH)). Tunapuna/Piarco Reg., Trinidad Isl., wasteground, Saint Augustine, 25. VI.1976, J. S. Noyes (1 male, USNMENT00969666 (BMNH)).

Distribution.-Neotropical region: Argentina, Brazil, Colombia, Costa Rica, Ecuador, Guadeloupe, Mexico, Saint Vincent and The Grenadines and Trinidad and Tobago.

Biology.-Host not recorded.

\section{Zaeucoila normae Gallardo and Buffington, new species}

urn:1sid:zoobank.org:act:E1794018B48E-4C1F-AE4E-7AEDE95BA25D http://bioguid.osu.edu/xbiod_concepts/ 409608

(Figs. 58-63) 

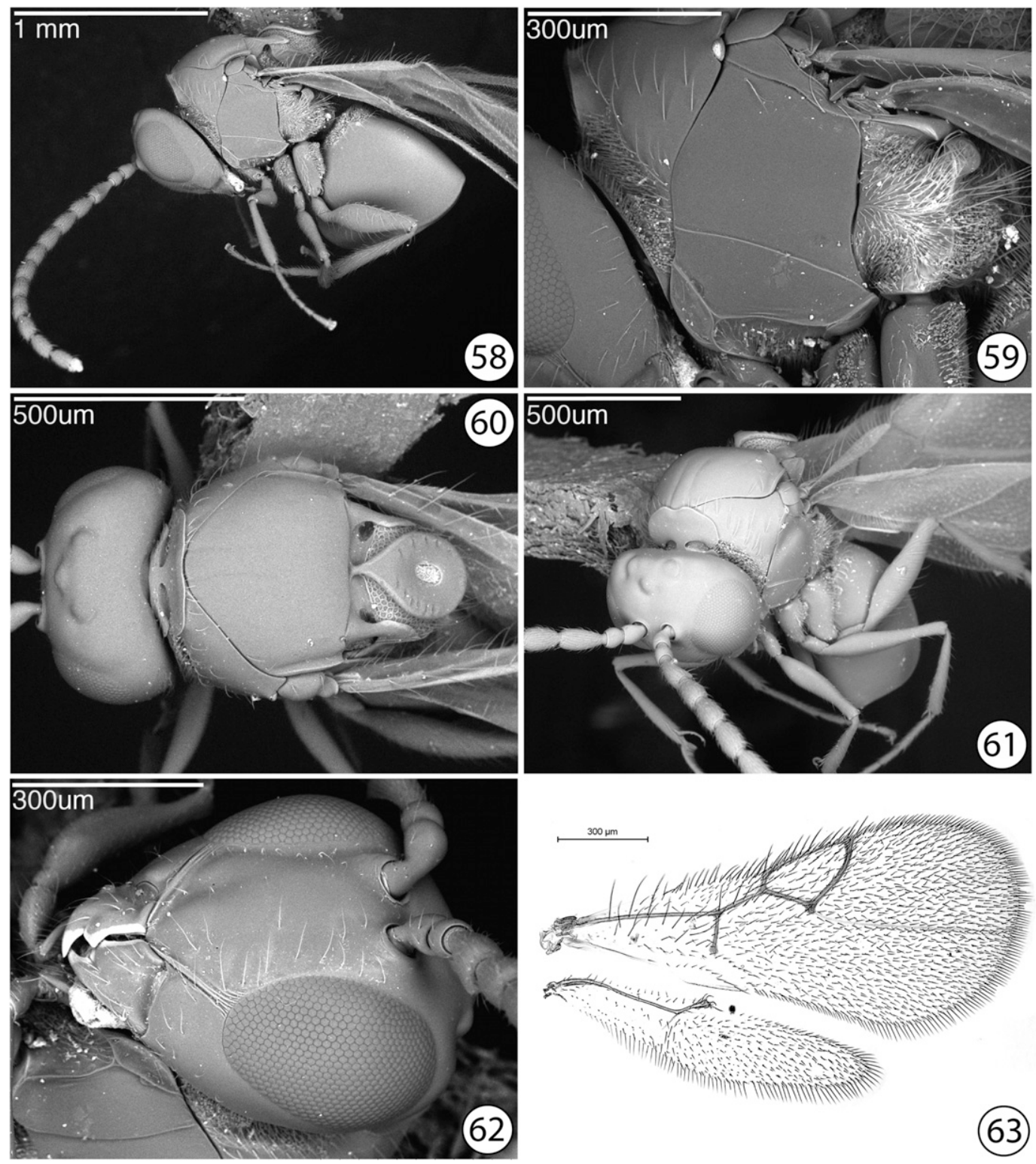

Plate 13, Figs. 58-63. Zaeucoila normae, new species. 58, habitus, lateral view; 59, mesopleuron and metapleuron, lateral view; 60, head and mesosoma, dorsal view; 61, habitus, antero-lateral view; 62, head, anterior view; 63, fore and hind wings.

Description.-Coloration with head, mesosoma, metasoma black to dark brown; legs yellow-orange. Lateral margin of occiput defined by evenly rounded, raised, sharp carina. Vertical carina adjacent to ventral margin of antennal socket absent. Malar space adjacent to anterior articulation of mandible with short, blunt, smooth protuberance. Malar sulcus present, composed of series of small striae dorsally, leading to single line ventrally. Orbital furrows absent. 
Dorsal aspect of vertex smooth. Posterior aspect of vertex smooth. Hair punctures on lateral aspect of vertex absent. Placoidal sensilla present on F211. Pubescence on lateral surface of pronotum present, sparse, with distinct row behind pronotal plate. Ridges extending posteriorly from lateral margin of pronotal plate absent. Sculpture on mesoscutum absent, entire surface smooth, shiny. Median mesoscutal carina absent. Anterior admedial lines present, with adjacent cuticular surface smooth. Lateroventral mesopleural carina present, marking abrupt change of slope of mesopectus, terminating anteriorly at large, oblique notch. Mesopleural triangle present, gently impressed with distinct dorsal border, ventral border lacking; smooth. Dorsal surface of scutellum foveate-areolet. Latero-ventral margin of scutellum posterior to auricula almost entirely smooth, weakly rugulose dorsally. Subalar area antero-ventrally broadened, excavated, median area smooth with setae. Pubescence long, dense, silvery on metapleuron; long, thin on propodeum. Pubescence of fore wing mesally sparse, gradually becoming denser distally. Coloration of wing absent, entire wing hyaline. Hair fringe along apical margin of fore wing present, with particularly longer setae along the mesal anterior margin. Inter propodeal carinae space moderately setose, with long, thin setae evenly filling the space; surface underneath smooth.

Diagnosis.-Wholly unique amongst Zaeucoila, and zaeucoilines in general, by having an entire setose metapleuron. At most, some species will have the latter third of the metapleuron setose, but never the entire sclerite as in Z. normae.

Etymology.-Named in honor of the eminent hymenopterist Dr. Norma Díaz of Museo de La Plata, Argentina. Dr. Díaz's research on Neotropical eucoiline wasps was ahead of its time in the $1970^{\prime} \mathrm{s}$ and 1980 's.

Link to Distribution Map.-[http:// hol.osu.edu/map-full.html?id=409608]

Material Examined.-Holotype, female: ARGENTINA: Buenos Aires Prov.,

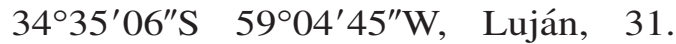
III.2005, Malaise trap, Coviella, USNMENT00993862 (deposited in USNM). Paratypes: (21 females, 9 males) ARGENTINA: Buenos Aires Prov.,

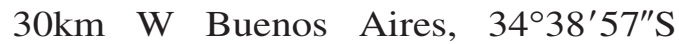
$58^{\circ} 46^{\prime} 57^{\prime \prime} \mathrm{W}, \quad$ Mariano Moreno, 16. II.2005, Malaise trap, Coviella (2 females, 1 male, USNMENT00993776, 00993858, 00993860 (USNM)). Buenos Aires Prov., 30km W Buenos Aires, $34^{\circ} 38^{\prime} 57^{\prime \prime} \mathrm{S} \quad 58^{\circ} 46^{\prime} 57^{\prime \prime} \mathrm{W}, \quad$ Mariano Moreno, 22.I.2005, Malaise trap, Coviella (1 female, USNMENT00993762 (USNM)). Buenos Aires Prov., 5mi E Berisso, nr. river, Paulino Island, 9. II.1997, screen sweeping, S. Triapitsyn (1 male, USNMENT00977463 (UCRC)). La Rioja Prov., Santa Cruz, 31.XII.2002, pan trap, P. Fidalgo (1 male, USNMENT00993706 (USNM)). Misiones

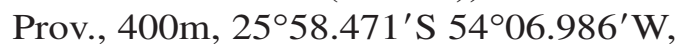
Vida Silvestre "Urugua-í" Wildlife Reserve, 7.XII-9.XII.2003, Malaise trap, B. V. Brown \& G. Kung (1 male, USNMENT00993844 (USNM)). Salta Prov., road to San Andrés, along Blanca River, $633 \mathrm{~m}, 23.13^{\circ} \mathrm{S} 63.48^{\circ} \mathrm{W}$, San Ramón de la Nueva Orán, 22.III.2003, sweeping, J. Munro (1 female, USNMENT00993772 (USNM)). Santiago del Estero Prov., 10km ENE Colonia Dora, Salado River, 17.XI-25.XI.1979, C. Vardy \& M. Vardy (1 female, USNMENT00970110 (BMNH)). Tucumán Prov., San Javier, 21.X.1950, Flezel (1 male, USNMENT01223497 (IMLA)). BRAZIL: GO, Itumbiara, 15. IV.1998, Marchiori (1 female, USNMENT01223498 (MLPA)). GO, Itumbiara, 6.VI.1998, Marchiori (1 female, USNMENT01223499 (MLPA)). RJ, Silva 
Jardim Mpio., Poço das Antas Biological Reserve, 1998, reared, Barbosa da Silva ( 2 males, USNMENT01223500-01223501 (MLPA)). RO, 62km S Ariquemes, nr. Cacaulândia, $10^{\circ} 32^{\prime} \mathrm{S} 62^{\circ} 48^{\prime} \mathrm{W}$, Rancho Grande Farm, 25.XII.1995, flight intercept trap, D. Kistner (1 female, KUNHM-ENT SM0135089 (SEMC)). SC, $27^{\circ} 11^{\prime} \mathrm{S} 52^{\circ} 23^{\prime} \mathrm{W}$, Nova Teutônia, 17.XII.1937, F. Plaumann (1 male, USNMENT00969787 (BMNH)). SC, $27^{\circ} 11^{\prime} \mathrm{S} 52^{\circ} 23^{\prime} \mathrm{W}$, Nova Teutônia, II-1937, F. Plaumann (1 female, USNMENT00969822 (BMNH)). COLOMBIA: Magdalena Dept., Neguanje, M.2230, $10 \mathrm{~m}, 11^{\circ} 20^{\prime} \mathrm{N} 74^{\circ} 02^{\prime} \mathrm{W}$, Tayrona Natural National Park, 18.X-31.X.2001, Malaise trap, R. Henriquez (1 female, USNMENT00993687 (USNM)). Magdalena Dept., Palangana, M.2227, 30m, $11^{\circ} 20^{\prime} \mathrm{N}$ $74^{\circ} 02^{\prime} \mathrm{W}$, Tayrona Natural National Park, 18.X-31.X.2001, Malaise trap, R. Henriquez (1 female, USNMENT00993871 (USNM)). Magdalena Dept., Tayrona Natural N.P., M.628, 30m, $11^{\circ} 20^{\prime} \mathrm{N}$

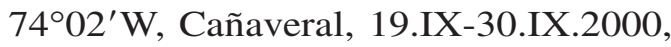
Malaise trap, R. Henriquez (1 male, USNMENT00993690 (USNM)). Vaupés Dept., Antigua Cabaña, M.3613, 60m, $01^{\circ} 04^{\prime} \mathrm{S} 69^{\circ} 31^{\prime} \mathrm{W}$, Mosiro Itajura-Caparú Biological Station, 10.III-17.IV.2003, Malaise trap, J. Pinzón (1 female, USNMENT00993689 (USNM)). COSTA RICA: Puntarenas Prov., Osa Peninsula, house of C. L. Madrigal, Puerto Jiménez, 10m, I-1991, Malaise trap (1 female, USNMENT00977834 (USNM)). Puntarenas Prov., Osa Peninsula, house of C. L. Madrigal, Puerto Jiménez, 10m, X-1991, Malaise trap (1 female, USNMENT00977755 (USNM)). Puntarenas Prov., Osa Peninsula, house of C. L. Madrigal, Puerto Jiménez, 10m, XII-1990, Malaise trap (1 female, USNMENT00993832 (USNM)). Puntarenas Prov., Puerto Jiménez, X-1990 (1 female, USNMENT00993771 (USNM)). ECUADOR:
Napo Prov., 10km NE Tena, 400m, 19.II20.II.1983, L. Masner (1 female, USNMENT00993692 (USNM)). Pichincha Prov., E of Puerto Quito, 2.II.1983, L. Huggert (2 females, USNMENT0099369400993695 (USNM)). Sucumbíos Prov., Sacha Lodge, 4.V-14.V.1994, P. Hibbs (1 female, USNMENT00993710 (USNM)). PERU: Yahuarmayo (Quebrada Yahuarmayo), 11.II.1910, C. H. T. Townsend (1 female, USNMENT00993944 (USNM)).

Distribution.-Neotropical region: Argentina, Brazil, Colombia, Costa Rica, Ecuador and Peru.

Biology.-Host not recorded.

\section{Zaeucoila patera Buffington and Gallardo, new species}

urn:1sid:zoobank.org:act:FBCA09FFD2A6-4347-B593-934E375D6EC5 http://bioguid.osu.edu/xbiod_concepts/ 408249

$$
\text { (Figs. 64-69) }
$$

Description.-Coloration with head, mesosoma, metasoma black to dark brown; legs with coxae and femora dark brown, rest yellow. Lateral margin of occiput defined by evenly rounded, raised, sharp carina. Vertical carina adjacent to ventral margin of antennal socket present, leading to shallow inner orbital groove. Malar space adjacent to anterior articulation of mandible with short, blunt, smooth protuberance. Malar sulcus present, composed of series of small striae dorsally, leading to single line ventrally. Orbital furrows rounded, divergent ledges running from antennal sockets to dorsal end of malar sulcus. Dorsal aspect of vertex smooth with a few short, scattered setae. Posterior aspect of vertex smooth. Hair punctures on lateral aspect of vertex absent. Placoidal sensilla present on F 2-11. Pubescence on lateral surface of pronotum 
present, sparse, with distinct row behind pronotal plate. Ridges extending posteriorly from lateral margin of pronotal plate absent. Sculpture on mesoscutum absent, entire surface smooth, shiny. Median mesoscutal carina absent. Anterior admedial lines absent. Lateroventral mesopleural carina present, marking abrupt change of slope of mesopectus, terminating anteriorly at anterior flange of mesosubpleuron. Mesopleural triangle present, distinctly impressed with distinct dorsal and ventral border, with single, long seta. Dorsal surface of scutellum irregularly rugulose. Latero-ventral margin of scutellum posterior to auricula smooth, with weak longitudinal striae. Subalar area slightly broadened anteriorly, with an indicated longitudinal division. Pubescence consisting of few hairs on posterior part of metaplueron, dense hair on propodeum. Pubescence of fore wing mesally sparse, gradually becoming denser distally. Coloration of wing absent, entire wing hyaline. Hair fringe along apical margin of fore wing present, with particularly longer setae along the mesal anterior margin. Inter propodeal carinae space moderately setose, with long, thin setae evenly filling the space; surface underneath smooth.

Diagnosis.-Zaeucoila patera is unique among the species of Zaeucoila in the morphology of the scutellar plate when viewed laterally. In all other Zaeucoila, the disk of the scutellum is raised distinction above the surface of the mesoscutum; in the case of the $Z$. patera, the disk of the scutellum is distinctly (albeit, slightly) below the surface of the mesoscutum.

Etymology.-Latin for saucer or plate, in reference to the very flat scutellar plate in lateral view. The name is a noun in apposition.

Link to Distribution Map.-[http:// hol.osu.edu/map-full.html?id=408249]
Material Examined.-Holotype, male: COSTA RICA: Guanacaste Prov., H.3.O, Santa Rosa National Park, 300m, 14.VIII6.IX.1985, Malaise trap, D. Janzen \& I. Gauld, USNMENT00993567 (deposited in USNM). Paratype: COSTA RICA: Guanacaste Prov., H.3.O, Santa Rosa National Park, 300m, 14.VIII-6.IX.1985, Malaise trap, D. Janzen \& I. Gauld (1 male, USNMENT00993572 (USNM)).

Distribution.-Neotropical region: Costa Rica.

Biology.-Host not recorded.

\section{Zaeucoila robusta (Ashmead), new combination}

http://bioguid.osu.edu/xbiod_concepts/ 408976

(Figs. 70-75)

Chrestosema robusta Ashmead, 1894: 68 (original description).

Agrostocynips robusta (Ashmead): Buffington, 2004: 10 (generic transfer); Buffington and Scheffer, 2008: 43 (description, diagnosis, type information); Buffington, 2009: 172 (listed); Forshage, Nordlander and Buffington, 2013: 247 (cataloged, type information).

Agrostocynips clavatus Díaz, 1976: 32 (in De Santis et. al. 1976) (original description), new synonym.

Zaeucoila enneatoma Díaz, 1975: 1999. new synonym.

Agrostocynips enneatoma (Díaz): Díaz and Gallardo, 1997: 39 (generic transfer)

Ganaspis diastrophi Ashmead 1896: 184185. new synonym.

Agrostocynips diastrophi (Ashmead): Buffington 2004:10 (generic transfer); Forshage et al., 2012 (catalogued).

Redescription.-Coloration with head, mesosoma, metasoma black to dark brown; legs variable, ranging from all leg segments entirely orange-yellow to coxae and femora dark brown, rest yellow. Lateral margin of occiput with distinct sharp 
carina ventrally, running midway to eye; dorsally absent, margin evenly rounded. Vertical carina adjacent to ventral margin of antennal socket absent. Malar space adjacent to anterior articulation of mandible with distinct pyramidal protuberance, smooth. Malar sulcus present, composed of series of small striae dorsally, leading to single line ventrally. Orbital furrows absent. Dorsal aspect of vertex smooth with a few short, scattered setae. Posterior aspect of vertex smooth with fine setae. Hair
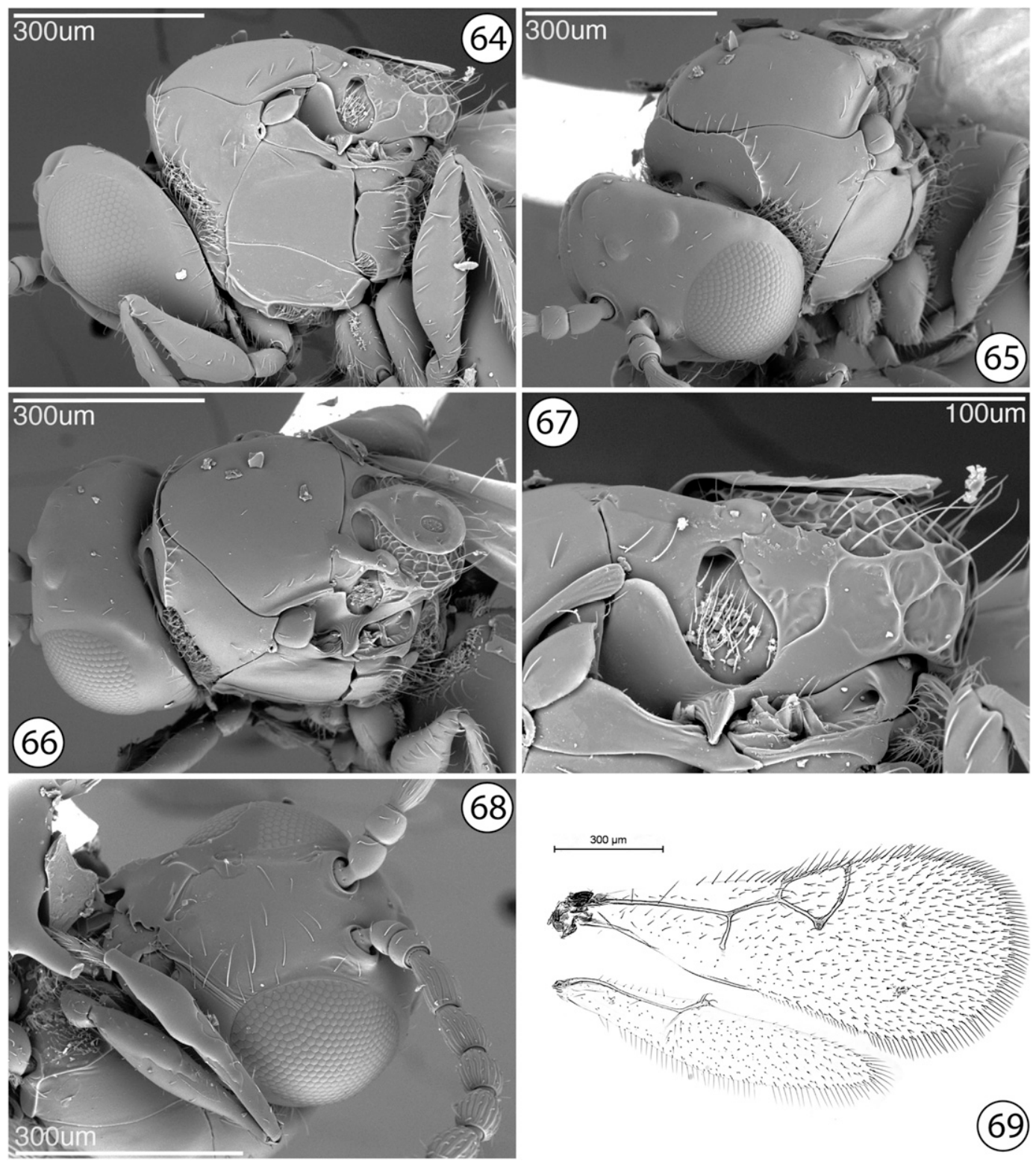

(69)

Plate 14, Figs. 64-69. Zaeucoila patera, new species. 64, head and mesosoma, lateral view; 65, head and mesosoma, antero-dorsal view; 66, head and mesosoma, dorso-lateral view; 67, scutellum, lateral view; 68, head, antero-lateral view; 69, fore and hind wings. 

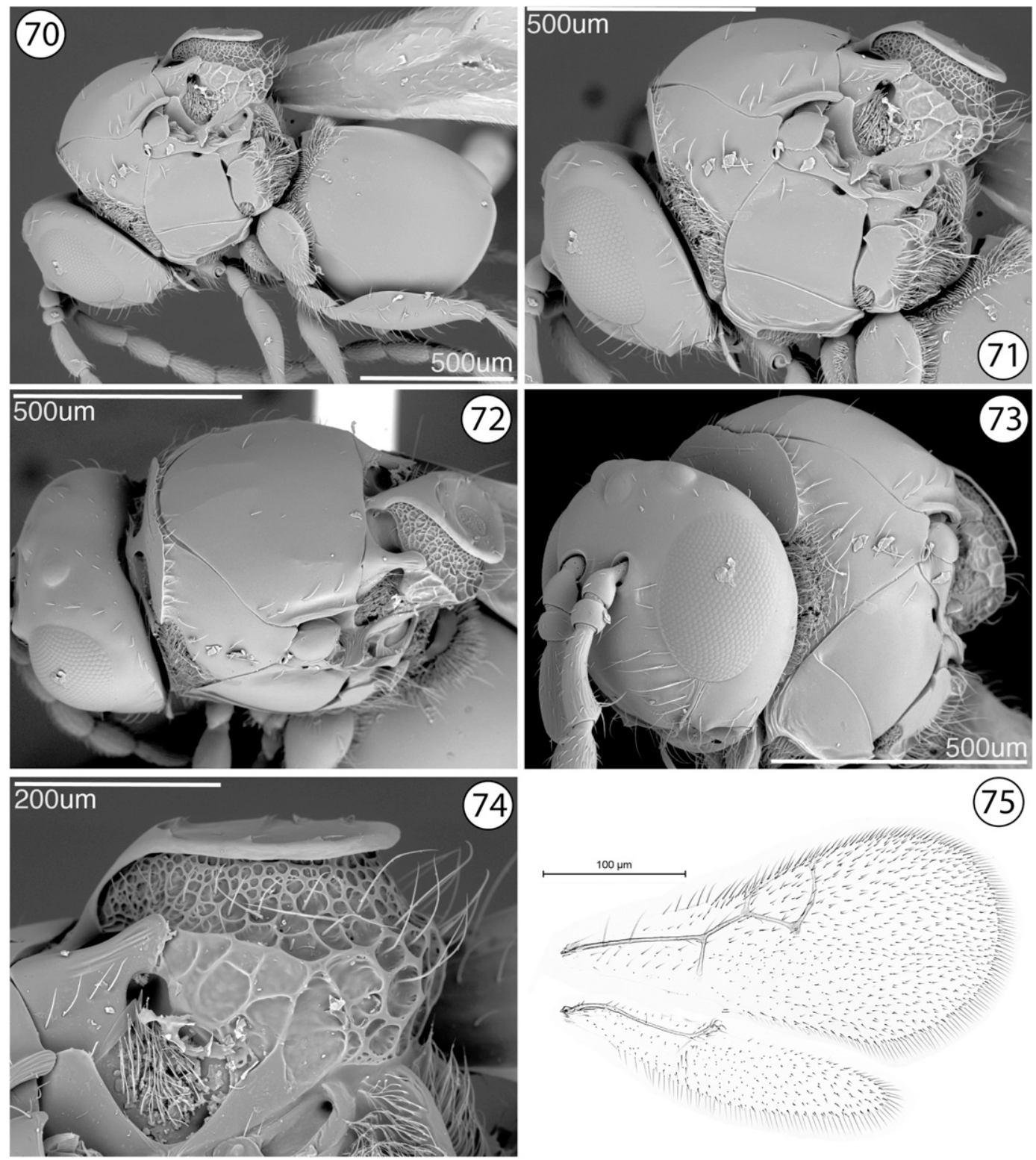

Plate 13, Figs. 70-75. Zaeucoila robusta (Ashmead). 70, habitus, lateral view; 71, head and mesosoma, lateral view; 72, head and mesosoma, dorso-lateral view; 73, head and mesosoma, antero-lateral view; 74, scutellum, lateral view; 75, fore and hind wings.

punctures on lateral aspect of vertex absent. Placoidal sensilla present on F 2-11. Pubescence on lateral surface of pronotum present, sparse, with distinct row behind pronotal plate. Ridges extending posteriorly from lateral margin of pronotal plate distinct but short, not extending to the dorsal margin of pronotum. Sculpture on mesoscutum absent, entire surface smooth, shiny. Median mesoscutal carina variable, present to absent; when present, merely indicated by an outline of the carina, never 
reaching midline of mesoscutum. Anterior admedial lines present, with adjacent cuticular surface smooth. Lateroventral mesopleural carina present, marking abrupt change of slope of mesopectus, terminating anteriorly at anterior flange of mesosubpleuron. Mesopleural triangle present, gently impressed with distinct dorsal border, ventral border lacking; smooth. Dorsal surface of scutellum foveateareolet. Latero-ventral margin of scutellum posterior to auricula deeply foveate. Subalar area slightly broadened anteriorly, with an indicated longitudinal division. Pubescence present, but very sparse, along posterior and ventral margins of metapleuron; short and dense on propodeum. Pubescence of fore wing mesally sparse, gradually becoming denser distally. Coloration of wing absent, entire wing hyaline. Hair fringe along apical margin of fore wing present, with particularly longer setae along the mesal anterior margin. Inter propodeal carinae space smooth with confined setal patch.

Diagnosis.-Zaeucoila robusta is the only known species in the genus with an incomplete genal carina. With a specimen in lateral view, the carina is visible from the posterior articulation of the mandible to the midpoint of the compound eye. In all other species, the carina continues clearly beyond the dorsal margin of the eye.

Link to Distribution Map.-[http:// hol.osu.edu/map-full.html?id=408976]

Material Examined. Type specimens: Chrestosema robusta: [first label] St. Vincent, W[est] I[ndies], H.H. Smith, [second label] Type No. 2336 USNM [third label] Chrestosem robusta Ashm. [in Ashmead's hand], [fourth label ] Agrostocynips robusta (Ahm.), Det. By M.L. Buffington 2003. Agrostocynips clavatus: Argentina, Güemes (Provinicia de Salta), 1930, Ogloblin, col., MLP. Ganaspis diastrophi: [first label] West
Point, Neb[raska], [second label, folded] Diastrophus cuscataiformis [in Ashmead's hand], [third label] Type No. 3280 U.S.N.M., [fourth label] Ganaspis diastro- phi Ashmead, type [in Ashmead's hand]. Other material: (748 females, 655 males, 27 unknowns) ANGUILLA: 12.II19.III.1982, A. G. Parker (1 female, USNMENT00970045 (BMNH)). 20.III23.IV.1982, A. G. Parker (1 female, 1 male, USNMENT00970044, 00970049 (BMNH)). 28.V.1982, A. G. Parker (3 females, USNMENT00970046-00970048 (BMNH)). ARGENTINA: Buenos Aires Prov., 30km W Buenos Aires, 34³8'57"S $58^{\circ} 46^{\prime} 57^{\prime \prime} \mathrm{W}, \quad$ Mariano Moreno, 16. II.2005, Malaise trap, Coviella (1 female, USNMENT00993766 (USNM)). Buenos Aires Prov., $30 \mathrm{~km}$ W Buenos Aires, 34 $38^{\prime} 57^{\prime \prime} \mathrm{S} \quad 58^{\circ} 46^{\prime} 57^{\prime \prime} \mathrm{W}$, Mariano Moreno, 22.I.2005, Malaise trap, Coviella (2 females, USNMENT0099376300993764 (USNM)). Buenos Aires Prov.,

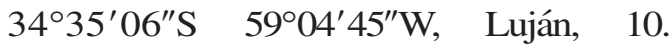
III.2005, Malaise trap, Coviella (1 male, USNMENT00993868 (USNM)). Buenos Aires Prov., $34^{\circ} 35^{\prime} 06^{\prime \prime} \mathrm{S} 59^{\circ} 04^{\prime} 45^{\prime \prime} \mathrm{W}$, Luján, 31.III.2005, Malaise trap, Coviella (6 males, USNMENT00993861, 00993863-00993867 (USNM)). Buenos Aires Prov., $\mathrm{N}$ of Buenos Aires, $34^{\circ} 23^{\prime} 50^{\prime \prime} \mathrm{S} 58^{\circ} 34^{\prime} 32^{\prime \prime} \mathrm{W}$, Tigre, 2.I-11. I.2006, Malaise trap, Logarzo (2 females, USNMENT00993852-00993853 (USNM)). Buenos Aires Prov., $\mathrm{N}$ of Buenos Aires, 34 $23^{\circ} 50^{\prime \prime} \mathrm{S} 58^{\circ} 34^{\prime} 32^{\prime \prime} \mathrm{W}$, Tigre, 23.II-5.III.2006, Malaise trap, Logarzo (1 female, 2 males, USNMENT00993849-00993851 (USNM)). Buenos Aires Prov., N of Buenos Aires, $34^{\circ} 23^{\prime} 50^{\prime \prime} \mathrm{S} 58^{\circ} 34^{\prime} 32^{\prime \prime} \mathrm{W}$, Tigre, 5.III12.III.2006, Malaise trap, Logarzo (2 females, USNMENT00993854-00993855 (USNM)). Catamarca Prov., nr. La Merced, roadside scrub / Acacia, $869 \mathrm{~m}, 28.20^{\circ} \mathrm{S}$ $65.64^{\circ} \mathrm{W}$, RN 38, 18.III.2003, sweeping, J. Munro (1 female, USNMENT00993757 
(USNM)). La Rioja Prov., 1757m, $28^{\circ} 53^{\prime} 40^{\prime \prime} \mathrm{S} 67^{\circ} 00^{\prime} 31^{\prime \prime} \mathrm{W}$, Chuquis, 17. III.2003, sweeping, J. Munro (1 female, USNMENT00993756 (USNM)). La Rioja Prov., El Duraznillo, 1.I-15.I.2003, Malaise trap, P. Fidalgo (2 females, 2 males, USNMENT00993696-00993699 (USNM)). La Rioja Prov., Santa Cruz, 1.XII-15.XII.2002, Malaise trap, P. Fidalgo (2 females, 1 male, USNMENT00993846-00993848 (USNM)). La Rioja Prov., Santa Cruz, 15.IX-14. X.2002, Fidalgo (20 females, 11 males, 2 unknowns, USNMENT0099371200993715, 00993717-00993724, 00993726, 00993730-00993732, 00993734-00993739, 00993741-00993751 (USNM)). 1999, N. Díaz (17 females, 5 males, USNMENT00977466-00977468, 0097747000977472, 00977474-00977488, 00977490 (UCRC)). Buenos Aires Prov., 3435'06"S 5904'45"W, Luján, 31.III.2006, Malaise trap, Coviella (1 male, USNMENT01223604 (USNM)). Buenos Aires Prov., La Plata, 29.XII.1970, Díaz \& Loiácono (1 female, USNMENT01223502 (MLPA)). Buenos Aires Prov., nr. Otamendi, 34 $10^{\prime} 52^{\prime \prime S} 58^{\circ} 52^{\prime} 12^{\prime \prime} \mathrm{W}$, Paraná River, 23.I.2003, S. Triapitsyn (1 female, USNMENT00977506 (UCRC)). Buenos Aires Prov., $\sim 5 \mathrm{mi}$ E Berisso, nr. river, Paulino Island, 9.II.1997, screen sweeping, S. Triapitsyn (1 male, USNMENT00977462 (UCRC)). Catamarca Prov., $\sim 2 \mathrm{~km}$ N Santa Maria, $\sim 1800 \mathrm{~m}$, 21.III.1974, Malaise trap, C. R. Vardy (1 female, USNMENT00969636 (BMNH)). Córdoba Prov., 10.XI.1978, Valladares (1 female, USNMENT01223505 (MLPA)). Córdoba Prov., 20.XII-26.XII.1978, Valladares (4 females, USNMENT0122350601223509 (MLPA)). Córdoba Prov., 6.II-7. II.1978 (2 females, USNMENT0122350301223504 (MLPA)). Jujuy Prov., XII-1978, Román (1 female, USNMENT01223510 (MLPA)). La Rioja Prov., Castro Barros Dept., La Calera, 17.XI.2001, P. Fidalgo
(1 female, USNMENT00977465 (UCRC)). La Rioja Prov., Santa Cruz, 15.IX-14. X.2002, Malaise trap, Fidalgo (1 female, USNMENT00977976 (USNM)). La Rioja Prov., Santa Cruz, 31.XII.2002, pan trap, P. Fidalgo (3 females, 4 males, USNMENT00993700-00993703, 00993705, 00993707, 00993775 (USNM)). Mendoza Prov., Potrerillos, 1700m, 8.I.1980, Fidalgo (1 male, USNMENT01223511 (MLPA)). Misiones Prov., 200m, $25.68^{\circ} \mathrm{S} 54.45^{\circ} \mathrm{W}$, Iguazú National Park, no date (1 female, USNMENT00993708 (USNM)). Misiones Prov., Loreto, 5.IV.1931 (1 female, USNMENT01223512 (MLPA)). Misiones Prov., Loreto, III-1931 (1 male, USNMENT01223514 (MLPA)). Misiones Prov., Loreto, no date (1 female, 1 male, USNMENT01223513, 01223515 (MLPA)). Misiones Prov., nr. Loreto, wet forest, $77 \mathrm{~m}, 27.34^{\circ} \mathrm{S} 55.53^{\circ} \mathrm{W}$, Santa Ana, 27.III.2003, sweeping, J. Munro (1 female, USNMENT00993769 (USNM)). Neuquén Prov., 40 41'23.5'S $71^{\circ} 17^{\prime} 33.2^{\prime \prime} \mathrm{W}$, Nahuel Huapi National Park, 20.II-6.III.2008, Malaise trap, Garré \& Montes de Oca (3 females, 2 males, USNMENT01223520-01223524 (MLPA)). Neuquén Prov., 40 41'23.5"S $71^{\circ} 17^{\prime} 33.2^{\prime \prime} \mathrm{W}$, Nahuel Huapi National Park, 7.II-20.II.2008, Malaise trap, Garré \& Montes de Oca (4 females, USNMENT01223516-01223519 (MLPA)). Salta Prov., 66km E jct. RN-34, dry chaco, JM22/H24, $23^{\circ} 14^{\prime} 07^{\prime \prime} \mathrm{S} 63^{\circ} 23^{\prime} 22^{\prime \prime} \mathrm{W}, \mathrm{RN}$ 81, 24.III.2003, sweeping, J. Munro (1 male, USNMENT00993758 (USNM)).

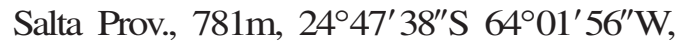
Cabeza de Buey, 21.III.2003, sweeping, J. Munro (1 male, USNMENT00993761 (USNM)). Salta Prov., Hotel Termas grounds, $899 \mathrm{~m}, 25^{\circ} 50^{\prime} 14^{\prime \prime} \mathrm{S} 64^{\circ} 55^{\prime} 55^{\prime \prime} \mathrm{W}$, Rosario de la Frontera, 22.III.2003, sweeping, J. Munro (1 female, USNMENT00993774 (USNM)). Salta Prov., Rosario de Lerma, 16.XII-18.XII.1983, 
Malaise trap, M. Wasbauer (1 female, USNMENT01081051 (USNM)). Salta Prov., Rosario de la Frontera Dept., Juramento River, $730 \mathrm{~m}, 24.79^{\circ} \mathrm{S} 65.03^{\circ} \mathrm{W}$, Lumbrera, 21.III.2003, sweeping, J. Munro ( 1 female, 1 male, USNMENT0099375900993760 (USNM)). Santiago del Estero Prov., 10km ENE Colonia Dora, Salado River, 17.XI-25.XI.1979, C. Vardy \& M. Vardy (17 females, 13 males, USNMENT00970077-00970079, 00970082-00970083, 00970085, 00970087, 00970089-00970092, 00970095-00970096, 00970099-00970103, 00970105, 00970109, 00970112, 00970114, 00970117-00970124 (BMNH)). 1999, N. Díaz (9 females, 6 males, USNMENT00977087-00977101 (USNM)). So. Amer. Paras. Lab No 9702, Buenos Aires Prov., 8.XI.1944, Parker (1 female, USNMENT00977022 (USNM)). Tucumán Prov., VI-1976, Fidalgo (2 females, USNMENT01223525-01223526 (MLPA)). Tucumán Prov., VIII-1978, Fidalgo (2 males, USNMENT0122352701223528 (MLPA)). Tucumán Prov., herbaceous vegetation, San Javier, 16. XI.1981, Mulvany, Díaz et al. (14 females, 12 males, USNMENT0122352901223554 (MLPA)). Tucumán Prov., herbaceous vegetation, Tafí del Valle, 17.XI.1981, Mulvany, Armesto et al. (5 females, 1 male, USNMENT0122355501223560 (MLPA)). BOLIVIA: Santa Cruz Dept., $18^{\circ} 06^{\prime} \mathrm{S} 63^{\circ} 36^{\prime} \mathrm{W}$, El Refugio Los Volcanes, 25.X-30.X.2007, Malaise trap, J. Romero \& F. Romero (1 male, USNMENT00977969 (USNM)). BRAZIL: BA, Centro de Pesquisas do Cacau (CEPEC), Itabuna, 24.II-27. II.1983, F. P. Benton (1 female, USNMENT00969748 (BMNH)). BA, Centro de Pesquisas do Cacau (CEPEC), Itabuna, X-1978, F. P. Benton (1 female, USNMENT00969660 (BMNH)). GO, 17. I.1998 (1 female, USNMENT01223562 (MLPA)). GO, 9.VI.1998 (1 female, USNMENT01223561 (MLPA)). MG,
Araporã, 19.IX.2002, Marchiori (1 female, USNMENT01223563 (MLPA)). RJ, Silva Jardim Mpio., Poço das Antas Biological Reserve, 1998, reared, Barbosa da Silva (1 female, USNMENT01223564 (MLPA)). SC, 27 $11^{\prime}$ S $52^{\circ} 23^{\prime} \mathrm{W}$, Nova Teutônia, 15.XII.1938, F. Plaumann (2 females, 1 male, USNMENT00969781, 00969882, 00969902 (BMNH)). SC, $27^{\circ} 11^{\prime} \mathrm{S} 52^{\circ} 23^{\prime} \mathrm{W}$, Nova Teutônia, 17.VI.1937, F. Plaumann (1 female, USNMENT00969867 (BMNH)). SC, $27^{\circ} 11^{\prime} \mathrm{S} 52^{\circ} 23^{\prime} \mathrm{W}$, Nova Teutônia, 17.XI.1938, F. Plaumann (1 male, USNMENT00969864 (BMNH)). SC, $27^{\circ} 11^{\prime} \mathrm{S} 52^{\circ} 23^{\prime} \mathrm{W}$, Nova Teutônia, 18. XI.1938, F. Plaumann (1 female, USNMENT00969894 (BMNH)). SC, 2711'S $52^{\circ} 23^{\prime} \mathrm{W}$, Nova Teutônia, 29.V.1937, F. Plaumann (1 female, USNMENT00969773 (BMNH)). SC, $27^{\circ} 11^{\prime} \mathrm{S} 52^{\circ} 23^{\prime} \mathrm{W}$, Nova Teutônia, 6.II.1937, F. Plaumann (1 male, USNMENT00969886 (BMNH)). SC, $27^{\circ} 11^{\prime} \mathrm{S} 52^{\circ} 23^{\prime} \mathrm{W}$, Nova Teutônia, 8.VIII.1935, F. Plaumann (1 male, USNMENT00969821 (BMNH)). SC, $27^{\circ} 11^{\prime} \mathrm{S} 52^{\circ} 23^{\prime} \mathrm{W}$, Nova Teutônia, 9. IX.1949, F. Plaumann (1 female, USNMENT00969907 (BMNH)). SC, 27¹1'S $52^{\circ} 23^{\prime} \mathrm{W}$, Nova Teutônia, II-1937, F. Plaumann (2 females, 1 male, USNMENT00969835, 00969841, 00969843 $(\mathrm{BMNH})) . \quad \mathrm{SC}, 27^{\circ} \mathrm{S} 52.58^{\circ} \mathrm{W}$, Nova Teutônia, 24.III.1938, F. Plaumann (1 female, USNMENT00970010 (BMNH)). SC, Nova Teutônia, 13.VII.1943, F. Plaumann (1 male, USNMENT00969586 (BMNH)). SP, Itirapina, 15.XII.1992, F. Ejchel (1 female, USNMENT01223565 (MZSP)). SP, São Paulo, 9.X.1957, K. A. Spencer (1 male, USNMENT00969904 $(\mathrm{BMNH})$ ). CANADA: ON, 5km NW Kemptville, 19.IV-25.IV.1990, D. Masner (1 male, USNMENT01081076 (USNM)). ON, Toronto, 1897, C. F. Baker (1 male, USNMENT00977010 (USNM)). ON, University of Guelph Arboretum, Guelph, 
5.VI.2006, Malaise trap, L. Coote (1 male, USNMENT00993685 (USNM)). CHILE: Arica and Parinacota Reg., Arica Prov., Azapa, VIII-1965, Vargas (6 females, USNMENT01223566-01223571 (MLPA)). Arica and Parinacota Reg., Arica Prov., Azapa, VIII-1967 (1 female, USNMENT00977008 (USNM)). BíoBío Reg., Nuble Prov., 15km E San Carlos, 23.XII.1950, Ross \& Michelbacher (1 female, USNMENT00977459 (CAS)). Bío-Bío Reg., Ñuble Prov., $18 \mathrm{~km}$ E San Carlos, 24.XII.1950, Ross \& Michelbacher (1 female, USNMENT00977460 (CAS)). Valparaíso Reg., valley, Llaillay (Llay-Llay), 2. XII.1958, De Santis (1 female, USNMENT01223572 (MLPA)). no date (2 females, USNMENT00977018-00977019 (USNM)). dry scrub, $32^{\circ} 35.975^{\prime} \mathrm{S}$ $71^{\circ} 14.369^{\prime} \mathrm{W}$, no date, screen sweeping, $\mathrm{J}$. George (1 female, USNMENT00993784 (USNM)). COLOMBIA: Bolívar Dept., Alto el Mirador, M.1602, 400m, $09^{\circ} 54^{\prime} \mathrm{N} 75^{\circ} 07^{\prime} \mathrm{W}$, Los Colorados Fauna and Flora Sanctuary, 6.IV-21.IV.2001, Malaise trap, E. Deulufeut (5 males, USNMENT00977873-00977876, 01223605 (USNM)). Bolívar Dept., Alto el Mirador, M.1605, 400m, $09^{\circ} 54^{\prime} \mathrm{N}$ $75^{\circ} 07^{\prime} \mathrm{W}$, Los Colorados Fauna and Flora Sanctuary, 22.IV-7.V.2001, Malaise trap, E. Deulufeut (1 male, USNMENT00977870 (USNM)). Bolívar Dept., Alto el Mirador, M.1724, 400m, $09^{\circ} 54^{\prime} \mathrm{N} 75^{\circ} 07^{\prime} \mathrm{W}$, Los Colorados Fauna and Flora Sanctuary, 7.V-22.V.2001, Malaise trap, E. Deulufeut (1 female, USNMENT00977877 (USNM)). Bolívar Dept., Alto el Mirador, M.1725, 400m, $09^{\circ} 54^{\prime} \mathrm{N} 75^{\circ} 07^{\prime} \mathrm{W}$, Los Colorados Fauna and Flora Sanctuary, 22.V-7. VI.2001, Malaise trap, E. Deulufeut (2 males, USNMENT00977871-00977872 (USNM)). Bolívar Dept., Villa Roca, M.1603, 180m, 09 $54^{\prime} \mathrm{N} 75^{\circ} 07^{\prime} \mathrm{W}$, Los Colorados Fauna and Flora Sanctuary,
22.IV-7.V.2001, Malaise trap, E. Deulufeut (1 male, USNMENT00993777 (USNM)). Magdalena Dept., Palangana, M.2134, 30m, $11^{\circ} 20^{\prime} \mathrm{N} 74^{\circ} 02^{\prime} \mathrm{W}$, Tayrona Natural National Park, 9.IX-17. IX.2001, Malaise trap, R. Henriquez (1 female, USNMENT00993688 (USNM)). Magdalena Dept., Zaino, M.789, 50m, $11^{\circ} 20^{\prime} \mathrm{N} 74^{\circ} 02^{\prime} \mathrm{W}$, Tayrona Natural National Park, 10.X-3.XI.2000, Malaise trap, R. Henriquez (1 female, 2 males, USNMENT00977903-00977905 (USNM)). Magdalena Dept., Zaino, M.793, 50m, $11^{\circ} 20^{\prime} \mathrm{N} 74^{\circ} 02^{\prime} \mathrm{W}$, Tayrona Natural National Park, 29.IX-17.X.2000, Malaise trap, R. Henriquez ( 2 females, 5 males, USNMENT00977823, 00977825-00977828, 00977830-00977831 (USNM)). Magdalena Dept., Zaino, M.967, 50m, $11^{\circ} 20^{\prime} \mathrm{N}$ $74^{\circ} 02^{\prime} \mathrm{W}$, Tayrona Natural National Park, 15.XII-2.I.2001, Malaise trap, R. Henriquez (1 female, 1 male, USNMENT00977211-00977212 (USNM)). Tolima Dept., Fresno Mun., Vda. Colombia, M.111, $1508 \mathrm{~m}, 5^{\circ} 12^{\prime} \mathrm{N} 75^{\circ} 02^{\prime} \mathrm{W}$, Las Perlas Farm, 23.XII-26.XII.2002, Malaise trap, T. Arias, D. Arias \& S. Arias (1 unknown, USNMENT00993872 (USNM)). Valle del Cauca Dept., Anchicaya, M.1110, 730m, $03^{\circ} 26^{\prime} \mathrm{N} 76^{\circ} 48^{\prime} \mathrm{W}$, Farallones de Cali Natural National Park, 25.X-8.XI.2000, Malaise trap, S. Sarria (6 males, USNMENT00977213, 0097787900977883 (USNM)). COSTA RICA: Alajuela Prov., Peninsula Path, 600m, $10^{\circ} 27^{\prime} \mathrm{N} 84^{\circ} 40^{\prime} \mathrm{W}$, Arenal Volcano National Park, 25.II.2003, J. Noyes (1 female, USNMENT00993831 (USNM)). Cacao, I-1990 - II-1990 (1 female, USNMENT00993770 (USNM)). Cartago Prov., Linda Vista Nursery, Dulce Nombre, 1300m, VI-1993 - VIII-1993 (1 female, USNMENT00977810 (USNM)). Cartago Prov., Pejibaye, 24.III-25.III.1987, W. Steiner (1 female, USNMENT01223603 (USNM)). Guanacaste Prov., $13 \mathrm{~km} \mathrm{E}$ Filadelfia, 40m, V-1989 - VI-1989, 
Malaise trap, M. Garcia (1 female, USNMENT00993823 (USNM)). Guanacaste Prov., $13 \mathrm{~km}$ SE La Cruz, El Hacha Hill, 300m, no date, Janzen \& Gauld (3 females, 1 male, USNMENT00993825-00993828 (USNM)). Guanacaste Prov., 13km SE La Cruz, El Hacha Hill, 300m, no date, D. Janzen \& I. Gauld (1 male, USNMENT00977974 (USNM)). Guanacaste Prov., 20km SW Cañas, Enrique Jimenez Nuñez Experimental Station, 5.XI-17.XI.1991, Malaise trap, A. S. Menke (1 female, 5 males, USNMENT00993946-00993950, 00993954 (USNM)). Guanacaste Prov., Cacao Hill, Mengo Station, Guanacaste National Park, 1000m, V-1988, Gauld \& Jansen (1 female, 2 males, USNMENT00969932, 00969980-00969981 (BMNH)). Guanacaste Prov., H.1.O, Santa Rosa National Park, 300m, 10.I-31.I.1987, Malaise trap, D. Janzen \& I. Gauld (2 females, USNMENT00977991-00977992 (USNM)). Guanacaste Prov., H.1.O, Santa Rosa National Park, 300m, 14.VI-5.VII.1985, Malaise trap, D. Janzen \& I. Gauld (1 female, USNMENT00993578 (USNM)). Guanacaste Prov., H.1.O, Santa Rosa National Park, 300m, 18.X-18.XI.1986, Malaise trap, D. Janzen \& I. Gauld (1 female, 1 male, USNMENT01029047, 01029053 (USNM)). Guanacaste Prov., H.1.O, Santa Rosa National Park, 300m, 5.VII-26.VII.1986, Malaise trap, D. Janzen \& I. Gauld (1 female, USNMENT01029078 (USNM)). Guanacaste Prov., H.1.O, Santa Rosa National Park, 300m, 6.IX-27.IX.1986, Malaise trap, D. Janzen \& I. Gauld (1 male, USNMENT00993588 (USNM)). Guanacaste Prov., H.1.O, Santa Rosa National Park, 300m, 8.XI-29.XI.1986, Malaise trap, D. Janzen \& I. Gauld (1 unknown, USNMENT01029062 (USNM)). Guanacaste Prov., H.2.C, Santa Rosa National Park, 300m, 1.VI-27.VI.1985, Malaise trap, D. Janzen \& I. Gauld (1 female,
USNMENT00993589 (USNM)). Guanacaste Prov., H.2.C, Santa Rosa National Park, 300m, 13.IV-4.V.1986, Malaise trap, D. Janzen \& I. Gauld (1 male, USNMENT01029028 (USNM)). Guanacaste Prov., H.2.C, Santa Rosa National Park, 300m, 14.VIII-6.IX.1986, Malaise trap, D. Janzen \& I. Gauld (1 unknown, USNMENT01029020 (USNM)). Guanacaste Prov., H.2.C, Santa Rosa National Park, 300m, 19.X-8.XI.1986, Malaise trap, D. Janzen \& I. Gauld (1 female, USNMENT00977981 (USNM)). Guanacaste Prov., H.2.C, Santa Rosa National Park, 300m, 20.XII-10.I.1987, Malaise trap, D. Janzen \& I. Gauld (1 female, USNMENT00977988 (USNM)). Guanacaste Prov., H.2.C, Santa Rosa National Park, 300m, 8.XI-29.XI.1986, Malaise trap, D. Janzen \& I. Gauld (2 females, USNMENT01029087-01029088 (USNM)). Guanacaste Prov., H.3.O, Santa Rosa National Park, 300m, 11.V-1. VI.1985, Malaise trap, D. Janzen \& I. Gauld (2 females, 1 male, USNMENT01029114-01029116 (USNM)). Guanacaste Prov., H.3.O, Santa Rosa National Park, 300m, 13.VII-3.VIII.1985, Malaise trap, D. Janzen \& I. Gauld (4 females, USNMENT01029094-01029097 (USNM)). Guanacaste Prov., H.3.O, Santa Rosa National Park, 300m, 14.VI-5.VII.1986, Malaise trap, D. Janzen \& I. Gauld (1 female, USNMENT00993573 (USNM)). Guanacaste Prov., H.3.O, Santa Rosa National Park, 300m, 18.X-8.XI.1986, Malaise trap, D. Janzen \& I. Gauld ( 2 females, 1 male, USNMENT01029106, 01029109-01029110 (USNM)). Guanacaste Prov., H.3.O, Santa Rosa National Park, 300m, 21.II14.III.1987, Malaise trap, D. Janzen \& I. Gauld (2 females, USNMENT0102909101029092 (USNM)). Guanacaste Prov., H.3.O, Santa Rosa National Park, 300m, 26.VII-14.VIII.1986, Malaise trap, D. Janzen \& I. Gauld (1 female, 1 unknown, 
USNMENT00993585, 00993662 (USNM)). Guanacaste Prov., H.3.O, Santa Rosa National Park, 300m, 27.IX-18.X.1985, Malaise trap, D. Janzen \& I. Gauld (1 female, USNMENT01223602 (USNM)). Guanacaste Prov., H.3.O, Santa Rosa National Park, 300m, 27.IX-18.X.1986, Malaise trap, D. Janzen \& I. Gauld (1 female, USNMENT00993600 (USNM)). Guanacaste Prov., H.3.O, Santa Rosa National Park, 300m, 5.VII-26. VII.1986, Malaise trap, D. Janzen \& I. Gauld (2 females, 2 males, USNMENT00993595-00993598 (USNM)). Guanacaste Prov., H.3.O, Santa Rosa National Park, 300m, 6.IX-27.IX.1986, Malaise trap, D. Janzen \& I. Gauld (5 females, USNMENT01029082-01029086 (USNM)). Guanacaste Prov., H.3.O, Santa Rosa National Park, 300m, 8.XI29.XI.1986, Malaise trap, D. Janzen \& I. Gauld (2 females, USNMENT0102907901029080 (USNM)). Guanacaste Prov., H.4.C, Santa Rosa National Park, 300m, 1.VI-22.VI.1985, Malaise trap, D. Janzen \& I. Gauld (1 female, USNMENT01029061 (USNM)). Guanacaste Prov., H.4.C, Santa Rosa National Park, 300m, 6.IX-27.IX.1986, Malaise trap, D. Janzen \& I. Gauld (2 females, USNMENT01029005-01029006 (USNM)). Guanacaste Prov., H.4.C, Santa Rosa National Park, 300m, 8.XI-29.XI.1986, Malaise trap, D. Janzen \& I. Gauld (1 female, USNMENT00977985 (USNM)). Guanacaste Prov., SE.6.C, Santa Rosa National Park, 300m, 13.VII-3.VIII.1985, Malaise trap, D. Janzen \& I. Gauld (1 female, 1 male, USNMENT0102911701029118 (USNM)). Guanacaste Prov., SE.7.O, Santa Rosa National Park, 300m, 14.VI-5.VII.1986, Malaise trap, D. Janzen \& I. Gauld (1 female, USNMENT01029111 (USNM)). Guanacaste Prov., SE.8.C, Santa Rosa National Park, 300m, 21.II-14.III.1987, Malaise trap, D. Janzen \& I. Gauld (1 female, USN-
MENT01029089 (USNM)). San José Prov., Escazú Canton, San Antonio de Escazú, 1300m, VI-1988, Gauld (1 female, USNMENT00969946 (BMNH)). CUBA: Camagüey Prov., grass, Camagüey, 3.XII.1957 (1 female, USNMENT00993993 (USNM)). Pinar del Río Prov., San Vicente, 1955 , N. L. H. Krauss (1 female, USNMENT00977009 (USNM)). DOMINICA: Saint John Parish, Portsmouth, 0-100m, VII-1979, N. L. H. Krauss (1 female, USNMENT00977852 (USNM)). Saint Joseph Parish, Grande Savane, 8. IX.1965, D. L. Jackson (1 female, USNMENT00993785 (USNM)). Saint Paul Parish, site 1, Mount Joy, 5.VI-8. VI.1991, Malaise trap, H. Geitzenauer (1 female, USNMENTO0977419 (TAMU)). ECUADOR: Napo Prov., 10km NE Tena, 400m, 19.II-20.II.1983, L. Masner (1 female, USNMENT00993691 (USNM)). Sucumbíos Prov., 00³0'S 76³0' W, Sacha Lodge, 4.V-14.V.1994, P. Hibbs (1 female, USNMENT00993709 (USNM)). GUADELOUPE: Basse-Terre Arrond., Bouillante Commune, route de Damba, GP 2108, Pigeon, 19.VIII.2003, J. Etienne (2 females, USNMENT00993782-00993783 (USNM)). Basse-Terre Arrond., CapesterreBelle-Eau, GP 2312, Grand Étang, 16. IX.2005, reared, J. Etienne (2 females, USNMENT00977774, 00977777 (USNM)). Basse-Terre Arrond., GP 162, VieuxHabitants, 24.XII.1985, reared, J. Etienne (5 females, USNMENT0097793600977940 (USNM)). Basse-Terre Arrond., Lamentin, GP 1822, Ravine Chaude, 26. IV.2000, reared, J. Etienne (3 females, USNMENT00977771-00977773 (USNM)). Basse-Terre Arrond., Les Amandiers, GP 1056, Sainte-Rose, 17.I.1995, reared, J. Etienne (4 females, USNMENT00977955-00977958 (USNM)). Basse-Terre Arrond., Saint Louis, GP 2317, Bailiff, 26.X.2005, reared, J. Etienne (3 females, USNMENT00977765, 00977779-00977780 (USNM)). Pointe- 
à-Pitre Arrond., GP 1045, Pointe des Châteaux, 3.I.1995, reared, J. Etienne (2 females, USNMENT00977953-00977954 (USNM)). Pointe-à-Pitre Arrond., GP 511, Petit-Canal, 6.XII.1990, reared, J. Etienne ( 2 females, USNMENT0097791600977917 (USNM)). Pointe-à-Pitre Arrond., Les Abymes, 21.III.1986, reared, Miath (5 females, USNMENT00977918-00977919, 00977923, 00977925-00977926 (USNM)). GUATEMALA: Sacatepéquez Dept., Antigua Guatemala, X-1965, N. L. H. Krauss (1 male, USNMENT00993926 (USNM)). Sacatepéquez Dept., Sumpango, $14^{\circ} 40^{\prime} 17^{\prime \prime} \mathrm{N} \quad 90^{\circ} 43^{\prime} 11^{\prime \prime} \mathrm{W}$, Durwest Farm, 17.III-20.III.2007, M. Hoddle (1 male, USNMENT00993836 (USNM)). JAMAICA: Portland Parish, Manchioneal, 30.I.1937, Chapin \& Blackwelder (1 female, USNMENT00993920 (USNM)). Saint Thomas Parish, Bath, 6.II.1937, Chapin \& Blackwelder (2 females, USNMENT00993921-00993922 (USNM)). MARTINIQUE: Fort-de-France Arrond., MP 073, Saint-Joseph, 5.VIII.1996, reared, J. Etienne (5 females, USNMENT00977784-00977788 (USNM)). Marin Arrond., M 20, Le François, 6. VIII.1986, reared, Mezen (4 females, 2 males, USNMENT00977907-00977910, 00977915, 00977921 (USNM)). Marin Arrond., M 25, Sainte-Anne, 16.X.1986, reared, Mezen (3 females, USNMENT00977947-00977949 (USNM)). Trinité Arrond., MP 071, Morne des Esses, 5.VIII.1996, reared, J. Etienne (3 females, USNMENT00977781-00977783 (USNM)). Trinité Arrond., MP 49, L'Ajoupa-Bouillon, 26.IV.1994, reared, J. Etienne (5 females, USNMENT0097791100977914, 00977922 (USNM)). MEXICO: AGS, $0.25 \mathrm{mi}$ S southern fork MEX-70, Calvillo, 20.VII-8.VIII.2002, Malaise trap, M. Provance (4 males, USNMENT00977516-00977518, 00977525 (UCRC)). BCS, Las Barracas, 16.
XII.1984, pan trap, P. DeBach (2 females, USNMENT00977523-00977524 (UCRC)). BCS, Las Barracas, 18. XI.1984, P. DeBach (1 female, USNMENT00977507 (UCRC)). JAL, Zapopan, IX-1965, N. L. H. Krauss (1 male, USNMENT00977863 (USNM)). MICH, 100km N Lázaro Cárdenas, 7.VIII.1984, G. Gordh (1 male, USNMENT00977522 (UCRC)). NL, 8km N Ciudad General Terán, 16.V.1984, Gordh, Sierra \& Rodriguez (1 female, USNMENT00977510 (UCRC)). NL, San Juan River, San Juan, 14.VII.1983, A. Gonzalez H. (1 female, USNMENT00977508 (UCRC)). QRO, Jalpan River, $750 \mathrm{~m}, \quad 21^{\circ} 12^{\prime} 47^{\prime \prime} \mathrm{N}$ 99 28'09"W, Jalpan de Serra, 14. IV.2002, D. Yanega (1 female, USNMENT00993779 (USNM)). QROO, N of Punta Allen, dune scrub, Sian $\mathrm{Ka}^{\prime}$ an Biosphere Reserve, 3.VII.2004, screen sweeping, J. George \& C. George (1 female, 3 males, USNMENT00977980, 00993957-00993959 (USNM)). SIN, $11 \mathrm{mi} \mathrm{N}$ La Concha, nr. microwave station, La Muralla, 25.X.1982, J. T. Huber (1 female, USNMENT00977511 (UCRC)). SIN, El Chino de los López, VIII-1987, reared (1 female, 1 male, USNMENT00977519-00977520 (UCRC)). VER, 20 $0^{\circ} 11^{\prime} 20^{\prime \prime} \mathrm{N} 96^{\circ} 51^{\prime} 57^{\prime \prime} \mathrm{W}$, San Raphael Jicaltepec, no date (1 female, USNMENT00993935 (USNM)). VER, 97/015, 0.7mi N Jilotepec, 3680ft, 14. VI.1997, screen sweeping, L. A. Wilson \& J. B. Woolley (2 females, 1 male, USNMENT00977413-00977414, 00977417 (TAMU)). VER, Orizaba, no date, Crawford (1 male, USNMENT00993927 (USNM)). PANAMA: Panamá Oeste Prov., Canal Zone, Barro Colorado Island, 9.VII.1923, R. C. Shannon (1 female, USNMENT00653490 (USNM)). PERU: Callao Reg., Callao, 17.XI.1950, E. S. Ross \& A. E. Michelbacher (1 female, USNMENT00977452 (CAS)). Callao Reg., agricultural area, Callao, 
16.XI.1950, sweeping, Michelbacher \& Ross (1 female, USNMENT00977453 (CAS)). Cusco Reg., Machupicchu (Machu Picchu), 1385m, 11.VIII-14. VIII.1971, C. Vardy \& M. Vardy (1 female, USNMENT00970030 (BMNH)). Cuzco, Pisac, 3000m, 15.VIII.1971, C. Vardy \& M. Vardy (1 female, USNMENT00970029 (BMNH)). Lima Prov., Lima, 1970, M. Delgado (1 female, USNMENT00977007 (USNM)). Lima Prov., Lima, 4.X.1964 (1 female, USNMENT00977006 (USNM)). Lima Prov., Lima, no date (1 female, 2 males, USNMENT00993923-00993925 (USNM)). Lima Prov., Lima, no date, Delgado (2 females, 1 male, USNMENT0099399600993998 (USNM)). Lima Reg., Chancay River Valley, 15.III.1951, Michelbacher \& Ross (1 female, USNMENT00977454 (CAS)). Lima Reg., Chosica, XI-1961, N. L. H. Krauss (1 male, USNMENT00993936 (USNM)). Lima Reg., San Vicente de Cañete, 10.VIII.1964, J. E. Simone (1 male, USNMENT00993994 (USNM)). Lima Reg., San Vicente de Cañete, 19.V.1941, P. A. Berry (2 females, 2 males, USNMENT0097701200977015 (USNM)). Lima Reg., San Vicente de Cañete, 1965, Herrera (1 female, USNMENT00993995 (USNM)). SAINT KITTS AND NEVIS: Nevis Island, IX-1943, R. C. Fennah (1 female, USNMENT00653598 (USNM)). SAINT VINCENT AND THE GRENADINES: Charlotte Parish, rainforest edge, Montreal, 8.VII.1976, J. S. Noyes (1 female, USNMENT00969699 (BMNH)). Charlotte Parish, rainforest, E slope of La Soufrière, 9.VII.1976 (1 female, USNMENT00969554 (BMNH)). Saint David Parish, coastland, Richmond Beach, 10. VII.1976, J. S. Noyes (1 female, USNMENT00969679 (BMNH)). Saint George Parish, Belmont, 6.VII.1976, J. S. Noyes (1 female, USNMENT00969694 (BMNH)). Saint Vincent Island, no date, H. H.
Smith (1 female, USNMENT00977299 (USNM)). TRINIDAD AND TOBAGO: Diego Martin Reg., Trinidad Isl., secondary forest, Point Gourde, 8.VIII.1976, J. S. Noyes (1 female, USNMENT00969663 (BMNH)). San Fernando City, rice field, San Fernando, 18.X.1918, H. Morrison (1 female, USNMENT00993991 (USNM)). Tobago Isl., Saint Patrick Parish, coastal grassland, Mount Irvine Bay, 19. VII.1976, J. S. Noyes (1 female, USNMENT00969542 (BMNH)). Tobago Isl., neglected citrus orchard next to primary forest, Goldsborough, 24.II-3.III.1994, Malaise trap, M. J. Sommeijer (1 male, USNMENT00977851 (USNM)). Tobago Isl., rainforest edge, Parlatuvier Valley, 20.VII.1976, J. S. Noyes (1 female, USNMENT00969538 (BMNH)). UNITED STATES: AL, Covington Co., Salt Pond area, 98-12 / 98-13, Conecuh National Forest, 10.I.1998, reared, S. J. Scheffer (2 females, 2 males, USNMENT00977270-00977273 (USNM)). AL, Covington Co., Salt Pond, 98-13, Conecuh National Forest, 10.I.1998, reared, S. Scheffer ( 2 females, 3 males, USNMENT00977261-00977265 (USNM)). AL, Covington Co., nr. Salt Pond, 98-12, Conecuh National Forest, 10.I.1998, reared, S. Scheffer (1 female, 3 males, USNMENT00977266-00977269 (USNM)). AR, Polk Co., Queen Wilhelmina State Park, 4.VI.1987, G. F. Hevel \& J. F. Hevel (1 male, USNMENT00977357 (USNM)). AZ, Pima Co., Tucson, 27. V.1896, C. F. Baker \& R. E. Kunze (1 female, USNMENT00977011 (USNM)). AZ, Santa Cruz Co., Nogales, no date (1 male, USNMENT00977312 (USNM)). CA, San Diego Co., Pala, 25.V.1938, Malaise trap (1 unknown, USNMENT00993565 (USNM)). CO, Larimer Co., Fort Collins, no date (1 male, USNMENT00977328 (USNM)). FL, Alachua Co., American Entomological Institute (AEI), mixed deciduous forest, 
$29^{\circ} 35^{\prime} 53.6^{\prime \prime} \mathrm{N} \quad 82^{\circ} 21^{\prime} 54.8^{\prime \prime} \mathrm{W}$, Gainesville, III-2005, Malaise trap, D. B. Wahl ( 2 males, USNMENT00993666, 00993682 (USNM)). FL, Alachua Co., Gainesville, 29.IV.1937, L. J. Bottimer (1 female, USNMENT00993320 (USNM)). FL, Highlands Co., Price Tract, 98-21, Archbold Biological Station, 15.I.1998, reared, S. Scheffer (1 male, USNMENT00977298 (USNM)). FL, Jefferson Co., University of Florida NFRECMonticello, Monticello, 16.IV.2001, R. Mizell (1 female, USNMENT00977503 (UCRC)). FL, Miami-Dade Co., 21st Street, beach dune / coastal strand, $25^{\circ} 48.039^{\prime} \mathrm{N} \quad 80^{\circ} 07.509^{\prime} \mathrm{W}, \quad$ Miami Beach, 28.I-31.I.2009, yellow pan trap, H. Clebsch (1 female, USNMENT01223606 (USNM)). FL, Monroe Co., Key Largo, near Pole 90, $25^{\circ} 10.531^{\prime} \mathrm{N} 80^{\circ} 22.062 \mathrm{~W}$, Dagny Johnson Key Largo Hammock Botanical State Park, 16.IV.2010, M. L. Buffington (1 female, 1 male, USNMENT0099336100993362 (USNM)). FL, Polk Co., Lakeland, no date, reared, G. G. Ainslie ( 2 females, 2 males, USNMENT0097732200977325 (USNM)). FL, Wakulla Co., road nr. Crawfordville, 98-14, Apalachicola National Forest, 13.I.1998, reared, S. Scheffer (5 females, 5 males, USNMENT00977274-00977283 (USNM)). FL, Wakulla Co., road nr. Crawfordville, 98-14, Apalachicola National Forest, 13. I.1998, reared, S. J. Scheffer (2 females, 4 males, USNMENT00977292-00977293, 00977295-00977297, 00977303 (USNM)). FL, Wakulla Co., road nr. Crawfordville, 98-15, Apalachicola National Forest, 13. I.1998, reared, S. Scheffer ( 8 females, 2 males, USNMENT00977501-00977502 (UCRC); USNMENT00977284-00977291 (USNM)). GA, Bartow Co., open field, Cartersville, 27.VI.1986, J. D. Pinto (1 male, USNMENT00977513 (UCRC)). GA, Gordon Co., Fairmount, 34 $24^{\prime} 12^{\prime \prime} \mathrm{N}$ $84^{\circ} 46^{\prime} 08^{\prime \prime} \mathrm{W}$, Salacoa Creek, 16.V.2002,
Malaise trap, D. Yanega (1 female, USNMENT00977512 (UCRC)). Hardy Co., 11.VIII-28.VIII.2006 (1 female, USNMENT00993669 (USNM)). IA, Woodbury Co., South Ravine, mixed vegetation, Sioux City, no date, sweeping, C. N. Ainslie (2 females, USNMENT00977335, 00977354 (USNM)). IL, Cook Co., Evanston, 15.VII.1917 (1 male, USNMENT00977349 (USNM)). IL, Effingham Co., SSW of Mason, 7. IX.1993, J. D. Pinto (1 female, USNMENT00977515 (UCRC)). IL, Greene Co., Carrollton, 14.V.1991, screen sweeping, H. E. Andersen (1 male, USNMENT00977492 (UCRC)). IL, Marion Co., 8mi E Centralia, 7.IX.1993, J. D. Pinto ( 1 female, 1 male, USNMENT00977422-00977423 (UCRC)). IL, Piatt Co., White Heath, 4.VI.1939, J. C. Dirks (1 female, USNMENT00977327 (USNM)). IN, Orange Co., Mineral Springs, IX-1925 (1 female, USNMENT00977367 (USNM)). IN, Tippecanoe Co., Lafayette, no date, Satterthwaite (1 female, USNMENT00993566 (USNM)). KS, Douglas Co., Breidenthal Biological Reserve, 21.VII.1979, R. J. McGinley (1 female, USNMENT00977358 (USNM)). KS, Geary Co., watershed 20B, 2005-062, Konza Prairie Biological Station, 30.VII9.VIII.2005, Malaise trap, Zolnerowich \& Kula (2 females, 2 males, USNMENT00993612-00993615 (USNM)). KS, Geary Co., watershed 20B, 2006003, Konza Prairie Biological Station, 18.V-22.V.2006, Malaise trap, Zolnerowich \& Metlevski (4 females, 1 male, USNMENT00993638-00993642 (USNM)). KS, Geary Co., watershed 20C, 2005003, Konza Prairie Biological Station, 5. V-12.V.2005, Malaise trap, Zolnerowich \& Kula (3 females, 1 male, USNMENT00993057-00993059, 00993061 (USNM)). KS, Geary Co., watershed 20C, 2005-018, Konza Prairie Biological 
Station, 22.V-27.V.2005, Malaise trap, Zolnerowich \& Kula (13 females, 57 males, USNMENT00993065-00993075, 00993077-00993080, 00993091-00993120, 00993376-00993380, 00993382-00993397, 00993548, 00993550-00993552 (USNM)). KS, Geary Co., watershed 20C, 2005023, Konza Prairie Biological Station, 27.V-6.VI.2005, Malaise trap, Zolnerowich \& Kula (30 females, 99 males, USNMENT00993014-00993021, 0099302300993049, 00993051-00993052, 00993064, 00993121-00993124, 00993174-00993196, 00993198-00993199, 00993201-00993210, 00993212-00993228, 00993255-00993269, 00993398-00993406, 00993408-00993418 (USNM)). KS, Geary Co., watershed 20C, 2005-028, Konza Prairie Biological Station, 6.VI-13.VI.2005, Malaise trap, Zolnerowich \& Kula (21 females, 32 males, USNMENT00993444-00993453, 00993455-00993456, 00993458-00993460, 00993462-00993476, 00993478-00993495, 00993497, 00993650-00993653 (USNM)). KS, Geary Co., watershed 20C, 2005-033, Konza Prairie Biological Station, 13.VI-20. VI.2005, Malaise trap, Zolnerowich \& Kula (22 females, 29 males, 2 unknowns, USNMENT00993162-00993168, 00993170-00993171, 00993420-00993426, 00993428-00993443, 00993530-00993547, 00993658-00993660 (USNM)). KS, Geary Co., watershed 20C, 2005-038, Konza Prairie Biological Station, 20.VI-27. VI.2005, Malaise trap, Zolnerowich \& Kula (18 females, 31 males, USNMENT00993157-00993161, 0099327000993288, 00993290-00993312, 0099364300993644 (USNM)). KS, Geary Co., watershed 20C, 2005-043, Konza Prairie Biological Station, 27.VI-6.VII.2005, Malaise trap, Zolnerowich \& Kula (4 females, 3 males, USNMENT00993606-00993611, 00993655 (USNM)). KS, Geary Co., watershed 20C, 2005-048, Konza Prairie Biological Station, 6.VII-11.VII.2005, Malaise trap, Zolnerowich \& Kula (9 females, 33 males, USNMENT00993000-00993002, 00993004-00993012, 00993053-00993054, 00993056, 00993062-00993063, 0099322900993248, 00993250-00993254 (USNM)). KS, Geary Co., watershed 20C, 2005-053, Konza Prairie Biological Station, 11.VII-20. VII.2005, Malaise trap, Zolnerowich \& Kula (4 males, USNMENTO099361900993622 (USNM)). KS, Geary Co., watershed 20C, 2005-058, Konza Prairie Biological Station, 20.VII-30.VII.2005, Malaise trap, Zolnerowich \& Kula (1 female, 3 males, USNMENT00993645-00993648 (USNM)). KS, Geary Co., watershed 20C, 2005-063, Konza Prairie Biological Station, 30.VII-9.VIII.2005, Malaise trap, Zolnerowich \& Kula (11 females, 6 males, USNMENT00993616-00993618, 00993654, 00993966-00993978 (USNM)). KS, Geary Co., watershed 20C, 2005-068, Konza Prairie Biological Station, 9.VIII-16.VIII.2005, Malaise trap, Zolnerowich \& Kula (5 females, 6 males, USNMENTO099336400993368, 00993370-00993375 (USNM)). KS, Geary Co., watershed 20C, 2005-073, Konza Prairie Biological Station, 16.VIII-26. VIII.2005, Malaise trap, Zolnerowich \& Kula (10 females, 19 males, 2 unknowns, USNMENT00993125-00993126, 00993128-00993129, 00993131-00993133, 00993135-00993148, 00993150, 0099315200993154, 00993156, 00993629-00993633 (USNM)). KS, Geary Co., watershed 20C, 2006-004, Konza Prairie Biological Station, 18.V-22.V.2006, Malaise trap, Zolnerowich \& Metlevski (2 females, 1 male, USNMENT00993635-00993637 (USNM)). KS, Geary Co., watershed 20C, 2006-012, Konza Prairie Biological Station, 29.V-5.VI.2006, Malaise trap, Zolnerowich \& Metlevski (1 female, 3 males, USNMENT00993603-00993605, 00993656 (USNM)). KS, Geary Co., watershed 20C, 2006-024, Konza Prairie Biological Station, 26.VI-3.VII.2006, Malaise trap, Zolnerowich \& Metlevski (1 male, USNMENT00993657 (USNM)). 
KS, Reno Co., S of Medora, $38^{\circ} 07^{\prime} 37^{\prime \prime} \mathrm{N}$ 97 $50^{\prime} 47^{\prime \prime} \mathrm{W}$, Sand Hills State Park, 22. V.2009, G. F. Hevel \& J. F. Hevel (1 male, USNMENT00993513 (USNM)). LA, Madison Parish, Airpl. >20, Tallulah, no date (1 female, USNMENT00977016 (USNM)). LA, Saint Landry Parish, Opelousas, no date, G. R. Pilate (2 females, USNMENT00977313, 00977364 (USNM)). MA, Middlesex Co., Reading, 30.IX.1909, Moss (1 female, USNMENT00977334 (USNM)). MD, Anne Arundel Co., "The Dessert", Sand Barrens, Glendening Preserve, $38^{\circ} 48^{\prime} 24.08^{\prime \prime} \mathrm{N}$ $76^{\circ} 42^{\prime} 5.85^{\prime \prime} \mathrm{W}$, Jug Bay Wetlands Sanctuary, 9.V-29.V.2007, Gates et al. (1 unknown, USNMENT00993624 (USNM)). MD, Anne Arundel Co., "The Dessert", Sand Barrens, Glendening Preserve, $38^{\circ} 48^{\prime} 24.08^{\prime \prime} \mathrm{N} 76^{\circ} 42^{\prime} 5.85^{\prime \prime} \mathrm{W}$, Jug Bay Wetlands Sanctuary, 9.VII-25.VII.2007, Malaise trap, Gates, Buffington \& Kula (1 male, USNMENT00993965 (USNM)). MD, Calvert Co., Huntingtown, Kings Landing Road, 1.VI.1998, reared (1 female, 3 males, USNMENT0097714300977146 (USNM)). MD, Calvert Co., Warrior's Rest Sanctuary, "Beach 1", $38^{\circ} 32.154^{\prime} \mathrm{N} 76^{\circ} 31.071^{\prime} \mathrm{W}$, American Chestnut Land Trust, 31.VIII-18. IX.2009, canopy trap, M. Gates et al. (1 male, USNMENT00993960 (USNM)). MD, Calvert Co., Warrior's Rest Sanctuary, "Beach trap", $38^{\circ} 32.154^{\prime} \mathrm{N}$ $76^{\circ} 31.071^{\prime} \mathrm{W}$, American Chestnut Land Trust, 1.X.2006, SEL Hym Unit (1 female, USNMENT00993348 (USNM)). MD, Calvert Co., Warrior's Rest Sanctuary, "Fern Gully CT", $38^{\circ} 32^{\prime} 00.11^{\prime \prime} \mathrm{N}$ $76^{\circ} 32^{\prime} 30.60^{\prime \prime} \mathrm{W}$, American Chestnut Land Trust, 7.VI-22.VI.2007, Malaise trap, SEL Hym Unit (1 unknown, USNMENT00993089 (USNM)). MD, Calvert Co., Warrior's Rest Sanctuary, "Monster $\quad \mathrm{MT}^{\prime \prime}, \quad 38^{\circ} 31^{\prime} 54.04^{\prime \prime} \mathrm{N}$ $76^{\circ} 32^{\prime} 31.62^{\prime \prime} \mathrm{W}$, American Chestnut Land Trust, 8.VI-22.VI.2007, Malaise trap, M. Gates et al. (1 unknown, USNMENT00993625 (USNM)). MD, Calvert Co., Warrior's Rest Sanctuary, "North Source", 38³1'58.97"N 76³2'30.99"W, American Chestnut Land Trust, 22. VI.2007, Malaise trap, SEL Hym Unit (1 female, USNMENT00993346 (USNM)). MD, Calvert Co., Warrior's Rest Sanctuary, "Oak", $\quad 38^{\circ} 32.006^{\prime} \mathrm{N} \quad 76^{\circ} 32.646^{\prime} \mathrm{W}$, American Chestnut Land Trust, 12.IV25.IV.2008, Malaise trap, SEL Hym Unit (1 male, USNMENT00993090 (USNM)). MD, Calvert Co., Warrior's Rest Sanctuary, "Oak", 38³2.006' N 76³2.646' W, American Chestnut Land Trust, 27.V-8. VI.2008, Malaise trap, SEL Hym Unit (1 male, USNMENT00993964 (USNM)). MD, Calvert Co., Warrior's Rest Sanctuary, "Phragmites CT", 38 32'11.47"N $76^{\circ} 31^{\prime} 12.58^{\prime \prime} \mathrm{W}$, American Chestnut Land Trust, 9.VII-25.VII.2007, Malaise trap, SEL Hym Unit (1 unknown, USNMENT00993558 (USNM)). MD, Calvert Co., Warrior's Rest Sanctuary, "Spartina cyanosuroides", 38³2.198'N $76^{\circ} 31.18^{\prime} \mathrm{W}$, American Chestnut Land Trust, 9.VI-23.VI.2008, canopy trap, M. Gates et al. (1 female, USNMENT00993962 (USNM)). MD, Calvert Co., Warrior's Rest Sanctuary, "Thin Branch", $38^{\circ} 32^{\prime} 00.02^{\prime \prime} \mathrm{N} 76^{\circ} 32^{\prime} 30.72^{\prime \prime} \mathrm{W}$, American Chestnut Land Trust, 22.VII.2007, canopy trap, M. Gates et al. (1 male, USNMENT00993961 (USNM)). MD, Calvert Co., Warrior's Rest Sanctuary, $38.53153^{\circ} \mathrm{N} 76.528591^{\circ} \mathrm{W}$, American Chestnut Land Trust, 1.VII-15.VII.2006, Malaise trap, SEL Hym Unit (1 female, 4 males, USNMENT00993351-00993355 (USNM)). MD, Calvert Co., Warrior's Rest Sanctuary, $38.53153^{\circ} \mathrm{N} 76.528591^{\circ} \mathrm{W}$, American Chestnut Land Trust, 15.VII28.VII.2006, Malaise trap, SEL Hym Unit (1 female, USNMENT00993350 (USNM)). MD, Calvert Co., Warrior's Rest Sanctuary, $38.5360641^{\circ} \mathrm{N} 76.5175095^{\circ} \mathrm{W}$, American Chestnut Land Trust, 2.VI-17. 
VI.2006, Malaise trap, M. Gates (1 female, 1 male, USNMENT0099368000993681 (USNM)). MD, Calvert Co., Warrior's Rest Sanctuary, 38 $31^{\prime} 56.37^{\prime \prime} \mathrm{N}$ 76 32'35.71"W, American Chestnut Land Trust, 1.IX-14.IX.2007, Malaise trap, SEL Hym Unit (1 female, USNMENT00993360 (USNM)). MD, Calvert Co., Warrior's Rest Sanctuary, $38^{\circ} 31^{\prime} 56.37^{\prime \prime} \mathrm{N} 76^{\circ} 32^{\prime} 35.71^{\prime \prime} \mathrm{W}$, American Chestnut Land Trust, 9.VIII-28. VIII.2007, Malaise trap, SEL Hym Unit (1 female, USNMENT00993347 (USNM)). MD, Calvert Co., Warrior's Rest Sanctuary, $38^{\circ} 32^{\prime} 00.11^{\prime \prime} \mathrm{N} \quad 76^{\circ} 32^{\prime} 30.60^{\prime \prime} \mathrm{W}$, American Chestnut Land Trust, 1.VII15.VII.2006, Malaise trap, SEL Hym Unit (1 female, 4 males, USNMENT00993084-00993088 (USNM)). MD, Calvert Co., Warrior's Rest Sanctuary, beach strand, $38^{\circ} 32^{\prime} 00.11^{\prime \prime} \mathrm{N}$ 76³2'30.60"W, American Chestnut Land Trust, 28.VIII.2007, sweeping, M. Gates et al. (1 female, 1 unknown, USNMENT00993626-00993627 (USNM)). MD, Calvert Co., Warrior's Rest Sanctuary, beach, $38^{\circ} 31^{\prime} 56.37^{\prime \prime} \mathrm{N} \quad 76^{\circ} 32^{\prime} 35.71^{\prime \prime} \mathrm{W}$, American Chestnut Land Trust, V-2006, Malaise trap, M. Gates et al. (1 female, USNMENT00993628 (USNM)). MD, Calvert Co., Warrior's Rest Sanctuary, by Parkers Creek, American Chestnut Land Trust, 25.VIII-8.IX.2006, Malaise trap, Gates \& Kula (11 females, 8 males, 3 unknowns, USNMENT0097710200977122, 00993319 (USNM)). MD, Calvert Co., Warrior's Rest Sanctuary, by Parkers Creek, American Chestnut Land Trust, 28.VII-12.VIII.2006, Malaise trap, Gates \& Kula (1 female, 1 male, 1 unknown, USNMENT00977123-00977125 (USNM)). MD, Calvert Co., hardwood forest, 7km S Prince Frederick, VII-1987 - 10-IX-1987, BRC Hym Team (1 female, 1 male, USNMENT00977426, 00977504 (UCRC)). MD, Montgomery Co., Cabin John, 3.VI.1917, R. M. Fouts
(1 female, USNMENT00977365 (USNM)). MD, Montgomery Co., Cabin John, 30. VI.1917, R. M. Fouts (1 male, USNMENT00977331 (USNM)). MD, Montgomery Co., Glen Echo, 26.VIII.1922, R. M. Fouts (1 female, USNMENT00977366 (USNM)). MD, Montgomery Co., Plummers Island, 24.II-7. V.2006, Malaise trap, D. Smith \& J. Brown (2 females, USNMENT0099389800993899 (USNM)). MD, Prince George's Co., Patuxent Research Refuge, 14. VIII-30.VIII.2006, Malaise trap, S. J. Scheffer (1 female, USNMENT00993679 (USNM)). MD, Prince George's Co., Patuxent Research Refuge, 15.V-22.V.2006, Malaise trap, S. J. Scheffer (3 unknowns, USNMENT00993527-00993529 (USNM)). MD, Prince George's Co., Patuxent Research Refuge, 22.V-30.V.2006, Malaise trap, S. J. Scheffer (3 males, USNMENT00993676-00993678 (USNM)). MN, Anoka Co., 97-59, Cedar Creek Natural History Area, 20.VIII.1997, S. J. Scheffer (1 male, USNMENT00977147 (USNM)). MN, Anoka Co., 97-59, Cedar Creek Natural History Area, 20. VIII.1997, reared, S. J. Scheffer (1 female, 2 males, USNMENT0097730400977306 (USNM)). MO, Boone Co., Columbia, 13.V.1970, F. D. Parker (1 female, USNMENT00977350 (USNM)). MO, Boone Co., Columbia, 16.IV-30. IV.1968, Malaise trap, F. D. Parker (2 males, USNMENT00977353, 00993560 (USNM)). MO, Boone Co., Columbia, 2. VIII.1970, F. D. Parker (1 female, 1 male, USNMENT00977311, 00977351 (USNM)). MO, Boone Co., Columbia, 4. IX.1968, Malaise trap, F. D. Parker (2 females, USNMENT00977308, 00977352 (USNM)). MO, Boone Co., Columbia, 9. XII.1967, Malaise trap, F. D. Parker (1 female, USNMENT00977310 (USNM)). MO, Greene Co., Springfield, 19.VIII.2003, J. D. Pinto (1 male, 
USNMENT00977428 (UCRC)). NC, Granville Co., $36.165^{\circ} \mathrm{N} 78.739^{\circ} \mathrm{W}$, Picture Creek area, 19.VIII-2.IX.2009, Malaise trap, R. L. Blinn \& I. Miko (1 female, USNMENT00993173 (USNM)). NC, New Hanover Co., 97-11, Carolina Beach State Park, 21.II.1997, reared, S. Scheffer (13 females, 1 male, USNMENT00993809-00993822 (USNM)). NC, New Hanover Co., 97-12, Carolina Beach State Park, 21.II.1997, reared, S. Scheffer (6 females, 6 males, USNMENT00993796-00993807 (USNM)). NC, New Hanover Co., Flytrap Trail, 9711, Carolina Beach State Park, 21. II.1997, reared, S. Scheffer (2 females, USNMENT00977493-00977494 (UCRC)). NC, New Hanover Co., Holly Hill, 9839, Carolina Beach State Park, 26. I.1998, reared, S. Scheffer (3 females, 2 males, USNMENT00993791-00993795 (USNM)). NC, Scotland Co., $34.980^{\circ} \mathrm{N}$ $79.558^{\circ} \mathrm{W}$, Sandhills Game Land, 19.IV4.V.2010, Malaise trap, R. L. Blinn (1 male, USNMENT00993514 (USNM)). NE, no date (1 female, USNMENT00977359 (USNM)). NV, Clark Co., Mesquite, 16.VII.2004, Malaise trap, W. Wiesenborn (1 female, USNMENT01081075 (USNM)). NY, Orange Co., Durlandville, 1917, S. W. Frost (1 female, USNMENT00977329 (USNM)). NY, Orange Co., Pine Island, 6. VIII.1917, S. W. Frost (1 female, USNMENT00977330 (USNM)). NY, Suffolk Co., Long Island, Islip, 25.IX.1934, F. S. Blanton (1 female, USNMENT00977314 (USNM)). OK, Latimer Co., environs of Red Oak, VI-1994, flight intercept trap, K. Stephan (1 female, USNMENT00977424 (UCRC)). OK, Oklahoma Co., Remington Park, Oklahoma City, 5.VIII.1999, sweeping, M. L. Buffington (2 females, USNMENT0097742000977421 (TAMU)). PA, Adams Co., Arendtsville, 10.IX.1921, S. W. Frost (1 female, USNMENT00653494 (USNM)).
PA, Adams Co., Arendtsville, 10. VII.1921, S. W. Frost (1 male, USNMENT00653508 (USNM)). PA, Adams Co., Arendtsville, 1919 (2 females, USNMENT00977355-00977356 (USNM)). PA, Adams Co., Arendtsville, VIII1920, S. W. Frost (1 male, USNMENT00653488 (USNM)). PA, Adams Co., Arendtsville, no date, S. W. Frost (12 females, 3 males, 1 unknown, USNMENT00653492-00653493, 00653498, 00977336-00977348 (USNM)). SC, Berkeley Co., N. Honey Hill Road, 97-7, Francis Marion National Forest, 19. II.1997, S. Scheffer (1 female, 4 males, USNMENT00977256-00977260 (USNM)). SC, Berkeley Co., N. Honey Hill Road, 97-7, Francis Marion National Forest, 19.II.1997, reared, S. Scheffer (1 female, 1 male, USNMENT00977214, 00993808 (USNM)). SC, Berkeley Co., N. Honey Hill Road, 97-7, Francis Marion National Forest, 19.II.1997, reared, S. J. Scheffer (3 females, USNMENT00977300-00977302 (USNM)). SC, Berkeley Co., N. Honey Hill Road, Francis Marion National Forest, 19. II.1997, reared, S. Scheffer (20 females, 21 males, USNMENT0097721500977255 (USNM)). TN, Blount Co., Cades Cove, MT3 / ATBI plot, $35^{\circ} 35.31^{\prime} \mathrm{N} 83^{\circ} 50.17^{\prime} \mathrm{W}$, Great Smoky Mountains National Park, 8.V-21. V.2001, Malaise trap, R. Hightower \& J. Burbank (1 male, USNMENT00993979 (USNM)). TN, Cocke Co., Hartford, 24. VI.1986, J. D. Pinto (1 female, USNMENT00977527 (UCRC)). TX, Bandera Co., Lost Maples State Natural Area, 17.IV.1993, G. Zolnerowich (1 female, USNMENT00993359 (USNM)). TX, Bandera Co., Lost Maples State Natural Area, 19.VIII.1988, J. Heraty (1 female, USNMENT00977498 (UCRC)). TX, Brazos Co., College Station, 20.VI24.VI.1986, Malaise trap, R. Wharton (1 male, USNMENT00993869 (USNM)). 
TX, Brazos Co., Lick Creek Park, College Station, 2.IV-7.IV.2005, Malaise trap, K. Zhaurova (2 females, USNMENT00993356-00993357 (USNM)). TX, Brazos Co., Lick Creek Park, College Station, 20.IX.1992, G. Zolnerowich (1 male, USNMENT00993665 (USNM)). TX, Brazos Co., Lick Creek Park, College Station, 7.V.2005, R. A. Wharton (1 male, USNMENT00993358 (USNM)). TX, Brazos Co., Lick Creek Park, College Station, 7.V.2005, K. Zhaurova \& R. A. Wharton (1 female, USNMENT00993563 (USNM)). TX, Brazos Co., Lick Creek Park, College Station, 8.X-22.X.2001, Malaise trap, R. Wharton (1 female, USNMENT00993082 (USNM)). TX, Brazos Co., Lick Creek Park, College Station, summer 1987, yellow pan trap, J. Heraty \& J. Woolley (2 males, USNMENT00977496-00977497 (UCRC)). TX, Brazos Co., Lick Creek Park, front field, 1991/042, College Station, 11.VII.1991, J. B. Woolley (1 female, USNMENT00993663 (USNM)). TX, Brazos Co., Lick Creek Park, meadow edge, College Station, 9.IV-17. IV.1987, Malaise trap, J. M. Heraty (1 female, USNMENT00977425 (UCRC)). TX, Dallas Co., Dallas, 1.V.1909, W. D. Pierce (1 male, USNMENT00993686 (USNM)). TX, Hidalgo Co., 26 $09^{\prime} 33.01^{\prime \prime} \mathrm{N}$ 9757'32.67"W, Weslaco, 12.XI.2007, reared, Hernandez (3 females, USNMENT00993787-00993789 (USNM)). TX, Hidalgo Co., 26 $09^{\prime} 33.01^{\prime \prime} \mathrm{N}$ 9757'32.67"W, Weslaco, 28.XI.2007, reared, Hernandez (1 female, USNMENT00993786 (USNM)). TX, Hidalgo Co., Bentsen Rio Grande Valley State Park, 30.XI-2.XII.1978, E. E. Grissell \& A. S. Menke (1 male, USNMENT00653495 (USNM)). TX, Hidalgo Co., Weslaco, 20.IX.2007, reared, Hernandez (1 female, USNMENT00993790 (USNM)). TX, Jeff Davis Co., 16mi N Fort Davis, Little Aguja Canyon, Ranch to Market Road 1832, 6.IX.1984, T. O. Robbins (1 male, USNMENT00977333 (USNM)). TX, Jim Wells Co., La Copita Ranch, 8mi NW Ben Bolt, 28.IX-30. IX.1990, R. Wharton (1 male, USNMENT00977499 (UCRC)). TX, Lamar Co., lot 6, Camp Maxey, III-2003 - VII2003, W. Godwin (2 males, USNMENT00993667-00993668 (USNM)). TX, Robertson Co., 8mi E Hearne, 21. IV-27.IV.1991, M. Hallmark (1 female, 1 male, USNMENT00993081, 00993083 (USNM)). TX, Robertson Co., 8mi E Hearne, VI-1991, M. Hallmark (2 females, 1 male, USNMENT0099387300993875 (USNM)). TX, San Patricio Co., Welder Wildlife Foundation Park, 1. XI.1988, J. Heraty (1 male, USNMENT00977495 (UCRC)). TX, Terrell Co., $\sim 5 \mathrm{mi}$ W Sanderson, 18.VII.1997, M. Gates (1 male, USNMENT00977500 (UCRC)). TX, Travis Co., Longhollow LCRA, Austin, 10.IV-23.IV.1993, Malaise trap, R. Wharton (1 female, USNMENT00977427 (UCRC)). TX, Uvalde Co., Speir Ranch, 3mi NW Uvalde, 3. V.1977, Malaise trap, T. Eichlin \& M. Wasbauer (1 male, USNMENT01081000 (USNM)). VA, Arlington Co., East Falls Church, 25-VI (1 female, USNMENT00977368 (USNM)). VA, Arlington Co., East Falls Church, 30.VIII.1934 (1 male, USNMENT00977332 (USNM)). VA, Arlington Co., Rosslyn, 16.VI.1920 (1 male, USNMENT00977307 (USNM)). VA, Essex Co., $37^{\circ} 52^{\prime} \mathrm{N} 76^{\circ} 48^{\prime} \mathrm{W}, 1 \mathrm{mi}$ SE Dunnsville, 3.VIII-20.VIII.1996, Malaise trap, D. R. Smith (1 female, USNMENT00993562 (USNM)). VA, Fairfax Co., $38^{\circ} 50^{\prime} \mathrm{N} 77^{\circ} 12^{\prime} \mathrm{W}$, nr. Annandale, 28.V-3.VI.2006, Malaise trap, D. R. Smith (1 female, USNMENT00993316 (USNM)). VA, Fairfax Co., $38^{\circ} 50^{\prime} \mathrm{N} 77^{\circ} 12^{\prime} \mathrm{W}$, nr. Annandale, 9.VII-15.VII.2006, Malaise trap, D. R. Smith (1 female, USNMENT00977140 (USNM)). VA, Fairfax Co., $38^{\circ} 57.9^{\prime} \mathrm{N}$ 
$77^{\circ} 09.4^{\prime} \mathrm{W}$, Turkey Run Park, 20.VI-2. VII.2008, Malaise trap, D. R. Smith (1 female, USNMENT00993752 (USNM)). VA, Fairfax Co., $38^{\circ} 57.9^{\prime} \mathrm{N} 77^{\circ} 09.4^{\prime} \mathrm{W}$, Turkey Run Park, 22.VI-6.VII.2006, Malaise trap, D. Smith (1 female, USNMENT00993507 (USNM)). VA, Fairfax Co., $38^{\circ} 57.9^{\prime} \mathrm{N} \quad 77^{\circ} 09.4^{\prime} \mathrm{W}$, Turkey Run Park, 7.VI-21.VI.2006, Malaise trap, D. Smith (1 female, USNMENT00993508 (USNM)). VA, Fairfax Co., $38^{\circ} 58^{\prime} \mathrm{N} 77^{\circ} 09.6^{\prime} \mathrm{W}$, Turkey Run West, 18.V-30.V.2007, Malaise trap, D. Smith (1 female, USNMENT00977138 (USNM)). VA, Fairfax Co., $38^{\circ} 58^{\prime} \mathrm{N}$ $77^{\circ} 09.6^{\prime} \mathrm{W}$, Turkey Run West, 19.IX-11. X.2006, Malaise trap, D. Smith (1 female, 1 male, USNMENT00977139, 00993363 (USNM)). VA, Fairfax Co., $38^{\circ} 58^{\prime} \mathrm{N} \quad 77^{\circ} 09.6^{\prime} \mathrm{W}$, Turkey Run West, 31.V-13.VI.2007, Malaise trap, D. Smith (2 females, 3 males, USNMENT00977132-00977136 (USNM)). VA, Fairfax Co., $38^{\circ} 58^{\prime} \mathrm{N} 77^{\circ} 09.6^{\prime} \mathrm{W}$, Turkey Run West, 5.VIII-24.VIII.2007, Malaise trap, D. Smith (1 male, USNMENT00977137 (USNM)). VA, Fairfax Co., $38^{\circ} 59.4^{\prime} \mathrm{N} 77^{\circ} 15.2^{\prime} \mathrm{W}$, Great Falls Park, 1.VII-15.VII.2009, Malaise trap, D. R. Smith (1 unknown, USNMENT00993557 (USNM)). VA, Fairfax Co., $38^{\circ} 59.4^{\prime} \mathrm{N} 77^{\circ} 15.2^{\prime} \mathrm{W}$, Great Falls Park, 18.VII-4.VIII.2008, Malaise trap, D. R. Smith (1 female, 1 male, USNMENT00993876-00993877 (USNM)). VA, Fairfax Co., $38^{\circ} 59.4^{\prime} \mathrm{N} 77^{\circ} 15.2^{\prime} \mathrm{W}$, Great Falls Park, 18.VII-4.VIII.2008, D. R. Smith (1 female, USNMENT00993878 (USNM)). VA, Fairfax Co., $38^{\circ} 59.4^{\prime} \mathrm{N}$ $77^{\circ} 15.2^{\prime} \mathrm{W}$, Great Falls Park, 3.VII-17. VII.2008, D. R. Smith (1 male, USNMENT00993754 (USNM)). VA, Fairfax Co., $38^{\circ} 59.4^{\prime} \mathrm{N} 77^{\circ} 15.2^{\prime} \mathrm{W}$, Great Falls Park, 31.VII-17.VIII.2009, Malaise trap, D. R. Smith (1 female, USNMENT00993753 (USNM)). VA, Fairfax Co., MT E-1, Dyke Marsh Wildlife
Preserve, 1.VIII.1999, E. M. Barrows (1 female, 2 males, USNMENT0099398000993982 (USNM)). VA, Fairfax Co., MT E-2, Dyke Marsh Wildlife Preserve, 10.V-17.V.1998, E. M. Barrows (1 male, USNMENT00993983 (USNM)). VA, Fairfax Co., MT E-2, Dyke Marsh Wildlife Preserve, 6.XII.1998, E. M. Barrows (1 female, 1 male, USNMENT00993985-00993986 (USNM)). VA, Fairfax Co., MT F-2, Dyke Marsh Wildlife Preserve, 20.VI.1999, E. M. Barrows (1 female, USNMENT00993984 (USNM)). VA, Fairfax Co., trap \#2, $38^{\circ} 59.4^{\prime} \mathrm{N} 77^{\circ} 15.26^{\prime} \mathrm{W}$, Great Falls, 23. VIII-18.IX.2006, Malaise trap, D. Smith (3 females, 1 male, USNMENT0097714100977142, 00993555-00993556 (USNM)). VA, Fairfax Co., $\sim 0.25 \mathrm{mi}$ NE jct. Gallows Road \& I-495, 38 $50^{\prime} \mathrm{N} 77^{\circ} 12^{\prime} \mathrm{W}$, Holmes Run, 10.VII-16.VII.2011, Malaise trap, D. Smith (1 female, 1 male, USNMENT00993509-00993510 (USNM)). VA, Fairfax Co., $\sim 0.25 \mathrm{mi}$ NE jct. Gallows Road \& I-495, $38^{\circ} 50^{\prime} \mathrm{N} 77^{\circ} 12^{\prime} \mathrm{W}$, Holmes Run, 17.VII-23.VII.2011, Malaise trap, D. Smith (2 females, USNMENT00993511-00993512 (USNM)). VA, Fairfax Co., $\sim 0.25 \mathrm{mi}$ NE jct. Gallows Road \& I-495, 38 $50^{\prime} \mathrm{N} 77^{\circ} 12^{\prime} \mathrm{W}$, Holmes Run, 20.VII-26.VII.2008, Malaise trap, D. R. Smith (1 female, USNMENT00993522 (USNM)). VA, Fairfax Co., $\sim 0.25 \mathrm{mi}$ NE jct. Gallows Road \& I495, $38^{\circ} 50^{\prime} \mathrm{N} 77^{\circ} 12^{\prime} \mathrm{W}$, Holmes Run, 22.V-28.V.2011, Malaise trap, D. Smith (1 male, USNMENT00993521 (USNM)). VA, Louisa Co., 4mi S Cuckoo, 3.VIII.1985, Malaise trap, J. Kloke \& D. R. Smith (1 female, USNMENT00977326 (USNM)). VA, Prince William Co., Conservancy Campground area, $38^{\circ} 49.484^{\prime} \mathrm{N}$ $77^{\circ} 41.362^{\prime} \mathrm{W}$, Bull Run Mountains, 11. VI-24.VI.2011, Malaise trap, D. Smith (1 male, USNMENT00993505 (USNM)). VA, Prince William Co., Conservancy Campground area, $38^{\circ} 49.484^{\prime} \mathrm{N}$ 
$77^{\circ} 41.362^{\prime} \mathrm{W}$, Bull Run Mountains, 13. V-27.V.2011, Malaise trap, D. Smith (1 female, USNMENT00993506 (USNM)). VA, Prince William Co., Conservancy Campground area, $38^{\circ} 49.484^{\prime} \mathrm{N}$ $77^{\circ} 41.362^{\prime} \mathrm{W}$, Bull Run Mountains, 21. IX-18.X.2012, Malaise trap, D. Smith (2 females, USNMENT00993317-00993318 (USNM)). VA, Prince William Co., Conservancy Campground area, $38^{\circ} 49.484^{\prime} \mathrm{N}$ $77^{\circ} 41.362^{\prime} \mathrm{W}$, Bull Run Mountains, 24. IX-4.XI.2013, Malaise trap, D. Smith (1 male, USNMENT00993321 (USNM)). VA, Prince William Co., Conservancy Campground area, $38^{\circ} 49.484^{\prime} \mathrm{N} 77^{\circ} 41.362^{\prime} \mathrm{W}$, Bull Run Mountains, 24.VIII-20. IX.2012, Malaise trap, D. Smith (2 females, 1 male, USNMENT0099351800993520 (USNM)). VA, Prince William Co., Conservancy Campground area, $38^{\circ} 49.484^{\prime} \mathrm{N} 77^{\circ} 41.362^{\prime} \mathrm{W}$, Bull Run Mountains, 25.VI-7.VII.2011, Malaise trap, D. Smith (1 female, USNMENT00993554 (USNM)). VA, Prince William Co., Conservancy Campground area, $38^{\circ} 49.484^{\prime} \mathrm{N} 77^{\circ} 41.362^{\prime} \mathrm{W}$, Bull Run Mountains, 28.V-10.VI.2011, Malaise trap, D. Smith (1 male, USNMENT00993515 (USNM)). VA, Prince William Co., Conservancy Campground area, $38^{\circ} 49.484^{\prime} \mathrm{N} 77^{\circ} 41.362^{\prime} \mathrm{W}$, Bull Run Mountains, 29.VIII-23.IX.2013, Malaise trap, D. Smith (2 males, USNMENT00993503-00993504 (USNM)). VA, Prince William Co., Jackson Hollow Recreation Area, stream, 38 $52.645^{\prime} \mathrm{N}$ $77^{\circ} 41.374^{\prime} \mathrm{W}$, Bull Run Mountains, 1. IX-30.IX.2011, Malaise trap, D. Smith (1 female, USNMENTO0993498 (USNM)). VA, Prince William Co., Jackson Hollow Recreation Area, stream, 38 $52.645^{\prime} \mathrm{N}$ $77^{\circ} 41.374^{\prime} \mathrm{W}$, Bull Run Mountains, 10.VIII31.VIII.2011, Malaise trap, D. Smith (4 females, USNMENT00993499-00993502 (USNM)). VA, Prince William Co., Jackson Hollow Recreation Area, stream, $38^{\circ} 52.645^{\prime} \mathrm{N} 77^{\circ} 41.374^{\prime} \mathrm{W}$, Bull
Run Mountains, 22.VII-9.VIII.2011, Malaise trap, D. Smith (1 male, USNMENT00993517 (USNM)). VA, Prince William Co., Jackson Hollow Recreation Area, stream, $38^{\circ} 52.645^{\prime} \mathrm{N} 77^{\circ} 41.374^{\prime} \mathrm{W}$, Bull Run Mountains, 28.V-10.VI.2011, Malaise trap, D. Smith (1 female, USNMENT00993516 (USNM)). WV, Hardy Co., 2200ft, $38^{\circ} 55^{\prime} \mathrm{N} 78^{\circ} 49^{\prime} \mathrm{W}$, $3 \mathrm{mi}$ NE Mathias, 1.VIII-18.VIII.2008, Malaise trap, D. R. Smith (2 males, USNMENT00993672-00993673 (USNM)). WV, Hardy Co., 2200ft, $38^{\circ} 55^{\prime} \mathrm{N}$ $78^{\circ} 49^{\prime} \mathrm{W}, 3 \mathrm{mi}$ NE Mathias, 17.VI-30. VI.2008, Malaise trap, D. R. Smith (10 females, 2 males, USNMENT0099333600993345, 00993525-00993526 (USNM)). WV, Hardy Co., 2200ft, $38^{\circ} 55^{\prime} \mathrm{N}$ $78^{\circ} 49^{\prime} \mathrm{W}, 3 \mathrm{mi}$ NE Mathias, 19.VIII-8. IX.2008, Malaise trap, D. R. Smith (1 female, 1 male, USNMENT0099352300993524 (USNM)). WV, Hardy Co., $2200 \mathrm{ft}, 38^{\circ} 55^{\prime} \mathrm{N} 78^{\circ} 49^{\prime} \mathrm{W}, 3 \mathrm{mi} \mathrm{NE} \mathrm{Ma}-$ thias, 30.V-17.VI.2008, Malaise trap, D. R. Smith (9 females, 5 males, USNMENT00993322-00993335 (USNM)). WV, Hardy Co., $38^{\circ} 55^{\prime} \mathrm{N} 78^{\circ} 49^{\prime} \mathrm{W}, 3 \mathrm{mi}$ NE Mathias, 1.VII-16.VII.2008, Malaise trap, D. R. Smith (8 females, 3 males, USNMENT00993887-00993897 (USNM)). WV, Hardy Co., $38^{\circ} 55^{\prime} \mathrm{N} 78^{\circ} 49^{\prime} \mathrm{W}, 3 \mathrm{mi}$ NE Mathias, 17.VI-30.VI.2008, Malaise trap, D. R. Smith (3 females, USNMENT00993882-00993884 (USNM)). WV, Hardy Co., $38^{\circ} 55^{\prime} \mathrm{N} 78^{\circ} 49^{\prime} \mathrm{W}, 3 \mathrm{mi}$ NE Mathias, 17.VII-31.VII.2008, Malaise trap, D. R. Smith (3 females, 2 males, USNMENT00993674-00993675, 00993879-00993881 (USNM)). WV, Hardy Co., $38^{\circ} 55^{\prime} \mathrm{N} 78^{\circ} 49^{\prime} \mathrm{W}$, 3mi NE Mathias, 23.VI-10.VII.2006, D. R. Smith (8 females, USNMENT00993900-00993907 (USNM)). WV, Hardy Co., $38^{\circ} 55^{\prime} \mathrm{N}$ $78^{\circ} 49^{\prime} \mathrm{W}, 3 \mathrm{mi}$ NE Mathias, 29.VIII-25. IX.2006, Malaise trap, D. R. Smith (7 females, 1 male, USNMENT0097712600977131, 00993313-00993314 (USNM)). 
WV, Hardy Co., $38^{\circ} 55^{\prime} \mathrm{N} 78^{\circ} 49^{\prime} \mathrm{W}, 3 \mathrm{mi}$ NE Mathias, 7.VI-20.VI.2005, Malaise trap, D. R. Smith (1 female, USNMENT00993315 (USNM)). WV, Hardy Co., $38^{\circ} 55^{\prime} \mathrm{N} 78^{\circ} 49^{\prime} \mathrm{W}, 3 \mathrm{mi}$ NE Mathias, 8 . V-29.V.2008, Malaise trap, D. R. Smith (1 unknown, USNMENT00993559 (USNM)). WV, Hardy Co., $38^{\circ} 55^{\prime} \mathrm{N} 78^{\circ} 49^{\prime} \mathrm{W}, 3 \mathrm{mi}$ NE Mathias, 8.VII-25.VII.2005, D. R. Smith (11 females, USNMENT0099390800993918 (USNM)). WV, Hardy Co., $38^{\circ} 55^{\prime} \mathrm{N} 78^{\circ} 49^{\prime} \mathrm{W}, 3 \mathrm{mi}$ NE Mathias, 9. IX-2.X.2008, Malaise trap, D. R. Smith (2 females, USNMENT0099388500993886 (USNM)). WV, Hardy Co., Mathias, 26.VII-11.VIII.2005, D. R. Smith (2 males, USNMENT0099367000993671 (USNM)). WV, Hardy Co., Mathias, 8.VII-25.VII.2005, D. R. Smith (1 unknown, USNMENT00993623 (USNM)). Washington, 18.VII.1917, R. M. Fouts (1 female, USNMENT00977319 (USNM)). Washington, 21.VI.1917, R. M. Fouts (1 female, USNMENT00977362 (USNM)). Washington, 4.XII.1964, L. A. Kulp (1 male, USNMENT00977321 (USNM)). Washington, 6.VIII.1917, Fouts (4 females, USNMENT00977315-00977318 (USNM)). Washington, 9.VI.1917, R. M. Fouts (3 females, USNMENT00977320, 00977360-00977361 (USNM)). Washington, VI-1917, R. M. Fouts (1 female, USNMENT00977363 (USNM)). UNKNOWN: 6.IV.1918 (1 male, USNMENT00977020 (USNM)).

Comments.-Buffington and Scheffer (2008) recognized both Agrostocynips diastrophi and $A$. robusta as separate species. With the acquisition of additional specimens in the course of this research, it was determined that diastrophi and robusta represent opposite ends of a morphological spectrum. Specifically, traps in Kansas and Maryland yielded specimens of both morphotypes, as well as intermediates, in precisely the same location and over the same span of time. Hence, we recognize diastrophi as a junior synonym of robusta: syn. nov. The same applies to $A$. clavatus syn. nov. and $A$. enneatoma: syn. nov. This species is one of those clearly belonging in Zaeucoila with the revised circumscription, and thus the combination Zauecoila robusta n.comb. is made here.

Distribution.-Nearctic Region: Canada, United States and Northeastern Mexico (Buffington and Scheffer 2008). Neotropical region: Argentina, Brazil and St. Vincent (De Santis and Díaz 1975; De Santis et al. 1976). New country records: Anguilla, Chile, Colombia, Costa Rica, Cuba, Dominica, Ecuador, Guadeloupe, Guatemala, Jamaica, Martinique, Mexico, Panama, Peru, Saint Kitts and Nevis, Saint Vincent and The Grenadines and Trinidad and Tobago. New province records for Argentina: Jujuy, Mendoza, Neuquén and Tucuman.

Biology.-Hosts include Melanagromyza cunctanoides Blanchard (De Santis et al. 1976) and Liriomyza huidobrensis (Blanchard) (Díaz and Valladares 1979).

\section{Zaeucoila trianguligera Kieffer}

http://bioguid.osu.edu/xbiod_concepts/ 399055

$$
\text { (Figs. 76-81) }
$$

Zaeucoila trianguligera Kieffer, 1907: 91 (original description); Buffington, 2009: 185 (listed, host association).

Zaeucoila triangulilfera (incorrect subsequent spelling) Kieffer: Díaz and Gallardo, 1997: 39 (description).

Redescription.-Coloration with head, mesosoma, metasoma black to dark brown; legs with coxae and femora dark brown, rest yellow. Lateral margin of occiput defined by distinctly angled, raised, sharp carina. Vertical carina 

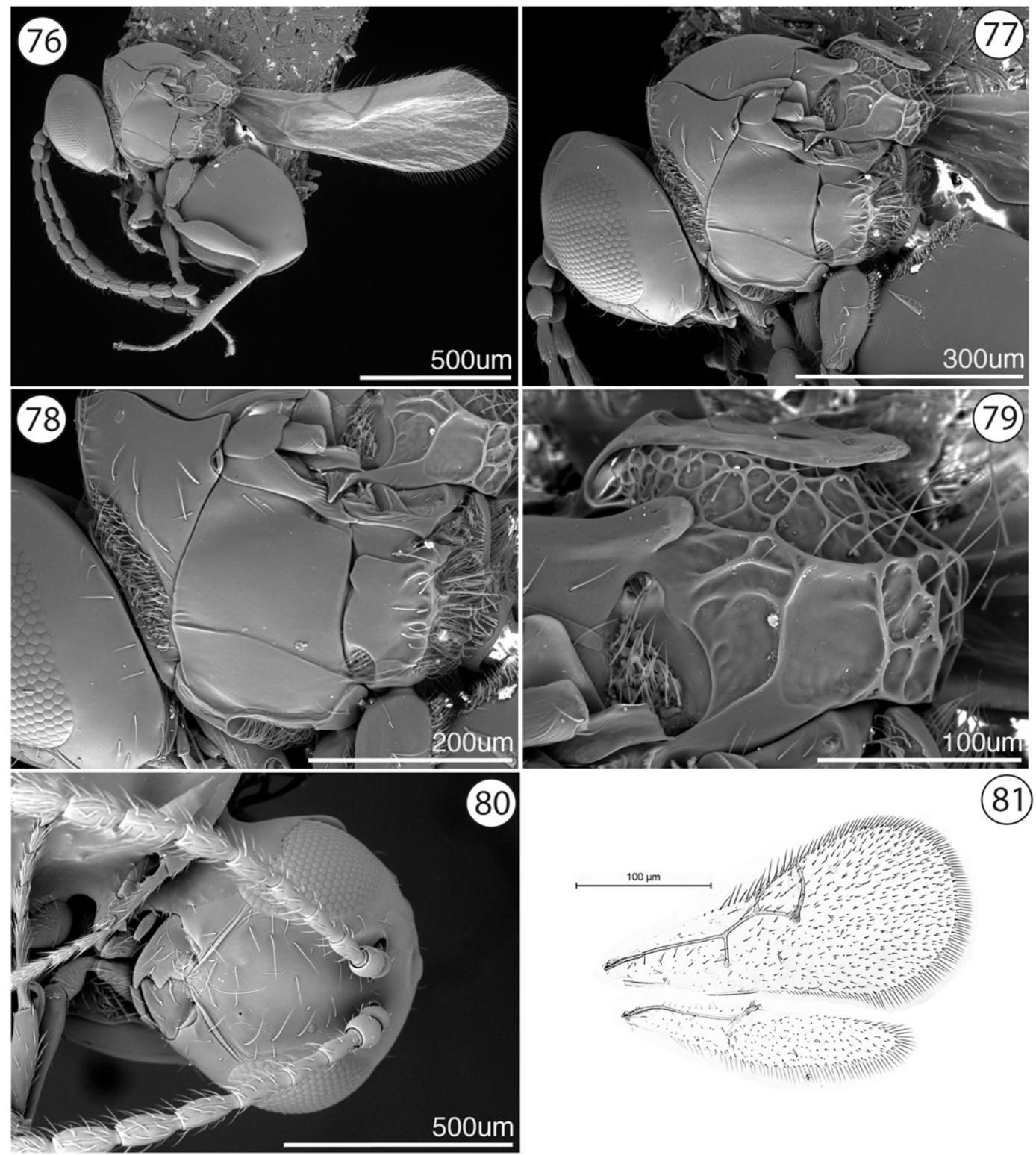

(81)

Plate 14, Figs. 76-81. Zaeucoila trianguligera Kieffer. 76, habitus, lateral view; 77, head and mesosoma, lateral view; 78, mesosoma, lateral view; 79, scutellum, lateral view; 80, head, anterior view; 81 , fore and hind wings.

adjacent to ventral margin of antennal socket present, leading to shallow inner orbital groove. Malar space adjacent to anterior articulation of mandible evenly rounded, smooth. Malar sulcus present, composed of single line. Orbital furrows rounded, ill defined, running from lateral edge of torulus to ventral margin of compound eye. Dorsal aspect of vertex smooth with a few short, scattered setae. Posterior aspect of vertex smooth. Hair punctures on lateral aspect 
of vertex absent. Placoidal sensilla present on F3-11. Pubescence on lateral surface of pronotum present, sparse, consisting of few short hairs. Ridges extending posteriorly from lateral margin of pronotal plate distinct but short, not extending to the dorsal margin of pronotum. Sculpture on mesoscutum absent, entire surface smooth, shiny. Median mesoscutal carina present, composed of anteriorly broad elevation, narrowing posteriorly, ending before midline of mesoscutum. Anterior admedial lines present, in conjunction with mesoscutal carina. Lateroventral mesopleural carina present, marking abrupt change of slope of mesopectus, terminating anteriorly at large, oblique notch. Mesopleural triangle present, gently impressed with distinct dorsal border, ventral border lacking; smooth. Dorsal surface of scutellum foveateareolet. Latero-ventral margin of scutellum posterior to auricula smooth ventrally, weakly rugulose dorsally. Subalar area abruptly broadened anteriorly, with an indicated longitudinal division. Pubescence present, but very sparse, along posterior and ventral margins of metapleuron; short and dense on propodeum. Pubescence of fore wing mesally sparse, gradually becoming denser distally. Coloration of wing absent, entire wing hyaline. Hair fringe along apical margin of fore wing present, with particularly longer setae along the mesal anterior margin. Inter propodeal carinae space moderately setose, with long, thin setae evenly filling the space; surface underneath smooth.

Diagnosis.-Due to the reduced state of the mesoscutellar carina, this species is most readily confused with $Z$. robusta. The clearest character for separating these species is the extent of the genal carina: in Z. triangulifera, complete, reaching the dorsal margin of the compound eye; in $Z$. robusta, reduced, only reaching the middle of the compound eye. Lighting is critical to see this character. Lastly, Z. robusta is known from North America north of Mexico, and $Z$. triangulifera is not.

Link to Distribution Map.-[http:// hol.osu.edu/map-full.html?id=399055]

Material Examined.-Holotype, unknown gender: NICARAGUA: Chinandega Dept., no date, Baker, CASE Type No. 10615 (deposited in CAS). Other material: (148 females, 63 males, 1 unknown). ARGENTINA: Corrientes Prov., Bella Vista, 2.X.1937 (1 female, USNMENT01223573 (MLPA)). Formosa Prov., S of Formosa, along river, RN 11, 60m, 26.III.2003, sweeping, J. Munro (1 male, USNMENT00977612 (USNM)). La Rioja Prov., El Duraznillo, 1.I-15.I.2003, Malaise trap, P. Fidalgo (1 female, USNMENT00977721 (USNM)). La Rioja Prov., Santa Cruz, 31.XII.2002, pan trap, P. Fidalgo (1 male, USNMENT00977716 (USNM)). Misiones Prov., nr. Loreto, wet forest, $77 \mathrm{~m}$, $27.34^{\circ} \mathrm{S} 55.53^{\circ} \mathrm{W}$, Santa Ana, 27. III.2003, sweeping, J. Munro (1 male, USNMENT00993767 (USNM)). Santiago del Estero Prov., 10km ENE Colonia Dora, Salado River, 17.XI-25. XI.1979, C. Vardy \& M. Vardy (1 female, USNMENT00970108 (BMNH)). BELIZE: Belize Dist., Airport Camp, II-1982, M. J. Grimshaw (1 male, USNMENT00970058 (BMNH)). Toledo Dist., Punta Gorda, 12.V-26.V.1982, Kelly \& George (1 female, 3 males, USNMENT00970060, 00970062, 00970065, 00970072 (BMNH)). Toledo Dist., Punta Gorda, 27.IV-7.V.1982, P. Kelley \& R. S. George (1 male, USNMENT00970106 (BMNH)). no date, reared (1 female, 1 male, USNMENT00993780-00993781 (USNM)). BRAZIL: MG, Ouro Preto, IV1954, N. L. H. Krauss (1 male, 
USNMENT00977574 (USNM)). RJ, Silva Jardim Mpio., Poço das Antas Biological Reserve, 1998, reared, Barbosa da Silva (1 female, USNMENT01223574 (MLPA)). SC, $27^{\circ} 11^{\prime} \mathrm{S} 52^{\circ} 23^{\prime} \mathrm{W}$, Nova Teutônia, 15.III.1937, F. Plaumann (1 unknown, USNMENT00969788 (BMNH)). SC, $27^{\circ} 11^{\prime} \mathrm{S} 52^{\circ} 23^{\prime} \mathrm{W}$, Nova Teutônia, 15. XII.1938, F. Plaumann (2 females, USNMENT00969767-00969768 (BMNH)). SC, $27^{\circ} 11^{\prime} \mathrm{S} 52^{\circ} 23^{\prime} \mathrm{W}$, Nova Teutônia, 22.IX.1935, F. Plaumann (1 male, USNMENT00969812 (BMNH)). SC, 2711'S $52^{\circ} 23^{\prime} \mathrm{W}$, Nova Teutônia, 22.IX.1949, F. Plaumann (1 male, USNMENT00969872 (BMNH)). SC, $27^{\circ} 11^{\prime} \mathrm{S} 52^{\circ} 23^{\prime} \mathrm{W}$, Nova Teutônia, 6.VI.1937, F. Plaumann (1 female, USNMENT00970128 (BMNH)). SC, $27^{\circ} 11^{\prime} \mathrm{S} 52^{\circ} 23^{\prime} \mathrm{W}$, Nova Teutônia, II-1937, F. Plaumann (12 females, USNMENT00969815, 00969819, 00969823, 00969825-00969826, 00969830, 00969833, 00969837-00969840, 00969844 (BMNH)). SC, $27^{\circ} 11^{\prime} \mathrm{S} 52^{\circ} 23^{\prime} \mathrm{W}$, Nova Teutônia, XI-1935, F. Plaumann (1 female, 1 male, USNMENT00969827, 00969836 (BMNH)). SC, $27^{\circ} \mathrm{S} 52.58^{\circ} \mathrm{W}$, Nova Teutônia, 24. III.1938, F. Plaumann (1 female, USNMENT00970027 (BMNH)). SC, $27^{\circ} \mathrm{S}$ $52.58^{\circ} \mathrm{W}$, Nova Teutônia, 3.VI.1938, F. Plaumann (1 female, USNMENT00969562 (BMNH)). COLOMBIA: Valle del Cauca Dept., by sugarcane field, $10 \mathrm{~km} \mathrm{~S}$ Cali, 1000m, 3.IV.1991, W. Eberhard \& C. Garcia (6 females, USNMENT00977675, 00977679-00977680, 00977685-00977687 (USNM)). COSTA RICA: Alajuela Prov., 5km W San Ramón, 1200m, VII-1997 (1 female, USNMENT00993778 (USNM)). Alajuela Prov., NE of Colonia Dos Ríos, La Selva Estate, 400m, 27.III.1988, Hanson (1 female, USNMENT00969963 (BMNH)). Alajuela Prov., Peninsula Path, 600m, $10^{\circ} 27^{\prime} \mathrm{N} 84^{\circ} 40^{\prime} \mathrm{W}$, Arenal Volcano National Park, 25.II.2003, J. Noyes (1 male, USNMENT00977973 (USNM)).
Alajuela Prov., Pilón Path, 600m, $10^{\circ} 27^{\prime} \mathrm{N}$ $84^{\circ} 43^{\prime} \mathrm{W}$, Arenal Volcano National Park, 26.II.2003, sweeping, J. Noyes (1 female, USNMENT00993830 (USNM)). Cartago Prov., Linda Vista Nursery, Dulce Nombre, 1300m, IX-1993, P. Hanson (1 female, USNMENT00977966 (USNM)). Guanacaste Prov., $13 \mathrm{~km}$ SE La Cruz, El Hacha Hill, 300m, no date, Janzen \& Gauld (1 female, USNMENT00993829 (USNM)). Guanacaste Prov., 20km SW Cañas, Enrique Jimenez Nuñez Experimental Station, 5.XI17.XI.1991, Malaise trap, A. S. Menke (3 females, 7 males, USNMENT0097757500977579, 00977582, 00977858-00977859, 00993951, 00993953 (USNM)). Guanacaste Prov., Cacao Hill, Mengo Station, Guanacaste National Park, 1000m, V-1988, Gauld \& Jansen (1 female, 2 males, USNMENT00969994, 00969996-00969997 (BMNH)). Guanacaste Prov., H.1.O, Santa Rosa National Park, 300m, 1.VI22.VI.1985, Malaise trap, D. Janzen \& I. Gauld (1 female, 1 male, USNMENT00977658, 00993590 (USNM)). Guanacaste Prov., H.1.O, Santa Rosa National Park, 300m, 10.I-31.I.1987, Malaise trap, D. Janzen \& I. Gauld (1 female, 1 male, USNMENT0097799300977994 (USNM)). Guanacaste Prov., H.1.O, Santa Rosa National Park, 300m, 11.V-1.VI.1985, Malaise trap, D. Janzen \& I. Gauld (2 females, USNMENT01029112-01029113 (USNM)). Guanacaste Prov., H.1.O, Santa Rosa National Park, 300m, 14.VI-5.VII.1985, Malaise trap, D. Janzen \& I. Gauld (6 females, 1 male, USNMENT00977638, 00977645-00977647, 00993579-00993581 (USNM)). Guanacaste Prov., H.1.O, Santa Rosa National Park, 300m, 14.VIII-6. IX.1986, Malaise trap, D. Janzen \& I. Gauld (3 females, USNMENT01029033-01029035 (USNM)). Guanacaste Prov., H.1.O, Santa Rosa National Park, 300m, 18.X-8.XI.1986, Malaise trap, D. Janzen \& I. Gauld (2 females, USNMENT01029052, 01029054 
(USNM)). Guanacaste Prov., H.1.O, Santa Rosa National Park, 300m, 22.VI13.VII.1985, Malaise trap, D. Janzen \& I. Gauld (1 male, USNMENT01029024 (USNM)). Guanacaste Prov., H.1.O, Santa Rosa National Park, 300m, 23.III13.IV.1986, Malaise trap, D. Janzen \& I. Gauld (1 female, USNMENT01029030 (USNM)). Guanacaste Prov., H.1.O, Santa Rosa National Park, 300m, 26. VII-14.VIII.1986, Malaise trap, D. Janzen \& I. Gauld (2 females, 2 males, USNMENT01029043-01029046 (USNM)). Guanacaste Prov., H.1.O, Santa Rosa National Park, 300m, 27.IV-11.V.1985, Malaise trap, D. Janzen \& I. Gauld (1 female, USNMENT00993592 (USNM)). Guanacaste Prov., H.1.O, Santa Rosa National Park, 300m, 27.IX-18.X.1986, Malaise trap, D. Janzen \& I. Gauld (1 female, USNMENT01029074 (USNM)). Guanacaste Prov., H.1.O, Santa Rosa National Park, 300m, 29.XI-20.XII.1986, Malaise trap, D. Janzen \& I. Gauld (1 female, 2 males, USNMENT0102905601029058 (USNM)). Guanacaste Prov., H.1.O, Santa Rosa National Park, 300m, 5.VII-26.VII.1986, Malaise trap, D. Janzen \& I. Gauld (1 female, USNMENT01029077 (USNM)). Guanacaste Prov., H.1.O, Santa Rosa National Park, 300m, 6.IX-27.IX.1986, Malaise trap, D. Janzen \& I. Gauld (1 female, USNMENT00977665 (USNM)). Guanacaste Prov., H.2.C, Santa Rosa National Park, 300m, 1.VI-27.VI.1985, Malaise trap, D. Janzen \& I. Gauld (1 female, USNMENT00977661 (USNM)). Guanacaste Prov., H.2.C, Santa Rosa National Park, 300m, 10.I-31.I.1987, Malaise trap, D. Janzen \& I. Gauld (1 female, USNMENT01029019 (USNM)). Guanacaste Prov., H.2.C, Santa Rosa National Park, 300m, 11.V-1.VI.1985, Malaise trap, D. Janzen \& I. Gauld (1 female, USNMENT01029041 (USNM)). Guanacaste Prov., H.2.C, Santa Rosa National Park, 300m, 13.IV-4.V.1986, Malaise trap, D. Janzen \& I. Gauld (2 females, USNMENT01029027, 01029029 (USNM)). Guanacaste Prov., H.2.C, Santa Rosa National Park, 300m, 14.VI-5.VII.1986, Malaise trap, D. Janzen \& I. Gauld (1 male, USNMENT01029023 (USNM)). Guanacaste Prov., H.2.C, Santa Rosa National Park, 300m, 14.VIII-6. IX.1986, Malaise trap, D. Janzen \& I. Gauld ( 2 females, USNMENT01029018, 01029021 (USNM)). Guanacaste Prov., H.2.C, Santa Rosa National Park, 300m, 17.IV-27.IV.1985, Malaise trap, D. Janzen \& I. Gauld (1 male, USNMENT00977996 (USNM)). Guanacaste Prov., H.2.C, Santa Rosa National Park, 300m, 21.II-14.III.1987, Malaise trap, D. Janzen \& I. Gauld (1 female, USNMENT01029050 (USNM)). Guanacaste Prov., H.2.C, Santa Rosa National Park, 300m, 22.VI-13.VII.1985, Malaise trap, D. Janzen \& I. Gauld (1 female, USNMENT00977979 (USNM)). Guanacaste Prov., H.2.C, Santa Rosa National Park, 300m, 23.III.1986, Malaise trap, D. Janzen \& I. Gauld (1 female, USNMENT01029025 (USNM)). Guanacaste Prov., H.2.C, Santa Rosa National Park, 300m, 24.V-14.VI.1986, Malaise trap, D. Janzen \& I. Gauld (1 female, USNMENT00977660 (USNM)). Guanacaste Prov., H.2.C, Santa Rosa National Park, 300m, 26.VII-14.VIII.1986, Malaise trap, D. Janzen \& I. Gauld (1 female, USNMENT00977648 (USNM)). Guanacaste Prov., H.2.C, Santa Rosa National Park, 300m, 27.IX-18.X.1986, Malaise trap, D. Janzen \& I. Gauld (1 male, USNMENT01029042 (USNM)). Guanacaste Prov., H.2.C, Santa Rosa National Park, 300m, 31.I-21.II.1987, Malaise trap, D. Janzen \& I. Gauld (1 female, USNMENT01029105 (USNM)). Guanacaste Prov., H.2.C, Santa Rosa National Park, 300m, 5.VII-26.VII.1986, Malaise trap, D. Janzen \& I. Gauld (1 
female, USNMENT00977989 (USNM)). Guanacaste Prov., H.2.C, Santa Rosa National Park, 300m, 6.IX-27.IX.1986, Malaise trap, D. Janzen \& I. Gauld (1 female, USNMENT01029036 (USNM)). Guanacaste Prov., H.3.O, Santa Rosa National Park, 300m, 13.VII-3. VIII.1985, Malaise trap, D. Janzen \& I. Gauld (1 female, USNMENT01029093 (USNM)). Guanacaste Prov., H.3.O, Santa Rosa National Park, 300m, 14.VI5.VII.1986, Malaise trap, D. Janzen \& I. Gauld (10 females, 2 males, USNMENT00977630-00977631, 0097763300977637, 00993574-00993577, 00993582 (USNM)). Guanacaste Prov., H.3.O, Santa Rosa National Park, 300m, 14. VIII-6.IX.1985, Malaise trap, D. Janzen \& I. Gauld (1 female, 2 males, USNMENT00993568, 00993570-00993571 (USNM)). Guanacaste Prov., H.3.O, Santa Rosa National Park, 300m, 18.X8.XI.1986, Malaise trap, D. Janzen \& I. Gauld (2 females, USNMENT0102910701029108 (USNM)). Guanacaste Prov., H.3.O, Santa Rosa National Park, 300m, 2.III-23.III.1986, Malaise trap, D. Janzen \& I. Gauld ( 1 female, USNMENT00977662 (USNM)). Guanacaste Prov., H.3.O, Santa Rosa National Park, 300m, 20.XII-10.I.1987, Malaise trap, D. Janzen \& I. Gauld (1 female, 1 male, USNMENT00977655, 00977657 (USNM)). Guanacaste Prov., H.3.O, Santa Rosa National Park, 300m, 20.XII.1986, Malaise trap, D. Janzen \& I. Gauld (3 females, USNMENTO0977656, 00977983, 00993599 (USNM)). Guanacaste Prov., H.3.O, Santa Rosa National Park, 300m, 23.VI13.VII.1985, Malaise trap, D. Janzen \& I. Gauld (1 female, USNMENT00977663 (USNM)). Guanacaste Prov., H.3.O, Santa Rosa National Park, 300m, 26.VII14.VIII.1986, Malaise trap, D. Janzen \& I. Gauld $(6$ females, 1 male, USNMENT00977649, 00977978, 0099358300993584, 00993586-00993587, 00993602
(USNM)). Guanacaste Prov., H.3.O, Santa Rosa National Park, 300m, 26.X16.XI.1985, Malaise trap, D. Janzen \& I. Gauld (1 female, USNMENT01029099 (USNM)). Guanacaste Prov., H.3.O, Santa Rosa National Park, 300m, 27.IV11.V.1985, Malaise trap, D. Janzen \& I. Gauld (2 males, USNMENT00993591, 00993593 (USNM)). Guanacaste Prov., H.3.O, Santa Rosa National Park, 300m, 27.IX-18.X.1986, Malaise trap, D. Janzen \& I. Gauld (5 females, USNMENT00977641-00977644, 00993601 (USNM)). Guanacaste Prov., H.3.O, Santa Rosa National Park, 300m, 5.VII26.VII.1986, Malaise trap, D. Janzen \& I. Gauld (1 female, 2 males, USNMENT00977639-00977640, 00993594 (USNM)). Guanacaste Prov., H.4.C, Santa Rosa National Park, 300m, 1.VI22.VI.1985, Malaise trap, D. Janzen \& I. Gauld (1 female, USNMENT01029060 (USNM)). Guanacaste Prov., H.4.C, Santa Rosa National Park, 300m, 18.X8.XI.1986, Malaise trap, D. Janzen \& I. Gauld (1 female, USNMENT00977999 (USNM)). Guanacaste Prov., H.4.C, Santa Rosa National Park, 300m, 21.II14.III.1987, Malaise trap, D. Janzen \& I. Gauld (2 females, USNMENT01029009, 01029015 (USNM)). Guanacaste Prov., H.4.C, Santa Rosa National Park, 300m, 23.III-13.IV.1986, Malaise trap, D. Janzen \& I. Gauld (1 female, USNMENT01029059 (USNM)). Guanacaste Prov., H.4.C, Santa Rosa National Park, 300m, 27.IV-11.V.1985, Malaise trap, D. Janzen \& I. Gauld (1 female, USNMENT01029090 (USNM)). Guanacaste Prov., H.4.C, Santa Rosa National Park, 300m, 27.IX-18.X.1986, Malaise trap, D. Janzen \& I. Gauld (1 female, USNMENT00977982 (USNM)). Guanacaste Prov., SE.5.O, Santa Rosa National Park, 300m, 8.II-2.III.1986, Malaise trap, D. Janzen \& I. Gauld (1 female, USNMENT00977984 (USNM)). Guanacaste 
Prov., SE.6.C, Santa Rosa National Park, 300m, 13.IV-4.V.1986, Malaise trap, D. Janzen \& I. Gauld (1 female, USNMENT01029032 (USNM)). Guanacaste Prov., SE.6.C, Santa Rosa National Park, 300m, 31.I-21.II.1987, Malaise trap, D. Janzen \& I. Gauld (1 female, USNMENT00977990 (USNM)). Guanacaste Prov., SE.8.C, Santa Rosa National Park, 300m, 8.II-2.III.1986, Malaise trap, D. Janzen \& I. Gauld (1 female, USNMENT01029026 (USNM)). Guanacaste Prov., dry forest, Barra Honda National Park, 200m, V-1988, Gauld \& Mitchell (1 female, 2 males, USNMENT0096992200969923, 00969926 (BMNH)). Limón Prov., Cahuita, 23.XII-25.XII.1988, Malaise trap, A. S. Menke (2 females, USNMENT00977867, 00993956 (USNM)). Puntarenas Prov., Puerto Jiménez, II1992, P. Hanson (1 female, USNMENT00977740 (USNM)). San José Prov., Escazú Canton, San Antonio de Escazú, 1300m, II-1989, W. Eberhard (1 male, USNMENT00969989 (BMNH)). San José Prov., Escazú Canton, San Antonio de Escazú, 1300m, IV-1987, W. Eberhard (1 female, USNMENT00969953 (BMNH)). San José Prov., Escazú Canton, San Antonio de Escazú, 1500m, 3. VI.1987, P. Hanson (1 female, USNMENT00969983 (BMNH)). San José Prov., Moravia Canton, 6km NE San Jerónimo, Braulio Carrillo Highway, 1500m, 12.V.1988, Hanson (1 male, USNMENT00969957 (BMNH)). EL SALVADOR: La Libertad Dept., Quezaltepeque, 500m, 5.VII.1963, D. Q. Cavagnaro \& M. E. Irwin (1 female, USNMENT00977456 (CAS)). La Libertad Prov., Nueva San Salvador (Santa Tecla), 3.X.1965, N. L. H. Krauss (1 female, USNMENT00969849 (BMNH)). GUATEMALA: Sacatepéquez Dept., Sumpango, $14^{\circ} 40^{\prime} 17^{\prime \prime} \mathrm{N} \quad 90^{\circ} 43^{\prime} 11^{\prime \prime} \mathrm{W}$, Durwest Farm, 10.III-16.III.2007, M. Hoddle (1 female, USNMENT00993835
(USNM)). HONDURAS: Atlántida Dept., La Ceiba, 27.VIII.1916, F. J. Dyer (1 female, USNMENT00977566 (USNM)). MEXICO: CHIS, El Sumidero Canyon, 16.VII-17.VII.1984, G. Gordh (1 male, USNMENT00977438 (UCRC)). CHIS, Palenque, 23.VII.1984, G. Gordh (5 males, USNMENT00977431-00977434, 00977436 (UCRC)). CHIS, hwy 195, 90/ 064, 8.5mi N Ixhuatán (Ishuatan), 1200ft, 14.VIII.1990, J. B. Woolley (1 female, USNMENT00977573 (USNM)). SLP, 5mi N Tamazunchale, 22.XII.1948, E. S. Ross (1 male, USNMENT00977449 (CAS)). SLP, 7mi S Ciudad Valles, 300ft, $21^{\circ} 54^{\prime} \mathrm{N} 98^{\circ} 57^{\prime} \mathrm{W}$, El Bonito, 19.XII.1970, P. H. Arnaud \& M. Arnaud (1 male, USNMENT00977448 (CAS)). SON, Alamos, 25.II.1963, P. H. Arnaud (1 female, USNMENT00977447 (CAS)). TAMPS, 1986/ 002, 5mi W Gómez Farías, 20.III.1986, Woolley \& Zolnerowich (1 female, USNMENT00977384 (TAMU)). TAMPS, 5mi W Gómez Farías, 20.V.1986, R. Wharton (1 male, USNMENT00977408 (TAMU)). TAMPS, 6.5mi NW Ciudad Victoria, La Libertad Canyon, 21.III.1986, G. Zolnerowich (1 female, USNMENT00977383 (TAMU)). TAMPS, $8.2 \mathrm{~km}$ E Ciudad Victoria, MEX-70, 3.VII.1986, Zolnerowich \& Trevino (1 female, 1 male, USNMENT00977406-00977407 (TAMU)). TAMPS, 97km E Ciudad Victoria, MEX-70, 3.VII.1986, G. Zolnerowich \& R. Trevino (5 females, 2 males, USNMENTO097737000977371, 00977374-00977377, 00977379 (TAMU)). VER, 97/015, 0.7mi N Jilotepec, 3680ft, 14.VI.1997, screen sweeping, L. A. Wilson \& J. B. Woolley ( 3 females, 2 males, USNMENT00977409-00977412, 00977415 (TAMU)). VER, Veracruz, XI-1963, N. L. H. Krauss (1 male, USNMENT00977865 (USNM)). PANAMA: Bocas del Toro Prov., $8.8 \mathrm{~km}$ W Rambaia, 2001/028, 35m, $08^{\circ} 59^{\prime} 04^{\prime \prime} \mathrm{N} 82^{\circ} 13^{\prime} 57^{\prime \prime} \mathrm{W}$, La Gloria River, 8. I.2001, screen sweeping, M. Yoder \& J. B. Woolley (1 female, USNMENT00977669 
(USNM)). PARAGUAY: San Pedro Dept., Ypané River, Cororó, 5.XII-9.XII.1983, Malaise trap, M. Wasbauer (1 female, USNMENT01081066 (USNM)). TRINIDAD AND TOBAGO: Tobago Isl., Courland River, Turtle Beach, 19.X.1977, S. L. Ward (1 female, USNMENT00970055 (BMNH)). VENEZUELA: Mérida St., 7km E Tabay, Mucuy Fish Hatchery, 6600ft, 10. II-13.II.1978, blacklight trap, J. B. Heppner (1 female, USNMENTO0993989 (USNM)). Distribution.-Neotropical region: Argentina, Brazil, Nicaragua and Panama (Kieffer 1907; Díaz and Gallardo 1997; Fontal and Nieves-Aldrey 2004; Marchiori et al. 2004). New state record for Brazil: Rio de Janeiro.

Biology.-Recorded hosts include Liriomyza sp. on Lactuca sativa L., Amauromyza maculosa (Malloch) and Agromyza fusca Spencer (in Buffington 2009). Calycomyza sp. is reported here as a new agromyzid host record (based on label data).

\section{Zaeucoila unicarinata Ashmead}

http://bioguid.osu.edu/xbiod_concepts/ 387823

(Figs. 82-87)

Zaeucoila unicarinata Ashmead: Dalla Torre and Kieffer, 1910: 103 (description); Weld, 1921: 445-446 (original description); Díaz and Gallardo, 1997: 38 (description); Buffington, 2009: 185 (listed).

Redescription.-Coloration with head, mesosoma, metasoma black to dark brown; legs yellow-orange. Lateral margin of occiput defined by scalloped, sharp carina. Vertical carina adjacent to ventral margin of antennal socket present, leading to deep inner orbital groove. Malar space adjacent to anterior articulation of mandible with distinct pyramidal protuberance, smooth. Malar sulcus present, composed of series of small striae dorsally, leading to single line ventrally. Orbital furrows rounded, divergent ledges running from antennal sockets to dorsal end of malar sulcus. Dorsal aspect of vertex smooth. Posterior aspect of vertex smooth. Hair punctures on lateral aspect of vertex absent. Placoidal sensilla present on F 2-11. Pubescence on lateral surface of pronotum present, sparse, with distinct row behind pronotal plate. Ridges extending posteriorly from lateral margin of pronotal plate present, variable, short to medium long, in some cases running to anterior margin of mesoscutum. Sculpture on mesoscutum absent, entire surface smooth, shiny. Median mesoscutal carina present, composed of anteriorly broad elevation, narrowing posteriorly, ending at midline of mesoscutum. Anterior admedial lines present, in conjunction with mesoscutal carina. Lateroventral mesopleural carina present, marking abrupt change of slope of mesopectus, terminating anteriorly at large, oblique notch. Mesopleural triangle present, gently impressed with distinct dorsal border, ventral border lacking; smooth. Dorsal surface of scutellum laterally with distinct ridges radiating from plate, posteriorly with deep fovea and ridges. Latero-ventral margin of scutellum posterior to auricula deeply foveate. Subalar area slightly broadened anteriorly, with an indicated longitudinal division. Pubescence consisting of few hairs on posterior part of metaplueron, dense hair on propodeum. Pubescence of fore wing mesally sparse, gradually becoming denser distally. Coloration of wing variable mesally, ranging from very light blotchy infuscation to restricted infuscation immediately adjacent to the basalis the ventral bulb of basalts; rest of wing hyaline. Hair fringe along apical margin of fore wing present, with particularly longer setae along the 

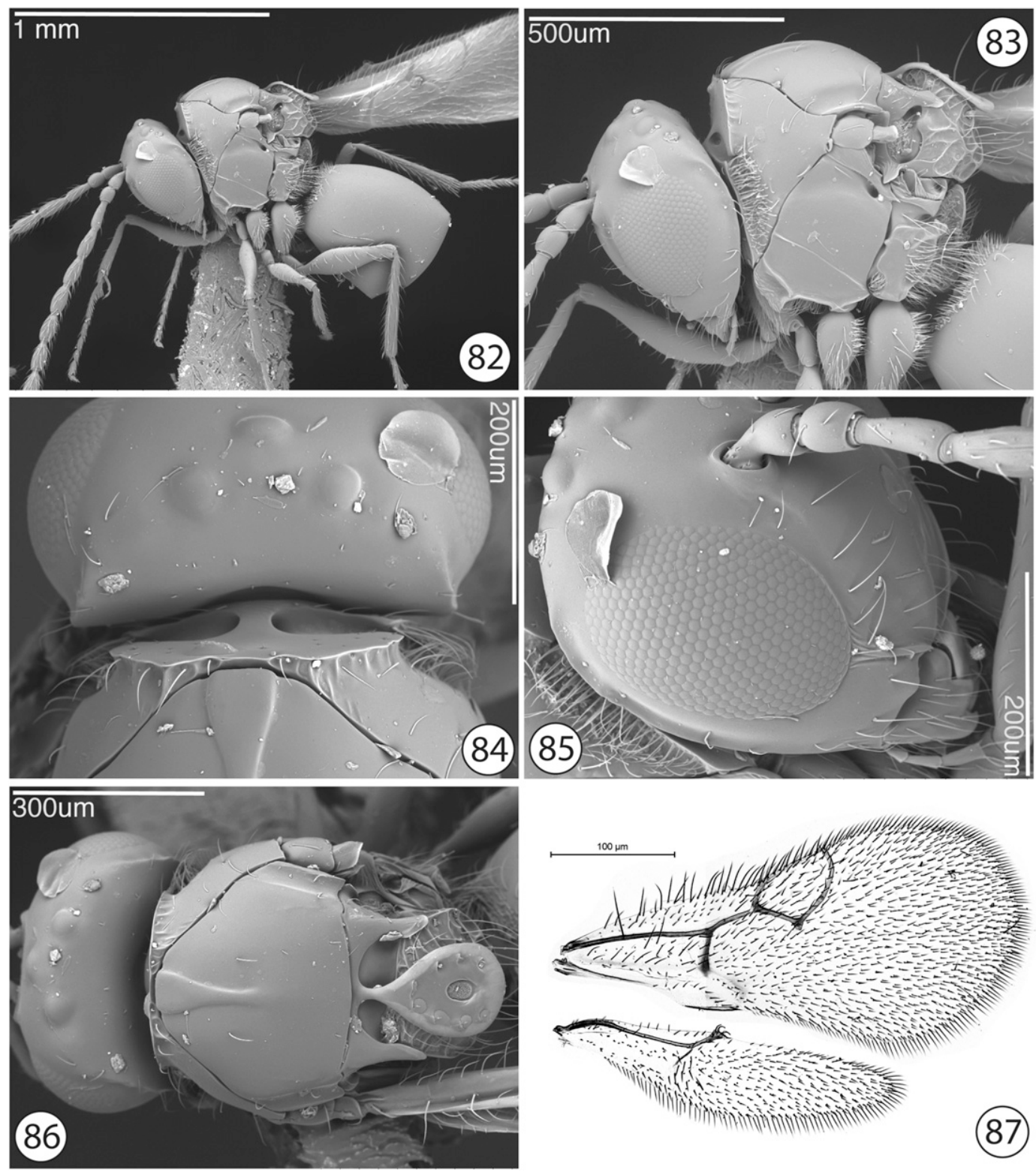

Plate 15, Figs. 82-87. Zaeucoila unicarinata Ashmead. 74, habitus, lateral view; 75, head and mesosoma, dorso-lateral view; 76, head and mesosoma, antero-dorsal view; 77, scutellum, dorso-lateral view; 78, head, antero-lateral view; 79, fore and hind wings.

mesal anterior margin. Inter propodeal carinae space densely setose.

Diagnosis. - The distinctly scalloped genal carina of the head, and complete and readily visible mesoscutal carina of the mesoscutum, separate this species from $Z$. lignys, which is the species Z. unicarinata is most readily confused with. Some $Z$. flavipes look similar to $Z$. unicarinata, but in Z. flavipes, the two pairs of hairs are distinctly long, and the only hairs present on the mesoscutum; in Z. unicarinata, if 


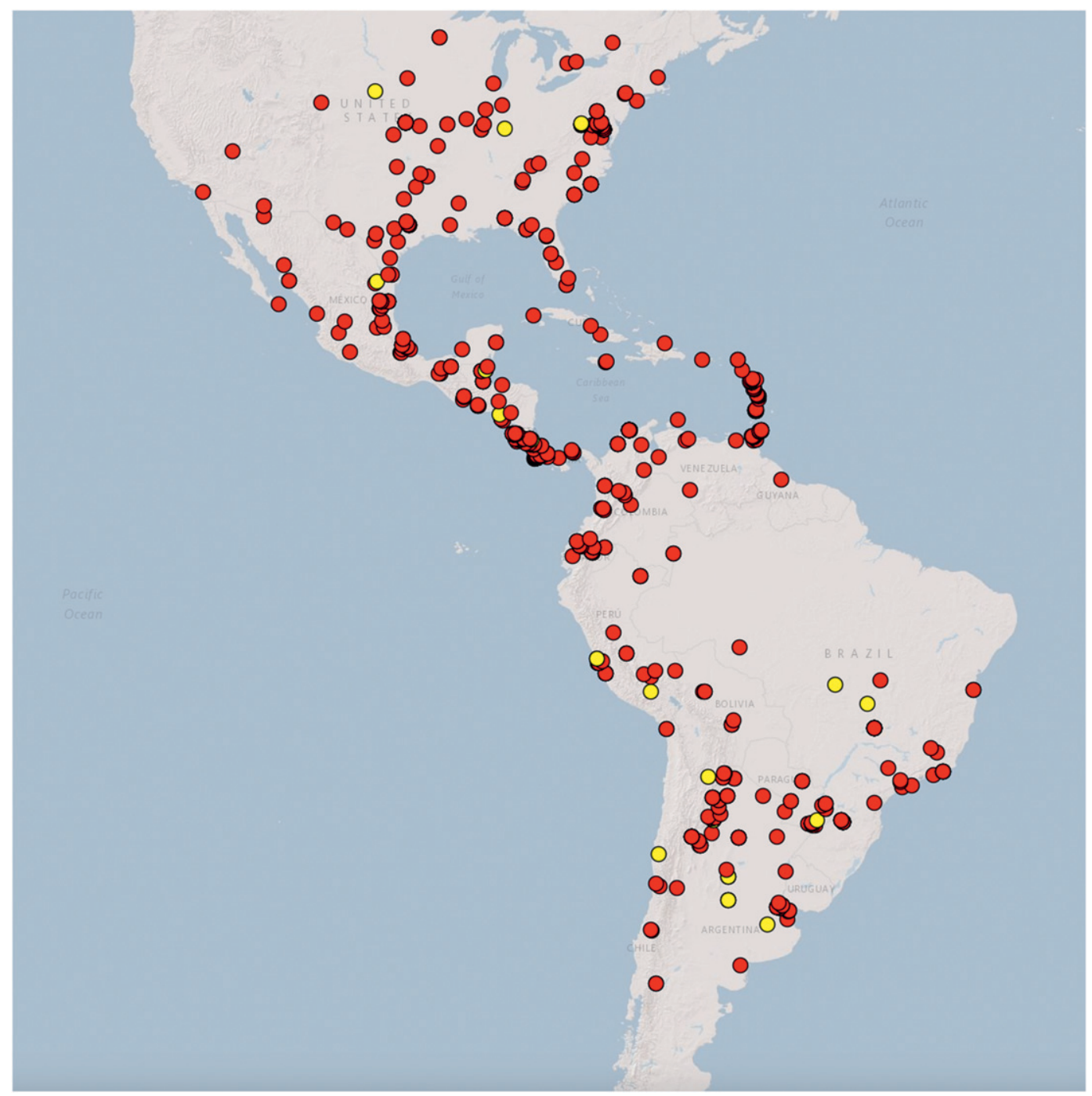

Fig. 88. Distribution map of Zaeucoila species.

there are paired hairs, they are not very long and are typically present with other hairs.

Link to Distribution Map.-[http:// hol.osu.edu/map-full.html?id=387823]

Material Examined.-Holotype, female: BRAZIL: RJ, Rio de Janeiro, VIII, USNM Type No. 23650 (deposited in USNM). Other material: (24 females, 5 males) ARGENTINA: Buenos Aires Prov., Villarino Dept., Mayor Buratovich, $39^{\circ} 16^{\prime} 12.12^{\prime \prime} \mathrm{S} \quad 62^{\circ} 44^{\prime} 00.37^{\prime \prime} \mathrm{W}$, Colonia
La Julita, 21.XI.2012, reared, Caracotche (1 male, USNMENT01223575 (MLPA)). Misiones Prov., Loreto, 10. VIII.1930, Ogloblin (1 female, USNMENT01223578 (MLPA)). Misiones Prov., Loreto, 14.III.1930 (1 male, USNMENT01223576 (MLPA)). Misiones Prov., Loreto, 14.III.1930, Ogloblin (1 male, USNMENT01223577 (MLPA)). Misiones Prov., Loreto, 18. I.1931, Ogloblin (1 male, USNMENT01223581 (MLPA)). Misiones 
Prov., Loreto, 20.V.1930, Ogloblin (1 female, USNMENT01223582 (MLPA)). Misiones Prov., Loreto, 5.IV.1931, Ogloblin (1 female, USNMENT01223583 (MLPA)). Misiones Prov., Loreto, X-1930, Ogloblin (1 female, USNMENT01223579 (MLPA)). Misiones Prov., Loreto, no date, Ogloblin (1 female, USNMENT01223580 (MLPA)). BRAZIL: BA, Centro de Pesquisas do Cacau (CEPEC), Itabuna, 24.II-27.II.1983, F. P. Benton (1 female, USNMENT00969577 (BMNH)). GO, Itumbiara, 11.IV.2002, Marchiori (1 female, USNMENT01223584 (MLPA)). SC, $27^{\circ} 11^{\prime} \mathrm{S} 52^{\circ} 23^{\prime} \mathrm{W}$, Nova Teutônia, 11.VII.1935, F. Plaumann (1 female, USNMENT00969814 (BMNH)). SC, $27^{\circ} 11^{\prime} \mathrm{S} 52^{\circ} 23^{\prime} \mathrm{W}$, Nova Teutônia, 18. X.1938, F. Plaumann (1 female, USNMENT00969764 (BMNH)). SC, 2711'S $52^{\circ} 23^{\prime} \mathrm{W}$, Nova Teutônia, 19.XI.1937, F. Plaumann (1 female, USNMENT00970012 (BMNH)). SC, $27^{\circ} 11^{\prime} \mathrm{S} 52^{\circ} 23^{\prime} \mathrm{W}$, Nova Teutônia, 20.V.1937, F. Plaumann (1 female, USNMENT00969868 (BMNH)). SC, $27^{\circ} 11^{\prime} \mathrm{S} 52^{\circ} 23^{\prime} \mathrm{W}$, Nova Teutônia, 25. VI.1937, F. Plaumann (1 female, USNMENT00969876 (BMNH)). SC, 2711'S $52^{\circ} 23^{\prime} \mathrm{W}$, Nova Teutônia, 4.IV.1938, F. Plaumann (1 female, USNMENT00970014 (BMNH)). SC, $27^{\circ} 11^{\prime} \mathrm{S} 52^{\circ} 23^{\prime} \mathrm{W}$, Nova Teutônia, 7.VIII.1935, F. Plaumann (1 female, USNMENT00969845 (BMNH)). SC, $27^{\circ} 11^{\prime} \mathrm{S} 52^{\circ} 23^{\prime} \mathrm{W}$, Nova Teutônia, 9. VIII.1935, F. Plaumann (2 females, USNMENT00969810, 00969834 (BMNH)). SC, $27^{\circ} 11^{\prime} \mathrm{S} 52^{\circ} 23^{\prime} \mathrm{W}$, Nova Teutônia, XI1935, F. Plaumann (1 female, 1 male, USNMENT00969811, 00969813 (BMNH)). $\mathrm{SC}, 27^{\circ} \mathrm{S} 52.58^{\circ} \mathrm{W}$, Nova Teutônia, 2.VI.1938, F. Plaumann (1 female, USNMENT00970036 (BMNH)). SC, $27^{\circ} \mathrm{S} 52.58^{\circ} \mathrm{W}$, Nova Teutônia, 3. VI.1938, F. Plaumann (2 females, USNMENT00969568-00969569 (BMNH)). SC, $27^{\circ} \mathrm{S} 52.58^{\circ} \mathrm{W}, \quad$ Nova Teutônia, 7. IV.1938, F. Plaumann (1 female, USN-
MENT00970028 (BMNH)). COSTA RICA: Guanacaste Prov., 20km SW Cañas, Enrique Jimenez Nuñez Experimental Station, 5.XI-17.XI.1991, Malaise trap, A. S. Menke (1 female, USNMENT00993945 (USNM)). Guanacaste Prov., SE.5.O, Santa Rosa National Park, 300m, 1.VI-22.VI.1985, Malaise trap, D. Janzen \& I. Gauld (1 female, USNMENT01223595 (USNM)). VENEZUELA: Zulia St., $51 \mathrm{~km}$ SW Machiques, Los Ángeles del Tucuco, 1. VI-24.VI.1983, E. Inciarte (1 female, USNMENT01223585 (MALUZ)).

Distribution.-Neotropical region: Argentina, Brazil, and Panama, (Ashmead 1903a, Díaz and Gallardo 1997, Fontal and Nieves-Aldrey 2004, Gallardo and Dughetti 2013). New country record for Costa Rica and Venezuela; new state record for Brazil: Goias.

Biology.-Recorded hosts include Liriomyza sativa Blanchard on Cucumis sativus L. (Lorini and Foerster 1985), Liriomyza sp. on Allium cepa L. (Gallardo and Dughetti 2013).

\section{Conclusion}

The geographic distribution of Zaeucoila as circumscribed now range from southern Canada to the north, through Central and South America, and to Patagonia in Argentina, thus expanding the general distribution of Zaeucoilini further south than previously recorded (Buffington 2009). This is not surprising given the number of specimens examined in this study; additional studies on other zaeucoiline genera may indeed expand the general distribution of the tribe even further. A striking apparent distribution 'hole' is Brazil, as specimens from this country were difficult to obtain for this study. It is likely Zaeucoila species are present throughout Brazil, and there are also likely undescribed species awaiting discovery. Interestingly, Zaeucoila is the 
only zaeucoiline genus that is commonly observed in North America north of Mexico. Buffington (2009), treating the North American species of Zaeucoila under Agrostocynips, speculated that agroymyzid hosts in North America are dominated by diglyphosematine Eucoilinae, the putative sister-group of Zaueucoilinae. A follow up divergence analysis by Buffington et al. (2015) suggested the zaeucoiline lineage is much younger than the diglyphosematine lineage, and speculate that Zaeucoilinae emerged within the Neotropical Region, with a single species (Z. robusta) able to carve out niche in North America. Future research may yield how these two major lineages of eucoilines intermingle in central and northern Mexico, and possibly how the two lineages avoid competing for similar (or identical) hosts.

The new country and provinces/states records given in this paper for previously described species are the following: for Zaeucoila grenadensis: Brazil, Ecuador, and Peru; for $Z$. incompleta: Belize, Bolivia, Colombia, Costa Rica, Dominica, Dominican Republic, Ecuador, El Salvador, Guadeloupe, Guatemala, Guyana, Honduras, Mexico, Nicaragua, Peru, Puerto Rico, Saint Vincent and the Grenadines and Trinidad and Tobago; new province records for Argentina: Buenos Aires, Cordoba, Salta and Tucuman; new state records for Brazil: Minas Gerais, Rio de Janeiro and Sao Paulo; Zaeucoila robusta: Anguilla, Chile, Colombia, Costa Rica, Cuba, Dominica, Ecuador, Guadeloupe, Guatemala, Jamaica, Martinique, Mexico, Panama, Peru, Saint Kitts and Nevis, Saint Vincent and The Grenadines and Trinidad and Tobago; new province records for Argentina: Jujuy, Mendoza, Neuquén and Tucuman; for $Z$. triangulifera: new state record for Brazil: Rio de Janeiro; for Z. unicarinata: Costa Rica and Venezuela; new state record for Brazil: Goias.
Nearctic species of Zaeucoila have been reared from agromyzids in Agromyza Fallén on Panicum L. (Poaceae), Liriomyza huidobrensis on commercial Capsicum (Buffington, pers. obsv.) and Phytomyza Fallén on Ilex cassine L. and Ilex myrtifolia Walter (Buffington 2009). Neotropical species of this genus have been recorded from Liryomyza, Melanagromyza, Nemorimyza Frey, Amauromyza Hendel, Agromyza Fallén and Calycomyza Hendel. The new hosts records given in this paper are the following for Zaeucoila grenadensis: Calycomyza hiptidis Spencer, Calycomyza servilis Spencer, Calycomyza eupatorivora Spencer, and an unidentified Calycomyza species; for Zaeucoila incompleta: Calycomyza servilis and Liryomyza huidobrensis; for $Z$. trianguligera: an undescribed species of Calycomyza. As many species of agrozymids are considered pestiferous, we hope this treatment of Zaeucoila will aid in endeavors focused on natural control of these pests.

\section{ACKNOWLEDGMENTS}

The authors wish to thank J. M. Maes, C. Marchiori and R. Monteiro for the donation of specimens. We would also like to thank the curators at all of the lending instutions listed under the Materials and Methods, especially Bob Zuparko (CASC), Doug Yanega (UCRC) and David Notton (NHM), all of whom extended loans for this project. We also extend our gratitude to Taina Litwak (Systematic Entomology Laboratory, ARS-USDA) for the amazing digital painting of Zaeucoila robusta in Figure 1. Dr. Norm Johnson (The Ohio State University), Sara Hemly (The Ohio State University) and Elijah Talamas (Systematic Entomology Laboratory, ARS-USDA) helped immensely in the data management for this project through vSyslab. Morgan Rondonali (intern, 
Smithsonian Institution) and Elizabeth Roberts (Systematic Entomology Laboratory, ARS-USDA) carefully and efficiently databased most of the specimens used in this study. Smithsonian Institution interns Emily Fisher, Corrinne Cusick, Lauren McDevitt, and Matthew Johnson helped with the massive effort of mounting and labeling many of the specimens used in this study. Part of this study was supported by Comisión de Investigaciones Científicas de la Provincia de Buenos Aires (CIC) and Universidad Nacional de La Plata (UNLP). MLB was supported by the Systematic Entomology Laboratory, ARS-USDA. We also thank two anonymous reviewers for improving this manuscript. Mention of trade names or commercial products in this publication is solely for the purpose of providing specific information and does not imply recommendation or endorsement by the USDA. USDA is an equal opportunity provider and employer.

\section{Literature Cited}

Ashmead, W. H. 1894. Report upon the parasitic Hymenoptera of the Island of St. Vincent. Journal of the Linnean Society of London 25: 56-254.

Ashmead, W. H. 1896. Descriptions of new parasitic Hymenoptera. Transactions of the American Entomological Society 23: 179190.

Ashmead, W. H. 1900. Report upon the Aculeate Hymenoptera of the Islands of St. Vincent and Grenada, with additions to the parasitic Hymenoptera and a list of the described Hymenoptera of the West Indies. Transactions of the Entomological Society of London 1900: 206-367.

Ashmead, W. H. 1903a. Some new genera in the Cynipoidea. Proceedings of the Entomological Society of Washington 5: 221-223.

Ashmead, W. H. 1903b. Classification of the gall-wasps and the parasitic cynipoids, or the superfamily Cynipoidea. II. Psyche 10: 59-73.

Borgmeier, T. 1935. Sobre alguns Cynipideos parasiticos e cecidogenos do Brasil (Hymenoptera,
Cynipidae). Arquivos do Instituto de Biologia Vegetal 2: 97-124.

Buffington, M. L. 2002. A description of Aegeseucoela Buffington, new name, with taxonomic notes on the status of Gronotoma Förster. Proceedings of the Entomological Society of Washington 104: 589-601.

Buffington, M. L., S. Brady, S. Morita, and S. van Noort. 2012. Divergence estimates and early evolutionary history of Figitidae (Hymenoptera: Cynipoidea). Systematic Entomology 37: 287-304.

Buffington, M. L., R. Burks, and L. McNeil. 2005. Advanced techniques for imaging microhymenoptera. American Entomologist 51: 50-54.

Buffington, M. L. and M. Gates. 2009. Advanced imaging techniques II: using a compound microscope for photographing point-mount specimens. American Entomologist 54: 222 224.

Buffington, M. L., J. A. A. Nylander, and J. Heraty. 2007. The phylogeny and evolution of Figitidae (Hymenoptera: Cynipoidea). Cladistics 23: 1-29.

Buffington, M. L. 2009. Description, circumscription and phylogenetics of the new tribe Zaeucoilini (Hymenoptera: Figitidae: Eucoilinae), including a description of a new genus. Systematic Entomology 34:162-187.

Buffington, M.L. and S. J. Scheffer. 2008. North American species of Agrostocynips Díaz (Hymenoptera: Figitidae: Eucoilinae), parasitoids of Agromyzidae (Diptera): bionomics and taxonomy. Zootaxa 1817: 39-48.

Buffington, M. L. and S. van Noort. 2009. A revision of Anacharoides Cameron, 1904 (Hymenoptera, Figitidae) with a description of a new species. ZooKeys 20: 245-274.

Buffington, M. L., S. G. Brady, S. I. Morita, S. Van Noort. 2012. Divergence estimates and early evolutionary history of Figitidae ( $\mathrm{Hy}$ menoptera: Cynipoidea). Systematic Entomology 37: 287-304.

Buffington M. L., E. E. Perkovsky, and S. G. Brady. 2014. The Description of Rovnoeucoila tympanomorpha Buffington and Perkovsky, a new genus and species of fossil eucoiline, with observations on the asynchronous evolution of Diglyphosematini (Hymenoptera: Figitidae: Eucoilinae). Proceedings of the Entomological Society of Washington 116: 243-254.

Dalla Torre, K. W. and J. J. Kieffer. 1910. Cynipidae, Das Teirreich V. 24, 891 p. Verlag von R. Friedlander und Sohn, Berlin. 
De Santis, L. and N. Díaz. 1975. Himenópteros Chalcidoideos y Cynipoideos de la Isla Fernando de Noronha, Brazil (Hymenoptera: Cynipoidea and Chalcidoidea). Studia Entomologica 18: 193-200.

De Santis, L., N. B. Díaz, and I. Redolfii. 1976. La mosca del girasol (Diptera, Agromyzidae) y sus himenópteros parasitoides. Dusenia 9: 31-38.

Díaz, N. and F. E. Gallardo. 1997. Revisión sistemática de las especies del género Zaeucoila (Hymenoptera, Cynipoidea, Eucoilidae). Revista Nicaraguense de Entomología 39: 31-40.

Díaz, N. and F. E. Gallardo. 1998. Revision sistematica del genero Moneucoela (Hymenoptera: Figitidae). Revista de la Sociedad Entomológica Argentina 57: 111-113.

Díaz, N. and G. Valladares. 1979. Nota sobre Agrostocynips clavatus y los agromícidos hospedantes (Hymenoptera, Cynipoidea). Neotropica 25: 23-26.

Fontal-Cazalla, F. M., M. L. Buffington, G. Nordlander, J. Liljeblad, P. Ros-Farré, J. L. Nieves-Aldrey, J. Pujade-Villar, and F. Ronquist 2002. Phylogeny of the Eucoilinae (Hymenoptera: Cynipoidea: Figitidae). Cladistics 18: 154199.

Fontal, F. and J. L. Nieves-Aldrey., 2004. Estudio comparado de diversidad de eucoilinos paleárticos (El Ventorrillo, España) y neotropicales (P. N. Coiba, Panamá) (Hymenoptera, Cynipoidea, Figitidae, Eucoilinae). Boletín de la Sociedad Entomológica Aragonesa 35: 51-101.

Forshage, M., G. Nordlander, and M. L. Buffington. 2013. Eucoilinae of North America: a revised catalog of genera and described species. Proceedings of the Entomological Society of Washington 115: 225-255.

Gallardo, F. E. and N. B. Díaz. 1999. Revision sistematica de las especies del genero Lopheucoila Weld (Figitidae, Eucoilinae). Revista Nicaraguense de Entomología 47: 15-23.

Gallardo, F. E. and A. C. Dughetti. 2013. Zaeucoila unicarinata (Hymenoptera, Figitidae), parasitoide de Liryiomyza sp. (Diptera, Agromyzidae), plaga potencial en cultivos de cebolla del Valle Bonaerense del Río Colorado, Argentina. Acta Zoológica Lilloana, suplemento 57: 57-58.

Harris, R. A. 1979. A glossary of surface sculpturing. Occasional Papers in Entomology 28: $1-31$.
Kerr, P., E. Fisher, and M. L. Buffington. 2009. Dome lighting for insect imaging under a microscope. American Entomologist 54: 198-200.

Kieffer, J. J. 1907. Beschreibung neuer parasitischer Cynipiden aus Zentral- und Nord-Amerika. Entomologische Zeitschrift 21: 70-162.

Lorini, I. and L. A. Foerster. 1985. Flutuação populacional e parasitismo de Liriomyza sativae Blanchard, 1938 (Diptera Agromyzidae) na cultura do pepino (Cucumis sativus L.). Anais da Sociedade Entomologica do Brasil 14: 243-249.

Marchiori, C. H. and A. T. Oliveira. 2001. Primeiro relato de Zaeucoila incompleta (Kieffer) (Hymenoptera: Figitidae) como parasitóide de diptera no Brasil. Neotropical Entomology 30: 337-338.

Marchiori, C. H., M. H. O. Silva, and B. M. C Brito. 2004. Primeiro relato de Zaeucoila triangulifera Kieffer, 1907 (Hymenoptera: Figitidae: Eucoilinae) no Brasil. Biotemas 17: 225-229.

Nordlander, G. 1982. Systematics and phylogeny of an interrelated group of genera within the family Eucoilidae (Insecta: Hymenoptera, Cynipoidea). Doctoral dissertation. University of Stockholm, Sweden.

Rohwer, S. A. and M. M. Fagan. 1917. The typespecies of the genera of the Cynipoidea, or the gall wasps and the parasitic Cynipoids. Proceedings of the United States National Museum 53: 357-380.

Salvo, A. 2008. Parasitoides de minadores de hojas, pp. 31-51. In Claps L.E., G. Debandi and S. Roig-Juñent, eds. Biodiversidad de Artrópodos Argentinos Volúmen 2. Sociedad Entomológica Argentina, Mendoza.

Selegatto, A., N. Perioto, R. Lara, and N. Martinelli. 2004. Zaeucoila Ashmead, 1903 (Hymenoptera: Figitidae) em cultura de algodão no Estado de São Paulo, Brasil. In: Resumos XX Congresso Brasileiro de Entomologia, p. 624, Gramado, RS, Brasil.

Weld, L. H. 1921. Notes on certain genera of parasitic Cynipidae proposed by Ashmead with descriptions of genotypes. Proceedings of the United States National Museum 59: 433-451.

Weld, L. H. 1952. Cynipoidea (Hym.) 1905-1950. 351 p. Privately Printed, Ann Arbor, Michigan. 